\title{
REGULARIDADE DE CURVAS \\ MINIMIZANTES EM ESPAÇOS \\ SUB-RIEMANNIANOS HOMOGÊNEOS
}

\section{Daniel Victor Tausk}

\author{
DISSERTAÇÃO APRESENTADA AO \\ INSTITUTO DE MATEMÁTICA E \\ ESTATÍSTICA DA UNIVERSIDADE DE \\ SÃO PAULO PARA OBTENÇÃO DO GRAU \\ DE MESTRE EM MATEMÁTICA
}

Área de Concentração: Geometria

Orientador: Prof. Dr. Claudio Gorodski

Durante a elaboração desse trabalho o autor recebeu apoio financeiro da FAPESP.

- São Paulo, Junho de 1998 - 


\title{
REGULARIDADE DE CURVAS MINIMIZANTES EM ESPAÇOS SUB-RIEMANNIANOS HOMOGÊNEOS
}

\author{
Este exemplar corresponde à redação \\ final da dissertação de mestrado \\ devidamente corrigida e defendida \\ por Daniel Victor Tausk e aprovada \\ pela comissão julgadora.
}

São Paulo, 19 de junho de 1998

Banca examinadora:

- Prof. Dr. Claudio Gorodski (Orientador) - IME-USP

- Prof. Dr. José Antônio Verderesi - IME-USP

- Prof. Dr. Nikolai Goussevskii - UFMG 


\section{RESUMO}

Estudamos o problema da regularidade de curvas minimizantes do comprimento de arco em variedades sub-Riemannianas. Construímos uma família original de variedades sub-Riemannianas homogêneas onde conseguimos demonstrar que toda curva minimizante parametrizada por comprimento de arco é infinitamente diferenciável. Isso generaliza um resultado similar obtido por Montgomery para grupos de Lie compactos com distribuição invariante à esquerda ortogonal a um toro maximal. Fazemos uma exposição auto-contida da teoria básica das álgebras de Lie involutivas ortogonais, a qual é usada na construção das variedades sub-Riemannianas estudadas.

\section{ABSTRACT}

We study the problem of the regularity of arc length minimizing curves on subRiemannian manifolds. We construct an original family of examples of homogeneous subRiemannian manifolds in which we can prove that every minimizing curve parametrized by arc length is smooth. This generalizes a similar theorem proved by Montgomery for compact Lie groups with a left invariant distribution orthogonal to a maximal torus. We make a self-contained exposition of the basic theory of orthogonal involutive Lie algebras, which is used in the construction of the sub-Riemannian manifolds studied here. 


\section{Introdução.}

Geometria sub-Riemanniana é a geometria de distribuições não-integráveis de $k$-planos $\mathcal{D}$ em variedades diferenciáveis $M n$-dimensionais, $1<k<n$, onde $\mathcal{D}$ está equipada com uma métrica definida positiva $g$. Vide $[\mathrm{Kp}],[\mathrm{Mg}],[\mathrm{VG}],[\mathrm{FG}]$ para uma introdução e detalhes sobre o assunto. Note que quando $k=n$, nós recuperamos a geometria Riemanniana, mas no caso sub-Riemanniano aparecem fenômenos novos e interessantes como os descritos nas referências acima. Estamos especialmente interessados no fenômeno dos minimizantes anormais, como será explicado abaixo.

Em geometria Riemanniana, sabemos que toda curva que minimiza comprimento de arco (e é parametrizada por comprimento de arco) é uma geodésica. Além do mais, todo segmento suficientemente pequeno de uma geodésica minimiza comprimento de arco. Temos várias possíveis caracterizações para as geodésicas de uma variedade Riemanniana. Podemos defini-las como curvas que possuem campo tangente paralelo em relação à conexão de Levi-Civita, como soluções das equações de Euler-Lagrange associadas ao lagrangeano $L(q, \dot{q})=\frac{1}{2}\|\dot{q}\|^{2}$, como soluções das equações de Hamilton-Jacobi associadas ao hamiltoniano $H(q, p)=\frac{1}{2}\|p\|^{2}$ ou como pontos críticos do funcional energia $E(\gamma)=\frac{1}{2} \int_{a}^{b}\|\dot{\gamma}\|^{2}$ restrito ao espaço de curvas que ligam dois pontos dados.

Em geometria sub-Riemanniana seria desejável obter uma caracterização do mesmo tipo para curvas minimizantes. Ressaltamos que no caso sub-Riemanniano o comprimento de arco e a energia só podem ser definidos para curvas horizontais, i.e., curvas sempre tangentes à distribuição.

O lagrangeano $L(q, \dot{q})=\frac{1}{2}\|\dot{q}\|^{2}$ não é bem definido em todo o fibrado tangente $T M$, mas apenas em $\mathcal{D}$. Por outro lado, o hamiltoniano $H(q, p)=\frac{1}{2}\|p\|^{2}$ é bem definido em todo o fibrado cotangente $T M^{*}$ (temos uma aplicação canônica $T M^{*} \rightarrow \mathcal{D}^{*}$, dada por $p \mapsto p_{\mid \mathcal{D}}$ que nos permite estender aplicações definidas em $\mathcal{D}^{*}$ para $\left.T M^{*}\right)$. Podemos então considerar as soluções das equações de Hamilton-Jacobi, que denominaremos por extremais normais.

É de fato verdade que todo segmento suficientemente pequeno de um extremal normal minimiza comprimento de arco (vide [LS]). No caso Riemanniano, os extremais normais coincidem obviamente com as geodésicas. Em algumas classes de variedades subRiemannianas onde existem conexões canonicamente adaptadas à estrutura sub-Riemanniana (por exemplo, para variedades de contato. Vide [FG]), pode-se escrever a equação dos extremais normais em termos da conexão (não é verdade porém que extremais normais sejam as curvas com campo tangente paralelo).

Segue agora naturalmente a pergunta se toda curva minimizante (parametrizada por comprimento de arco) é um extremal normal. Uma resposta afirmativa para esse resultado chegou a ser enunciada em 1986 em [St], sugerindo-se uma demonstração baseada no princípio do máximo de Pontryagin. No entanto, desse princípio segue apenas que toda curva minimizante é um extremal normal ou então pertence a uma outra classe de curvas denominadas extremais anormais (definidas na seção 1).

A possibilidade da existência de minimizantes que não fossem normais já tinha sido sugerida em 1977 por B. Gaveau. Afirmações de que todos os minimizantes seriam normais foram no entanto novamente publicadas em $1988 \mathrm{em} \mathrm{[B]} \mathrm{e} \mathrm{em} 1990 \mathrm{em}$ [Hm].

A questão foi finalmente resolvida por R. Montgomery em 1991 em [Mg2], através de 
um exemplo explícito de uma curva minimizante que não é um extremal normal. O exemplo foi simplificado em 1992 por I. Kupka em [Kp2]. A demonstração de que minimizantes que não são normais existem sob condições bem gerais foi feita em [LS].

Pode-se também estudar a questão das curvas minimizantes em geometria sub-Riemanniana através de técnicas de cálculo variacional (em vez de técnicas de teoria do controle, como o princípio do máximo). Coloca-se no espaço das curvas horizontais uma estrutura de variedade de Banach, na qual procuramos os pontos críticos do funcional energia (restrito ao espaço das curvas que ligam dois pontos fixados). Tais pontos críticos coincidem de fato com os extremais normais. Ocorre no entanto, que o espaço das curvas horizontais que ligam dois pontos fixados em geral não é uma subvariedade de Banach do espaço das curvas horizontais. Os pontos em torno dos quais tal estrutura de subvariedade não existe está coberto pela classe dos extremais anormais (para uma analogia em dimensão finita, suponha que procuramos os mínimos de uma função diferenciável $f$ num cone. Consideramos então os pontos críticos de $f$. Devemos porém acrescentar o vértice do cone na lista de "candidatos" a mínimo, já que em torno do mesmo não temos uma estrutura de subvariedade de $\mathbb{R}^{3}$ e as técnicas de cálculo diferencial não se aplicam).

Como veremos no texto (exemplo 1.1), ocorre com freqüência que a "maioria" dos extremais anormais não possui nenhuma propriedade de minimização, i.e., temos condições necessárias de minimização que estão longe de ser suficientes. Outro fenômeno interessante, é que não existe uma equação diferencial cujas soluções sejam os extremais anormais (vide comentário que precede a observação 1.4).

Surge agora a questão da regularidade das curvas minimizantes. A descrição dos extremais normais em termos de soluções de equações diferenciais ordinárias deixa evidente o fato que os mesmos são de classe $C^{\infty}$. No entanto, é bastante fácil dar exemplos de extremais anormais que não são de classe $C^{\infty}$ (mesmo parametrizados por comprimento de arco). Aparece então a pergunta se todo extremal anormal minimizante é de classe $C^{\infty}$. Não são conhecidos contra-exemplos para essa afirmação e o problema geral continua em aberto. Alguns casos particulares foram resolvidos, por exemplo em [Mg3] (onde se consideram grupos de Lie compactos com distribuição invariante à esquerda ortogonal a um toro maximal).

O objetivo principal desse trabalho é o de descrever mais uma classe de variedades sub-Riemannianas onde conseguimos mostrar que toda curva minimizante (parametrizada por comprimento de arco) é de classe $C^{\infty}$. Esse é o conteúdo do teorema 4.4.

A técnica de demonstração (já usada em [Mg3]) baseia-se no seguinte. Considera-se um extremal anormal $\gamma$ da variedade $M$ em questão e mostra-se que o mesmo tem imagem dentro de uma subvariedade $N$. É fácil ver que $\gamma$ continua minimizante dentro de $N$. Se conseguirmos mostrar que $\gamma$ é um extremal normal dentro de $N$, seguirá que $\gamma$ é de classe $C^{\infty}$ (a técnica é bem ilustrada no exemplo 1.1 e no teorema 1.5).

No teorema 4.4 fazemos um processo indutivo. Se $\gamma$ não é um extremal normal de $N$ então $\gamma$ será necessariamente um extremal anormal em $N$ e conseguimos então uma subvariedade de $N$ que contém a imagem de $\gamma$. O processo continua até que encontremos uma subvariedade onde $\gamma$ é um extremal normal (e portanto é de classe $C^{\infty}$ ), ou diminuimos a dimensão da variedade ambiente até concluirmos que $\gamma$ é constante.

Ressaltamos que essa técnica nem sempre funciona. Sussmann em [Sm] mostra um 
exemplo de um extremal anormal que não é normal em nenhuma subvariedade. O exemplo consiste num extremal anormal que não é de classe $C^{\infty}$ em $\mathbb{R}^{3} \mathrm{com}$ uma distribuição de codimensão 1 (esse extremal anormal não é minimizante, no entanto. Como já foi explicado, não são conhecidos exemplos de curvas minimizantes que não são de classe $C^{\infty}$. Uma curva minimizante de classe $C^{\infty}$ é sempre normal em si própria, vista como variedade de dimensão 1). Em [GK] temos um exemplo de um grupo de Lie nilpotente (com distribuição invariante à esquerda) que admite uma curva minimizante anormal que não é normal em nenhum subgrupo (a classe de espaços homogêneos considerada no nosso texto é obtida por quociente de grupos de Lie semi-simples e portanto nunca nilpotentes). 


\section{Definições básicas.}

Começamos introduzindo o conceito de variedade sub-Riemanniana e a linguagem básica de curvas minimizantes e extremais. Ao longo do texto todos os objetos serão diferenciáveis, onde diferenciável significa de classe $C^{\infty}$, exceto quando for feita menção explícita em contrário (tipicamente, não suporemos a priori que curvas sejam diferenciáveis). Todas as variedades envolvidas serão Hausdorff com base enumerável (e reais, de dimensão finita).

Seja então $M$ uma variedade de dimensão $n$. Seja $\mathcal{D}$ uma distribuição em $M$ de posto $k$, i.e., $\mathcal{D}$ é um subfibrado vetorial do fibrado tangente $T M$ cujas fibras tem dimensão $k$. Mais explicitamente, $\mathcal{D} \subset T M$ é um subconjunto tal que para todo $m \in M$, a interseção $\mathcal{D}_{m}=\mathcal{D} \cap T_{m} M$ é um subespaço do espaço tangente $T_{m} M$ de dimensão $k$ e tal que existem campos vetorais $X^{1}, \ldots, X^{k}$ definidos numa vizinhança aberta $U$ de $m$, tais que os vetores $X_{x}^{1}, \ldots, X_{x}^{k}$ formam uma base de $\mathcal{D}_{x}$ para todo $x \in U$. Uma tal seqüência de campos vetoriais será chamada um referencial de $\mathcal{D}$. O número $n-k$ é chamado a codimensão de $\mathcal{D}$.

Uma métrica sub-Riemanniana em $(M, \mathcal{D})$ é um seção $g$ do fibrado $\mathcal{D}^{*} \otimes \mathcal{D}^{*}$ tal que para cada $m \in M, g_{m}$ é um produto interno (simétrico, definido positivo) em $\mathcal{D}_{m}$ (ou seja, $g$ é uma "escolha diferenciável de produto interno" nas fibras de $\mathcal{D}$ ). A diferenciabilidade de $g$ pode ser expressa mais explicitamente dizendo que para todo referencial $X_{1}, \ldots, X_{k}$ de $\mathcal{D}$, as funções $g\left(X_{i}, X_{j}\right)$ são diferenciáveis. Observe que quando $\mathcal{D}=T M$ obtemos simplesmente uma métrica Riemanniana em $M$. Chamamos à trinca $(M, \mathcal{D}, g)$ uma variedade sub-Riemanniana. O par $(\mathcal{D}, g)$ será também chamado uma estrutura sub-Riemanniana em $M$. Em geral, nos referiremos apenas à variedade sub-Riemanniana $M$, deixando a distribuição e a métrica subentendidas.

Observação 1.1. Em geral, geometria sub-Riemanniana torna-se mais interessante quando impomos condições de não integrabilidade à distribuição. Por exemplo, se $\mathcal{D}$ é integrável então uma variedade sub-Riemanniana reduz-se a uma folhação de variedades Riemannianas (pelo teorema de Frobenius). Em alguns textos, a definição de variedade sub-Riemanniana inclui condições mínimas de não integrabilidade, como a de que $\mathcal{D}$ seja geradora por colchetes. Denote por $\Gamma(T M)$ a álgebra de Lie (sobre $\mathbb{R}$ ) dos campos vetoriais em $M$ e por $\Gamma(\mathcal{D})$ o subespaço vetorial dos campos a valores em $\mathcal{D}$. Denote por $[\Gamma(\mathcal{D})]$ a subálgebra de Lie gerada por $\Gamma(\mathcal{D})$ em $\Gamma(T M)$. Dizemos que $\mathcal{D}$ é geradora por colchetes quando para todo $m \in M$, a imagem da aplicação $X \in[\Gamma(\mathcal{D})] \mapsto X(m)$ é todo o espaço tangente $T_{m} M$. Essa definição será motivada na observação 1.2, a seguir.

Passamos agora às definições de comprimento de arco e de curva minimizante. As curvas consideradas para esse fim serão sempre absolutamente contínuas. Recordamos que uma curva $\gamma:[a, b] \rightarrow \mathbb{R}^{n}$ é dita absolutamente contínua quando dado $\epsilon>0$ existe $\delta>0$ tal que se $\left(x_{1}, y_{1}\right), \ldots,\left(x_{r}, y_{r}\right)$ são intervalos abertos disjuntos contidos em $[a, b]$ com $\sum_{i=1}^{r} y_{i}-x_{i}<\delta$ então $\sum_{i=1}^{r}\left\|\gamma\left(y_{i}\right)-\gamma\left(x_{i}\right)\right\|<\epsilon$. Isso equivale a dizer que $\gamma$ é derivável quase sempre (i.e., o vetor tangente $\dot{\gamma}(t)$ existe para $t \in[a, b]$ fora de um conjunto de medida nula), $\dot{\gamma}:[a, b] \rightarrow \mathbb{R}^{n}$ é (Lebesgue) integrável e $\gamma(t)=\gamma(a)+\int_{a}^{t} \dot{\gamma}(s) d s$ para todo $t \in[a, b]$ (vide [Fe]). Mais geralmente, diremos que uma curva $\gamma:[a, b] \rightarrow M$ é absolutamente contínua quando suas representações locais em coordenadas o forem. 
Seja então $\gamma:[a, b] \rightarrow M$ uma curva absolutamente contínua numa variedade subRiemanniana $M$. Dizemos que $\gamma$ é horizontal quando $\dot{\gamma}(t) \in \mathcal{D}$ para quase todo $t \in[a, b]$ (quando $\gamma$ é $C^{1}$ então na verdade $\dot{\gamma}(t) \in \mathcal{D}$ para todo $t \in[a, b]$ ). Se $\gamma$ é horizontal definimos o comprimento de arco de $\gamma$ fazendo:

$$
L(\gamma)=\int_{a}^{b}\|\dot{\gamma}(s)\| d s,
$$

onde para $v \in \mathcal{D}_{m}$ escrevemos $\|v\|=g_{m}(v, v)^{1 / 2}$. Dizemos que $\gamma$ é parametrizada por comprimento de arco (pca) quando $\|\dot{\gamma}(t)\|=1$ para quase todo $t \in[a, b]$. Isso equivale a dizer que $L\left(\gamma_{\mid[a, t]}\right)=t-a$ para todo $t \in[a, b]$.

Observação 1.2. O teorema de Chow implica que quando $\mathcal{D}$ é geradora por colchetes e $M$ é conexa então quaisquer dois pontos de $M$ podem ser ligados por uma curva horizontal (que pode, na verdade, ser escolhida diferenciável por partes). Daí, como em geometria Riemanniana, podemos definir a distância entre dois pontos de $M$ como sendo o ínfimo dos comprimentos das curvas horizontais que os conectam. Não é difícil mostrar que obtemos então uma estrutura de espaço métrico em $M$. Na verdade, essa métrica induz a topologia de $M$ (vide [St]).

Dizemos que uma curva horizontal $\gamma:[a, b] \rightarrow M$ minimiza comprimento de arco quando para toda curva horizontal $\mu:[a, b] \rightarrow M \operatorname{com} \gamma(a)=\mu(a)$ e $\gamma(b)=\mu(b)$ tiver$\operatorname{mos} L(\gamma) \leq L(\mu)$. Nosso objetivo agora é o de enunciar condições necessárias para que uma curva minimize comprimento de arco.

Recordamos alguns fatos básicos sobre a geometria simplética do fibrado cotangente. Temos em $T M^{*}$ uma 1-forma canônica definida por:

$$
\theta_{p}(\lambda)=p\left(d \pi_{p}(\lambda)\right)
$$

para todo $p \in T M^{*}$ e $\lambda \in T_{p} T M^{*}$ (onde $\pi$ denota a projeção do fibrado cotangente). Definimos $\omega=-d \theta$. Temos que $\omega$ é uma forma simplética em $T M^{*}$, i.e., $d \omega=0$ e $\omega_{p}$ é uma 2-forma não degenerada em $T_{p} T M^{*}$ para cada $p \in T M^{*}$ (isso segue da representação em coordenadas locais que será vista abaixo). Diremos que $\omega$ é a forma simplética canônica do fibrado cotangente $T M^{*}$.

Seja $\varphi: U \rightarrow \tilde{U}$ uma carta em $M$, com $U \subset M$ e $\tilde{U} \subset \mathbb{R}^{n}$ abertos. Escrevemos $\varphi=$ $\left(q_{1}, \ldots, q_{n}\right)$. Obtemos então cartas $\bar{\varphi}: \pi^{-1}(U) \rightarrow \tilde{U} \times \mathbb{R}^{n}$ e $\bar{\varphi}^{*}: \pi^{-1}(U) \rightarrow \tilde{U} \times \mathbb{R}^{n *}$ em $T M$ e $T M^{*}$ respectivamente, definidas por $\bar{\varphi}(v)=\left(\varphi(m), d \varphi_{m}(v)\right)$ e $\bar{\varphi}^{*}(p)=\left(\varphi(m), d \varphi_{m}^{*}{ }^{-1}(p)\right)$, para $v \in T_{m} M, p \in T_{m} M^{*}$ e $m \in U$. Escrevemos $\bar{\varphi}=\left(q_{1}, \ldots, q_{n}, \dot{q}_{1}, \ldots, \dot{q}_{n}\right)$ e $\bar{\varphi}^{*}=$ $\left(q_{1}, \ldots, q_{n}, p_{1}, \ldots, p_{n}\right)$.

Obtemos então representações em coordenadas para $\theta$ e $\omega$ :

$$
\theta=\sum_{i=1}^{n} p_{i} d q_{i}, \quad \omega=\sum_{i=1}^{n} d q_{i} \wedge d p_{i} .
$$

Se $H: T M^{*} \rightarrow \mathbb{R}$ é uma função (chamada às vezes uma função hamiltoniana) então existe um único campo vetorial $\vec{H}$ em $T M^{*}$ tal que $d H_{p}(\lambda)=\omega_{p}\left(\vec{H}_{p}, \lambda\right)$ para $p \in T M^{*}$ e 
$\lambda \in T_{p} T M^{*}$. As projeções em $M$ das curvas integrais de $\vec{H}$ são chamadas soluções do hamiltoniano $H$ ou soluções das equações de Hamilton-Jacobi. Em coordenadas obtemos a forma clássica:

$$
\left\{\begin{array}{l}
\frac{d q}{d t}=\frac{\partial H}{\partial p} \\
\frac{d p}{d t}=-\frac{\partial H}{\partial q}
\end{array}\right.
$$

Seja agora $M$ uma variedade sub-Riemanniana. O produto interno $g_{m}$ em $\mathcal{D}_{m}$ induz um isomorfismo $v \mapsto g_{m}(v, \cdot)$ entre $\mathcal{D}_{m}$ e $\mathcal{D}_{m}^{*}$. Obtemos então um produto interno $g_{m}^{*}$ em $\mathcal{D}_{m}^{*}$. Definimos uma função hamiltoniana $H: T M^{*} \rightarrow \mathbb{R}$ fazendo:

$$
H(p)=\frac{1}{2} g_{m}^{*}\left(p_{\mid \mathcal{D}_{m}}, p_{\mid \mathcal{D}_{m}}\right),
$$

para $p \in T_{m} M^{*}$. As soluções das equações de Hamilton-Jacobi são chamadas os extremais normais de $M$. Note que é claro que extremais normais são sempre diferenciáveis.

Seja $\mathcal{D}^{0} \subset T M^{*}$ o anulador de $\mathcal{D}$ em $T M$, i.e., $\mathcal{D}^{0}$ é o subfibrado vetorial de $T M^{*}$ cuja fibra sobre o ponto $m \in M$ é o anulador de $D_{m}$ em $T_{m} M$. Temos que $\mathcal{D}^{0}$ é em particular uma subvariedade de $T M^{*}$ e podemos considerar a restrição de $\omega$ a $\mathcal{D}^{0}$. Essa restrição pode em geral se tornar degenerada e temos então para cada $p \in D^{0}$ um núcleo $\operatorname{Ker}\left(\omega_{\mid \mathcal{D}^{0}}\right)(p)$ formado pelos vetores $\lambda \in T_{p} \mathcal{D}^{0}$ tais que $\omega_{p}(\lambda, \mu)=0$ para todo $\mu \in T_{p} \mathcal{D}^{0}$.

Dizemos que uma curva absolutamente contínua $\eta:[a, b] \rightarrow T M^{*}$ é uma característica de $\mathcal{D}$ se $\eta([a, b]) \subset \mathcal{D}^{0}$ e $\dot{\eta}(t) \in \operatorname{Ker}\left(\omega_{\mid \mathcal{D}^{0}}\right)(\eta(t))$ para quase todo $t \in[a, b]$. Dizemos que uma curva $\gamma:[a, b] \rightarrow M$ é um extremal anormal se existe uma característica $\eta:[a, b] \rightarrow T M^{*}$ de $\mathcal{D}$ que nunca se anula e que se projeta sobre $\gamma$, i.e., $\gamma=\pi \circ \eta$.

Observe que, ao contrário da definição de extremal normal, a definição de extremal anormal não envolve a métrica $g$, mas apenas a distribuição $\mathcal{D}$.

Dizemos que $\gamma:[a, b] \rightarrow M$ é um extremal se for um extremal normal ou um extremal anormal (observamos que as duas possibilidades não são mutuamente exclusivas). $\mathrm{O}$ teorema abaixo motiva as definições acima. Uma demonstração pode ser encontrada em [LS].

Teorema 1.1. Seja $M$ uma variedade sub-Riemanniana e seja $\gamma:[a, b] \rightarrow M$ uma curva pca que minimiza comprimento de arco. Então $\gamma$ é um extremal.

Vamos olhar mais de perto o núcleo de $\omega_{\mid \mathcal{D}^{0}}$, utilizando uma trivialização de $\mathcal{D}^{0}$. Na descrição desse núcleo será útil considerarmos a forma de Levi, que será definida abaixo.

Fixada uma distribuição $\mathcal{D}$ em $M$, dizemos que um campo vetorial $X$ num aberto de $M$ é horizontal quando $X$ toma valores em $\mathcal{D}$. Se $X, Y$ são campos horizontais numa vizinhança de $m \in M$ então o colchete $[X, Y]_{m}$ módulo $\mathcal{D}_{m}$ depende apenas dos valores de $X$ e $Y$ no ponto $m$. Mais precisamente, se $X^{\prime}, Y^{\prime}$ são também campos horizontais numa vizinhança de $m$ com $X_{m}=X_{m}^{\prime}$ e $Y_{m}=Y_{m}^{\prime}$ então $[X, Y]_{m}-\left[X^{\prime}, Y^{\prime}\right]_{m} \in \mathcal{D}_{m}$. De fato, se $\sigma$ é uma 1-forma numa vizinhança de $m$ que anula $\mathcal{D}$ então aplicamos a fórmula de Cartan:

$$
d \sigma(X, Y)=X(\sigma(Y))-Y(\sigma(X))-\sigma([X, Y]) \Rightarrow d \sigma_{m}\left(X_{m}, Y_{m}\right)=-\sigma_{m}\left([X, Y]_{m}\right) .
$$

Aplicando (1.2) para os campos $X^{\prime}, Y^{\prime}$ vemos que:

$$
\sigma_{m}\left([X, Y]_{m}\right)=\sigma_{m}\left(\left[X^{\prime}, Y^{\prime}\right]_{m}\right),
$$


onde $\sigma_{m}$ é um elemento qualquer do anulador de $\mathcal{D}_{m}$ em $T_{m} M$. O resultado segue.

Podemos então para cada $m \in M$ definir um operador bilinear:

$$
\mathcal{L}_{m}: \mathcal{D}_{m} \times \mathcal{D}_{m} \rightarrow T_{m} M / \mathcal{D}_{m}
$$

fazendo $\mathcal{L}_{m}(v, w)=[X, Y]_{m}+\mathcal{D}_{m}$, onde $X, Y$ são campos horizontais quaisquer com $X_{m}=v, Y_{m}=w$ e para $u \in T_{m} M$ denotamos por $u+\mathcal{D}_{m}$ a sua classe no quociente $T_{m} M / \mathcal{D}_{m}$. Dizemos que $\mathcal{L}$ é a forma de Levi de $\mathcal{D}$. Novamente, se $\sigma$ é uma 1-forma numa vizinhança de $m$ que anula $\mathcal{D}$ então $\sigma_{m}$ passa ao quociente e define um funcional $\bar{\sigma}_{m} \in\left(T_{m} M / \mathcal{D}_{m}\right)^{*}$. A identidade (1.2) nos dá então:

$$
d \sigma_{m}(v, w)=-\bar{\sigma}_{m} \circ \mathcal{L}_{m}(v, w) .
$$

Observação 1.3. Note que a distribuição $\mathcal{D}$ é integrável se e somente se $\mathcal{L}_{m}=0$ para todo $m \in M$. A forma de Levi pode ser usada para descrever condições de não integrabilidade de $\mathcal{D}$, como será visto em exemplos abaixo.

Considere agora 1-formas $\sigma^{1}, \ldots, \sigma^{n-k}$ num aberto $U \subset M$ que formam uma base do anulador de $\mathcal{D}$ em cada ponto (i.e., um referencial de $\mathcal{D}^{0}$ ). Defina uma aplicação:

$$
\begin{gathered}
f: U \times \mathbb{R}^{n-k} \rightarrow T M^{*} \\
(m, c) \mapsto \sum_{i=1}^{n-k} c^{i} \sigma_{m}^{i} .
\end{gathered}
$$

Temos que $f$ é um difeomorfismo sobre a restrição de $\mathcal{D}^{0}$ a $U$ (na verdade, $f$ é uma trivialização de $\mathcal{D}^{0}$ sobre $U$ ). Vamos calcular as formas $f^{*} \theta$ e $f^{*} \omega$ e depois o núcleo de $f^{*} \omega$ (que corresponde pela diferencial de $f$ ao núcleo de $\omega_{\mid \mathcal{D}^{0}}$ ).

Temos, para $m \in U, v \in T_{m} M, c, h \in \mathbb{R}^{n-k}$ :

$$
\left(f^{*} \theta\right)_{(m, c)}(v, h)=\theta_{f(m, c)} \circ d f_{(m, c)}(v, h)=f(m, c) \circ d \pi_{f(m, c)} \circ d f_{(m, c)}(v, h),
$$

onde utilizamos apenas a definição de $\theta$ dada pela equação (1.1). Observando que $\pi \circ f$ é simplesmente a primeira projeção $\pi_{1}$ do produto $U \times \mathbb{R}^{n-k}$ e aplicando a regra da cadeia vem:

$$
\left(f^{*} \theta\right)_{(m, c)}(v, h)=\sum_{i=1}^{n-k} c^{i} \sigma_{m}^{i}(v) .
$$

Denotando por $\pi_{2}^{i}$ a projeção $(m, c) \mapsto c^{i}$ em $U \times \mathbb{R}^{n-k}$ para $i=1, \ldots, n-k$ obtemos:

$$
f^{*} \theta=\sum_{i=1}^{n-k} \pi_{2}^{i} \pi_{1}^{*} \sigma^{i} \quad \text { e } \quad f^{*} \omega=\sum_{i=1}^{n-k}-d \pi_{2}^{i} \wedge \pi_{1}^{*} \sigma^{i}-\pi_{2}^{i} \pi_{1}^{*} d \sigma^{i} .
$$

Temos finalmente, para $m \in M, v_{1}, v_{2} \in T_{m} M, c, h_{1}, h_{2} \in \mathbb{R}^{n-k}$ :

$$
\left(f^{*} \omega\right)_{(m, c)}\left(\left(v_{1}, h_{1}\right),\left(v_{2}, h_{2}\right)\right)=\sum_{i=1}^{n-k}-h_{1}^{i} \sigma_{m}^{i}\left(v_{2}\right)+h_{2}^{i} \sigma_{m}^{i}\left(v_{1}\right)-c^{i} d \sigma_{m}^{i}\left(v_{1}, v_{2}\right)
$$


Queremos determinar os pares $\left(v_{1}, h_{1}\right)$ que anulam a expressão (1.5) para valores arbitrários de $\left(v_{2}, h_{2}\right)$. Fazendo $v_{2}=0$ e deixando $h_{2} \in \mathbb{R}^{n-k}$ arbitrário vemos que necessariamente $v_{1} \in \mathcal{D}_{m}$. Daí o segundo termo dentro do somatório se anula. Obtemos que $(v, h) \in T_{m} M \times \mathbb{R}^{n-k}$ pertence ao núcleo de $f^{*} \omega$ no ponto $(m, c)$ se e somente se $v \in \mathcal{D}_{m}$ e:

$$
\sum_{i=1}^{n-k} h^{i} \sigma_{m}^{i}=-\sum_{i=1}^{n-k} c^{i} d \sigma_{m}^{i}(v, \cdot) .
$$

Escreva $\sigma=f(m, c) \in \mathcal{D}_{m}^{0}$. Daí $\sigma$ passa ao quociente e define um elemento $\vec{\sigma}$ em $\left(T_{m} M / \mathcal{D}_{m}\right)^{*}$. Utilizando a identidade (1.3) para as 1 -formas $\sigma^{i}$, vemos que a restrição a $\mathcal{D}_{m}$ da 1 -forma que aparece do lado direito da igualdade em (1.6) é simplesmente $\bar{\sigma} \circ \mathcal{L}_{m}(v, \cdot)$. Note que a 1-forma que aparece do lado esquerdo da igualdade em (1.6) sempre anula $\mathcal{D}_{m}$.

Fixado $v \in \mathcal{D}_{m}$ temos duas possibilidades. Se $\bar{\sigma} \circ \mathcal{L}_{m}(v, \cdot)$ anula $\mathcal{D}_{m}$ então existe um único $h \in \mathbb{R}^{n-k}$ que satisfaz (1.6). Caso contrário, tal $h$ não existe. Podemos visualizar o núcleo de $f^{*} \omega$ no ponto $(m, c)$ como o gráfico de um operador linear $v \mapsto h$, definido para $v$ pertencente ao núcleo da forma bilinear $\bar{\sigma} \circ \mathcal{L}_{m}$ em $\mathcal{D}_{m}$. Concluímos então o seguinte resultado.

Lema 1.2. Seja $M$ uma variedade e seja $\mathcal{D}$ uma distribuição em $M$. Seja $\omega$ a forma simplética canônica de $T M^{*}$ e seja $\sigma \in \mathcal{D}_{m}^{0}$. O núcleo de $\omega_{\mid \mathcal{D}^{0}}$ no ponto $\sigma$ se projeta isomorficamente por $d \pi_{\sigma}$ sobre o núcleo da forma bilinear $\bar{\sigma} \circ \mathcal{L}_{m}$ em $\mathcal{D}_{m}$ (onde definimos $\bar{\sigma} \in\left(T_{m} M / \mathcal{D}_{m}\right)^{*}$ a partir de $\sigma$ por passagem ao quociente).

Demonstração. O resultado segue dos cálculos que precedem o lema (observe que quando identificamos $\mathcal{D}_{\mid U}^{0} \operatorname{com} U \times \mathbb{R}^{n-k}$ pelo difeomorfismo $f$ em (1.4) então a projeção $\pi$ corresponde à primeira projeção $\pi_{1}$ do produto $\left.U \times \mathbb{R}^{n-k}\right)$.

A identidade (1.6) nos permite escrever uma equação para as características de $\mathcal{D}$. Seja $\eta:[a, b] \rightarrow \mathcal{D}^{0}$ uma curva absolutamente contínua e escreva $\gamma=\pi \circ \eta$. Suponha que $\gamma([a, b]) \subset U$. Podemos então escrever $\eta=f \circ(\gamma, c)$, onde $c:[a, b] \rightarrow \mathbb{R}^{n-k}$ é uma função absolutamente contínua e $f$ é o difeomorfismo definido em (1.4). Observe que temos simplesmente $\eta(t)=\sum_{i=1}^{n-k} c^{i}(t) \sigma_{\gamma(t)}^{i}$. Segue que $\eta$ é uma característica de $\mathcal{D}$ se e somente se $\gamma$ é horizontal e:

$$
\sum_{i=1}^{n-k} \dot{c}^{i}(t) \sigma_{\gamma(t)}^{i}=-\sum_{i=1}^{n-k} c^{i}(t) d \sigma_{\gamma(t)}^{i}(\dot{\gamma}(t), \cdot),
$$

para quase todo $t \in[a, b]$.

Suponha agora que temos um referencial $\sigma^{1}, \ldots, \sigma^{n}$ de $T M^{*}$ que completa o referencial $\sigma^{1}, \ldots, \sigma^{n-k}$ de $\mathcal{D}^{0}$. Daí, escrevemos para quase todo $t \in[a, b]$ :

$$
d \sigma_{\gamma(t)}^{i}(\dot{\gamma}(t), \cdot)=\sum_{j=1}^{n} \alpha_{j}^{i}(t) \sigma_{\gamma(t)}^{j}, \quad i=1,2, \ldots, n
$$

onde cada $\alpha_{j}^{i}:[a, b] \rightarrow \mathbb{R}$ é uma função (Lebesgue) integrável. Agora qualquer curva absolutamente contínua $\eta:[a, b] \rightarrow T M^{*}(\operatorname{com} \pi \circ \eta=\gamma)$ se escreve como $\eta(t)=\sum_{i=1}^{n} c^{i}(t) \sigma_{\gamma(t)}^{i}$, 
onde cada $c^{i}:[a, b] \rightarrow \mathbb{R}$ é uma função absolutamente contínua. Supondo $\gamma$ horizontal, segue de (1.7) que $\eta$ é uma característica de $\mathcal{D}$ se e somente se as funções $c^{i}$ formam uma solução do sistema de equações diferenciais ordinárias lineares homogêneas:

$$
\dot{c}^{j}(t)=-\sum_{i=1}^{n} c^{i}(t) \alpha_{j}^{i}(t), \quad j=1,2, \ldots, n,
$$

e também satisfazem o vínculo (algébrico) $c^{j}=0, j=n-k+1, \ldots, n$.

Observe que os extremais normais são simplesmente soluções de equações diferenciais ordinárias (não lineares), como as geodésicas de uma variedade Riemanniana. Os extremais anormais também estão associados a uma equação diferencial, como foi visto acima, mas a situação é diferente. Temos que, fixada uma curva horizontal $\gamma$ (a qual queremos verificar se é ou não um extremal anormal) então consideramos a equação diferencial ordinária linear (1.8) e verificamos se a mesma admite uma solução não nula que satisfaz um vínculo algébrico.

Observação 1.4. Sabemos que uma solução do sistema (1.8) ou é identicamente nula ou nunca se anula. Segue que poderíamos alternativamente ter definido que uma curva absolutamente contínua $\gamma:[a, b] \rightarrow M$ é um extremal anormal quando existe uma característica $\eta:[a, b] \rightarrow T M^{*}$ de $\mathcal{D}$ não identicamente nula tal que $\pi \circ \eta=\gamma$. Segue dos cálculos acima que uma tal característica na verdade nunca se anula.

Vamos agora utilizar o lema 1.2 para estudar o que ocorre com a projeção (por $d \pi_{\sigma}$ ) do núcleo de $\omega_{\mid \mathcal{D}^{0}}$ no ponto $\sigma$, quando fazemos $\sigma$ percorrer a fibra $\mathcal{D}_{m}^{0}$. Quando $\sigma=0$ vemos que essa projeção é toda a distribuição $\mathcal{D}_{m}$. Vamos nos ater então ao caso $\sigma \neq 0$. Em geral essa projeção depende de $\sigma$. Porém, quando $\mathcal{D}$ tem codimensão 1 temos que a fibra $\mathcal{D}_{m}^{0}$ é unidimensional e portanto essa projeção coincide sempre com o núcleo da forma de Levi, ou seja:

$$
\operatorname{Ker} \mathcal{L}_{m}=\left\{v \in \mathcal{D}_{m}: \mathcal{L}_{m}(v, w)=0, \forall w \in \mathcal{D}_{m}\right\}
$$

Note que nesse caso $T_{m} M / \mathcal{D}_{m} \cong \mathbb{R}$ e $\mathcal{L}_{m}$ é essencialmente uma forma bilinear (a valores reais) em $\mathcal{D}_{m}$. A constância da projeção do núcleo de $\omega_{\mid \mathcal{D}^{0}}$ quando variamos $\sigma$ dentro de uma fibra nos permite descrever os extremais anormais no caso de codimensão 1 sem recorrer a levantamentos para o fibrado cotangente. Temos o seguinte teorema.

Teorema 1.3. Seja $M$ uma variedade sub-Riemanniana cuja distribuição $\mathcal{D}$ tem codimensão 1. Então uma curva absolutamente contínua $\gamma:[a, b] \rightarrow M$ é um extremal anormal se e somente se $\dot{\gamma}(t) \in \operatorname{Ker} \mathcal{L}_{\gamma(t)}$ para quase todo $t \in[a, b]$.

Demonstração. Se $\gamma$ é um extremal anormal então temos uma característica $\eta:[a, b] \rightarrow \mathcal{D}^{0}$ que nunca se anula e tal que $\pi \circ \eta=\gamma$. Daí, para quase todo $t \in[a, b], \dot{\gamma}(t)=d \pi_{\eta(t)} \dot{\eta}(t)$ pertence à projeção (por $d \pi_{\eta(t)}$ ) do núcleo de $\omega_{\mid \mathcal{D}^{0}}$ no ponto $\eta(t) \neq 0$. Pelo que foi observado antes do teorema isso implica $\dot{\gamma}(t) \in \operatorname{Ker} \mathcal{L}_{\gamma(t)}$.

Supondo agora que $\dot{\gamma}(t) \in \operatorname{Ker} \mathcal{L}_{\gamma(t)}$ para quase todo $t \in[a, b]$, devemos construir uma característica $\eta:[a, b] \rightarrow \mathcal{D}^{0}$ que nunca se anula, tal que $\pi \circ \eta=\gamma$. Supondo que a imagem de $\gamma$ está contida num aberto $U \subset M$ sobre o qual $\mathcal{D}^{0}$ é trivial (i.e., sobre o qual existe uma 1-forma $\sigma$ cujo núcleo é $\mathcal{D}$ em cada ponto), mostraremos que $\eta$ fica unicamente 
determinada quando prescrevemos seu valor $\eta(t) \in \mathcal{D}_{\gamma(t)}^{0}, \eta(t) \neq 0$, para algum $t \in[a, b]$. Isso resolve o caso geral (basta dividir a curva $\gamma$ em pedaços e concatenar os levantamentos $\eta$ feitos com condições iniciais apropriadas).

Seja então $\sigma$ uma 1-forma num aberto $U \subset M$, cujo núcleo é $\mathcal{D}$ em cada ponto (i.e., $\sigma$ é um referencial de $\left.\mathcal{D}^{0}\right)$. Suponha que $\gamma([a, b]) \subset U$. Escrevemos as curvas absolutamente contínuas $\eta:[a, b] \rightarrow \mathcal{D}^{0}$ tais que $\pi \circ \eta=\gamma$ na forma $\eta(t)=c(t) \sigma_{\gamma(t)}$, para uma função absolutamente contínua $c:[a, b] \rightarrow \mathbb{R}$. Como $\gamma$ é horizontal, segue da equação (1.7) que $\eta$ é uma característica de $\mathcal{D}$ se e somente se:

$$
\dot{c}(t) \sigma_{\gamma(t)}=-c(t) d \sigma_{\gamma(t)}(\dot{\gamma}(t), \cdot),
$$

para quase todo $t \in[a, b]$. Como $\dot{\gamma}(t) \in \operatorname{Ker} \mathcal{L}_{\gamma(t)}$ segue da identidade (1.3) que a 1-forma $d \sigma_{\gamma(t)}(\dot{\gamma}(t), \cdot)$ anula $\mathcal{D}_{\gamma(t)}$ e portanto é da forma $\alpha(t) \sigma_{\gamma(t)}$. Fica definida então uma função integrável $\alpha:[a, b] \rightarrow \mathbb{R}$. Temos que $\eta$ é uma característica de $\mathcal{D}$ se e somente se:

$$
\dot{c}(t)=-\alpha(t) c(t)
$$

para quase todo $t \in[a, b]$. Mas a equação diferencial ordinária linear homogênea (1.3.1) tem solução única quando prescrevemos $c(t) \in \mathbb{R}$ para algum $t \in[a, b]$ e também $c$ nunca se anula se $c(t) \neq 0$.

Dizemos que uma distribuição $\mathcal{D}$ numa variedade $M$ é de contato quando tiver codimensão 1 e sua forma de Levi for não degenerada em cada ponto, i.e., $\operatorname{Ker} \mathcal{L}_{m}=0$ para todo $m \in M$. Segue diretamente do teorema acima que se $\mathcal{D}$ é de contato então todo extremal anormal é constante. Em particular, segue do teorema 1.1 que toda curva minimizante pca é um extremal normal (não é difícil ver que curvas constantes são sempre extremais normais também). Descrevemos agora uma condição mais fraca sob a qual concluímos que extremais anormais são sempre constantes.

Dizemos que $\mathcal{D}$ é fortemente geradora por colchetes quando dado $m \in M$ e um campo horizontal $X \operatorname{com} X(m) \neq 0$, obtemos:

$$
T_{m} M=\mathcal{D}_{m}+\left\{[X, Y]_{m}: Y \text { campo horizontal }\right\} .
$$

Isso equivale a dizer que dados $m \in M$ e $v \in \mathcal{D}_{m}, v \neq 0$, o operador linear $\mathcal{L}_{m}(v, \cdot)$ é sobrejetor. Quando $\mathcal{D}$ tem codimensão 1 essa condição equivale à condição de contato. Temos o seguinte lema.

Lema 1.4. Uma distribuição $\mathcal{D}$ é fortemente geradora por colchetes se e somente se $\omega_{\mid \mathcal{D}^{0}}$ é não degenerada (e portanto simplética) fora da seção nula de $\mathcal{D}^{0}$.

Demonstração. Pelo lema 1.2 , vemos que $\omega_{\mid \mathcal{D}^{0}}$ é não degenerada fora da seção nula de $\mathcal{D}^{0}$ se e somente se para todo $m \in M$ e todo $\sigma \in \mathcal{D}_{m}^{0}, \sigma \neq 0$, tivermos $\operatorname{Ker}\left(\bar{\sigma} \circ \mathcal{L}_{m}\right)=0$. Mas $\bar{\sigma}$ é um funcional não nulo arbitrário em $T_{m} M / \mathcal{D}_{m}$. Daí segue facilmente o resultado.

Corolário. Se $M$ é uma variedade sub-Riemanniana com distribuição fortemente geradora por colchetes então todo extremal anormal é constante. Em particular, toda curva minimizante pca é diferenciável.

Demonstração. Segue diretamente do lema e do teorema 1.1. 
Exemplo 1.1. O teorema 1.3 nos permite computar facilmente em exemplos concretos os extremais anormais de uma variedade sub-Riemanniana com distribuição de codimensão 1. Considere então $M=\mathbb{R}^{5}$ e denote por $x, y, z, t$ e $w$ as funções coordenadas de $\mathbb{R}^{5}$. Considere os campos vetoriais:

$$
X=\frac{\partial}{\partial x}, \quad Y=\frac{\partial}{\partial y}, \quad Z=\frac{\partial}{\partial z}, \quad T=x \frac{\partial}{\partial t}+\frac{\partial}{\partial w}
$$

É fácil ver que eles são linearmente independentes em cada ponto e portanto geram uma distribuição $\mathcal{D}$ em $M$ de posto 4. Considere uma métrica sub-Riemanniana $g$ arbitrária em $(M, \mathcal{D})$.

Observe que $[X, T]=\frac{\partial}{\partial t}$ e que $\frac{\partial}{\partial t}$ está sempre fora de $\mathcal{D}$. Isso mostra que a distribuição $\mathcal{D}$ é geradora por colchetes (vide observação 1.1). É fácil ver que os campos $Y$ e $Z$ ficam dentro do núcleo da forma de Levi e portanto formam uma base do mesmo (como $\mathcal{L}_{m}$ é antisimétrica, vemos que a dimensão de seu núcleo e a dimensão de $\mathcal{D}_{m}$ tem a mesma paridade. Mas sabemos no nosso caso que $\mathcal{L}_{m}$ nunca é nula e portanto seu núcleo deve ter dimensão 2).

Segue do teorema 1.3 que uma curva absolutamente contínua $\gamma:[a, b] \rightarrow \mathbb{R}^{5}$ é um extremal anormal se e somente se sua imagem está contida num plano afim paralelo ao gerado por $\frac{\partial}{\partial y}$ e $\frac{\partial}{\partial z}$, i.e., se e somente se as coordenadas $x, t$ e $w$ são constantes sobre a imagem de $\gamma$.

Esse exemplo mostra que, em muitos casos, a "maioria" dos extremais anormais não é minimizante (nem localmente). Vemos também que existem muitos extremais anormais (pca) que não são sequer de classe $C^{1}$. Observe no entanto que, no nosso exemplo, se algum extremal anormal pca for realmente minimizante então ele será uma geodésica (Riemanniana) do plano afim que o contém (considerado com a métrica Riemanniana obtida por restrição de $g$ ). Segue então que tal extremal será diferenciável. No teorema a seguir generalizamos esse raciocínio. Alguns fatos simples sobre distribuições integráveis e subvariedades integrais utilizados no teorema abaixo são explicados com mais detalhe no início da seção 4.

Observação 1.5. Seja $\mathcal{D} \subset T M$ um subconjunto tal que $\mathcal{D}_{m}=\mathcal{D} \cap T_{m} M$ é um subespaço de dimensão $k$ de $T_{m} M$ para todo $m \in M$. Suponha também que para todo $m \in M$ existem 1 -formas $\sigma^{1}, \ldots, \sigma^{r}$ numa vizinhança aberta $U$ de $m$ tais que $\sigma_{x}^{1}, \ldots, \sigma_{x}^{r}$ geram o anulador de $\mathcal{D}_{x}$ para todo $x \in U$ (mas não necessariamente formam uma base desse anulador). Então $\mathcal{D}$ é uma distribuição em $M$. De fato, fazendo uma reordenação, se necessário, podemos supor que $\sigma_{m}^{1}, \ldots, \sigma_{m}^{n-k}$ formam uma base do anulador de $\mathcal{D}_{m}$. Para $x$ numa vizinhança de $m$ temos então que $\sigma_{x}^{1}, \ldots, \sigma_{x}^{n-k}$ ainda são linearmente independentes e portanto ainda formam uma base do anulador de $\mathcal{D}_{x}$ (pois $\mathcal{D}_{x}$ tem dimensão $k$ ). Obtemos então um referencial de $\mathcal{D}^{0}$, o que mostra que $\mathcal{D}$ é uma distribuição.

Teorema 1.5. Seja $M$ uma variedade sub-Riemanniana cuja distribuição tem codimensão 1. Suponha que o núcleo $\operatorname{Ker} \mathcal{L}_{m}$ da forma de Levi tem dimensão constante (i.e., independente de $m \in M$ ). Então o mesmo define uma distribuição integrável Ker $\mathcal{L}$. Os extremais anormais de $M$ são precisamente as curvas absolutamente contínuas cuja imagem está 
contida numa subvariedade integral maximal de $\operatorname{Ker} \mathcal{L}$. Toda curva minimizante pca em $M$ será diferenciável.

Demonstração. Primeiro devemos mostrar que $\operatorname{Ker} \mathcal{L}$ é uma distribuição em $M$. Utilizamos a observação 1.5. Seja $m \in M$ e seja $\sigma$ uma 1-forma numa vizinhança de $m$ cujo núcleo é $\mathcal{D}$ em cada ponto. Seja também $X^{1}, \ldots, X^{n-1}$ um referencial de $\mathcal{D}$ numa vizinhaça de $m$. Pela identidade (1.3), as 1 -formas $\sigma$ e $d \sigma\left(X^{i}, \cdot\right), i=1,2, \ldots, n-1$ geram o anulador de $\operatorname{Ker} \mathcal{L}$ em cada ponto. Como $\operatorname{Ker} \mathcal{L}_{x}$ tem dimensão constante, o resultado segue.

Vamos mostrar agora que $\operatorname{Ker} \mathcal{L}$ é integrável. Observe primeiro que se um campo $X$ toma valores em $\operatorname{Ker} \mathcal{L}$ e $Y$ é horizontal (i.e., toma valores em $\mathcal{D}$ ) então $[X, Y]$ é horizontal. Suponha que $X$ e $Y$ ambos tomam valores em $\operatorname{Ker} \mathcal{L}$. Devemos mostrar que $[X, Y]$ toma valores em $\operatorname{Ker} \mathcal{L}$. Já sabemos que $[X, Y]$ é horizontal. Além do mais, se $Z$ é qualquer campo horizontal segue da identidade de Jacobi que:

$$
[[X, Y], Z]=[[X, Z], Y]+[X,[Y, Z]]
$$

e portanto $[[X, Y], Z]$ é horizontal. Logo $\mathcal{L}_{m}\left([X, Y]_{m}, Z_{m}\right)=0$, donde segue o resultado.

Pelo teorema 1.3, os extremais anormais de $M$ são exatamente as curvas absolutamente contínuas tangentes à distribuição integrável $\operatorname{Ker} \mathcal{L}$, ou equivalentemente, contidas numa subvariedade integral maximal de $\operatorname{Ker} \mathcal{L}$. Pelo teorema 1.1, toda curva minimizante pca em $M$ será um extremal normal (e portanto diferenciável) ou um extremal anormal. No segundo caso, é fácil ver que tal curva será minimizante pca numa subvariedade integral maximal de $\operatorname{Ker} \mathcal{L}$ (vista com a estrutura Riemanniana obtida por restrição de $g$ ) e portanto será uma geodésica dessa subvariedade.

O teorema acima nos fornece o primeiro exemplo não trivial de uma família de variedades sub-Riemannianas onde toda curva minimizante pca é diferenciável.

O teorema central da seção 3 (teorema 4.4) nos fornecerá uma outra família de exemplos desse tipo. A técnica de demonstração utilizada (i.e., mostrar que extremais anormais tem imagem dentro de certas subvariedades) é a mesma, embora a demonstração seja tecnicamente mais difícil. 


\section{Equações dos extremais anormais em espaços homogêneos.}

O objetivo desta seção é o de determinar equações para os extremais anormais de uma variedade sub-Riemanniana homogênea (ou, mais precisamente, com distribuição homogênea), utilizando a linguagem de grupos de Lie.

Sejam $M, M^{\prime}$ variedades munidas de distribuições $\mathcal{D}$ e $\mathcal{D}^{\prime}$ respectivamente. Dizemos que uma aplicação $f: M \rightarrow M^{\prime}$ preserva a distribuição quando $f$ for um difeomorfismo local e $d f_{m}$ se restringe a um isomorfismo de $\mathcal{D}_{m}$ em $\mathcal{D}_{f(m)}^{\prime}$ para todo $m \in M$. Quando $(M, \mathcal{D})$ e $\left(M^{\prime}, \mathcal{D}^{\prime}\right)$ estão munidas de métricas sub-Riemannianas $g$ e $g^{\prime}$ respectivamente, dizemos que $f$ é uma isometria local quando além do mais, $d f_{m}$ se restringe a uma isometria linear de $\mathcal{D}_{m}$ em $\mathcal{D}_{f(m)}^{\prime}$ para todo $m \in M$. Dizemos que $f$ é uma isometria (global) quando $f$ for uma isometria local bijetora. Quando existe uma isometria $f: M \rightarrow M^{\prime}$ dizemos $M$ e $M^{\prime}$ são isométricas.

Seja $M$ uma variedade munida de uma distribuição $\mathcal{D}$ (respectivamente, uma variedade sub-Riemanniana). Considere uma ação $G \times M \rightarrow M$ de um grupo de Lie $G$ em $M$. Dizemos que a distribuição $\mathcal{D}$ (respectivamente, a estrutura sub-Riemanniana) é $G$-invariante quando para todo $g \in G$ a aplicação $m \mapsto g \cdot m$ preserva a distribuição (respectivamente, é uma isometria). Dizemos também que a ação de $G$ preserva a distribuição (respectivamente, preserva a estrutura sub-Riemanniana). Dizemos que a distribuição (respectivamente, a variedade sub-Riemanniana) é homogênea quando existe uma ação transitiva de um grupo de Lie $G$ em $M$ que preserva a distribuição (respectivamente, a estrutura sub-Riemanniana).

Observação 2.1. No caso de geometria Riemanniana, sabe-se que o grupo de todas as isometrias de uma variedade é um grupo de Lie e portanto pode-se definir um espaço homogêneo simplesmente exigindo que dados $m, m^{\prime} \in M$ exista uma isometria $T$ com $T(m)=m^{\prime}$. No caso de geometria sub-Riemanniana tal resultado não é conhecido e a definição acima é mais apropriada.

Seja $M$ uma variedade sub-Riemanniana homogênea. Considere uma ação transitiva de um grupo de Lie $G$ em $M$ que preserva a estrutura sub-Riemanniana. Fixe um ponto $m_{0} \in M$. Seja $K=\left\{g \in G: g \cdot m_{0}=m_{0}\right\}$ o subgrupo de isotropia de $m_{0}$. Então $K$ é um subgrupo (de Lie) fechado de $G$ e portanto o conjunto quociente $G / K$ tem uma única estrutura de variedade diferenciável tal que a projeção $\pi: G \rightarrow G / K$ é uma submersão. Denotamos a classe (à esquerda) de um elemento $g \in G$ por $\pi(g)=g K$. Temos então um difeomorfismo canônico entre $G / K$ e $M$ dado por $g K \mapsto g \cdot m_{0}$ (vide [Wn]). Esse difeomorfismo define uma única estrutura sub-Riemanniana em $G / K$ que o torna uma isometria. A ação de $G$ em $M$ corresponde então à ação de $G$ em $G / K$ por translação à esquerda $g \cdot x K=g x K$. É claro que a estrutura sub-Riemanniana de $G / K$ também é $G$-invariante (em relação à ação de $G$ por translação à esquerda).

A partir de agora então não distinguiremos $M$ de $G / K$ e trabalharemos apenas com variedades sub-Riemannianas $(G / K, \mathcal{D}, g)$, onde $G$ é um grupo de Lie, $K$ é um subgrupo fechado de $G$ e a estrutura sub-Riemanniana é $G$-invariante (fica subentendido que a ação considerada de $G$ em $G / K$ é por translação à esquerda).

Introduzimos alguma notação. Seja $g \in G$. Denotamos por $l_{g}: G \rightarrow G$ a translação à esquerda $l_{g}(x)=g x$ e por $r_{g}: G \rightarrow G$ a translação à direita $r_{g}(x)=x g$. Denotamos por 
$\bar{l}_{g}: G / K \rightarrow G / K$ a translação à esquerda $\bar{l}_{g}(x K)=g x K$, i.e., a ação de $g$ em $G / K$ (note que não faz sentido definir $\bar{r}_{g}$ ). O automorfismo interno $x \mapsto g x g^{-1}$ será denotado por $\mathcal{I}_{g}$ e a representação adjunta de $G$ na sua álgebra de Lie g será denotada por $\operatorname{Ad}_{g}=d \mathcal{I}_{g}(1)$, onde $1 \in G$ denota o elemento neutro. Temos então um homomorfismo:

$$
\text { Ad: } G \rightarrow \operatorname{Aut}(\mathfrak{g}) \subset \mathrm{GL}(\mathfrak{g}),
$$

onde Aut(g) denota o grupo (de Lie) de automorfismos de $\mathfrak{g}$ e $\mathrm{GL}(\mathfrak{g})$ denota o grupo de isomorfismos lineares de $\mathfrak{g}$. Escreva $d \mathrm{Ad}_{1}=$ ad. Daí ad: $\mathfrak{g} \rightarrow \operatorname{Der}(\mathfrak{g}) \subset \mathcal{L}(\mathfrak{g})$ é a representação adjunta de $\mathfrak{g}$ em si mesma, onde $\operatorname{Der}(\mathfrak{g})$ denota a álgebra de Lie de derivações de $\mathfrak{g}$ e $\mathcal{L}(\mathfrak{g})$ denota a álgebra associativa de operadores lineares em $\mathfrak{g}$. Sabemos que $\operatorname{ad}_{X} Y=$ $[X, Y]$ (vide $[\mathrm{Wn}])$.

Por simplicidade, para $g \in G$ e $v \in T G$ escrevemos $g v$ em vez de $d l_{g}(v)$ e $v g$ em vez de $d r_{g}(v)$. Também para $v \in T(G / K)$ escrevemos $g v$ em vez de $d \bar{l}_{g}(v)$ (note que não faz sentido escrever $v g$ nesse caso).

Sabemos que $G$ possui uma estrutura de fibrado $K$-principal com base $G / K$ e projeção $\pi$ (vide [KN]). Essa linguagem não será importante para nosso texto, mas apenas definimos que uma seção local de $G$ relativa a $K$ é uma aplicação $s: U \rightarrow G$ definida num aberto $U \subset G / K$ tal que $\pi \circ s=\operatorname{Id}_{\mid U}$, i.e., $s(g K)$ é um elemento da classe $g K$ para todo $g K \in U$. A existência de seções locais (diferenciáveis) em torno de cada ponto de $G / K$ segue simplesmente do fato que $\pi$ é uma submersão.

Escreva $\bar{\pi}=d \pi_{1}: \mathfrak{g} \rightarrow T_{1 K}(G / K)$. Daí $\bar{\pi}$ é um operador linear sobrejetor cujo núcleo é a álgebra de Lie $\mathfrak{k}$ de $K$, donde identificamos $T_{1 K}(G / K)$ com o quociente $\mathfrak{g} / \mathfrak{k}$ de espaços vetoriais e daí $\bar{\pi}(X)=X+\mathfrak{k}$ para todo $X \in \mathfrak{g}$, onde $X+\mathfrak{k}$ denota a classe de $X$ no quociente $\mathfrak{g} / \mathfrak{k}$.

Dado $k \in K$ então $\mathcal{I}_{k}(K) \subset K$, donde $\operatorname{Ad}_{k}(\mathfrak{k}) \subset \mathfrak{k}$ e podemos por passagem ao quociente definir um operador linear:

$$
\overline{\operatorname{Ad}}_{k}: \mathfrak{g} / \mathfrak{k} \rightarrow \mathfrak{g} / \mathfrak{k},
$$

tal que $\overline{\operatorname{Ad}}_{k} \circ \bar{\pi}=\bar{\pi} \circ \operatorname{Ad}_{k}$. Temos que $\overline{\text { Ad }}$ define uma representação de $K$ em $\mathfrak{g} / \mathfrak{k}$. Afirmamos que essa coincide com a representação isotrópica de $K$, i.e., $\overline{\operatorname{Ad}}_{k}=d \bar{l}_{k}(1 K)$ para $k \in K$. De fato, isso segue se diferenciarmos a identidade $\pi \circ \mathcal{I}_{k}=\bar{l}_{k} \circ \pi$ no ponto $1 \in G$. Podemos também considerar $d \overline{\mathrm{Ad}}_{1}=\overline{\mathrm{ad}}$ e é fácil ver que $\overline{\mathrm{ad}}_{X}(Y+\mathfrak{k})=[X, Y]+\mathfrak{k}$ para $X \in \mathfrak{k}, Y \in \mathfrak{g}$, i.e., $\overline{\operatorname{ad}}_{X}$ é definida a partir de $\operatorname{ad}_{X}$ por passagem ao quociente também.

A transitividade da ação de $G$ em $G / K$ nos diz que uma estrutura sub-Riemanniana $G$-invariante em $G / K$ fica totalmente determinada pelo seu valor no ponto $1 K$. Além do mais, para $k \in K$ a diferencial de $\bar{l}_{k}$ no ponto $1 K$ se restringe a uma isometria de $\mathcal{D}_{1 K}$. Segue que as estruturas sub-Riemannianas $G$-invariantes em $G / K$ estão em correspondência biunívoca com os pares $(\mathfrak{d},\langle\rangle$,$) , onde \mathfrak{d} \subset \mathfrak{g} / \mathfrak{k}$ é um subespaço e $\langle$,$\rangle é um produto interno$ em $\mathfrak{O}$, ambos invariantes pela representação isotrópica de $K$ (a estrutura sub-Riemanniana pode então ser "espalhada" usando a ação de $G$. A diferenciabilidade da estrutura obtida se mostra usando seções locais).

Trabalhamos sempre então com uma trinca $(G / K, \mathfrak{o},\langle\rangle$,$) , onde \mathfrak{d} \subset \mathfrak{g} / \mathfrak{k}$ é um subespaço $\overline{\mathrm{Ad}}$-invariante e $\langle$,$\rangle é um produto interno \overline{\mathrm{Ad}}$-invariante em $\mathfrak{d}$. 
Observação 2.2. Note que estruturas invariantes pela ação $\overline{\mathrm{Ad}}$ do grupo $K$ são também invariantes pela ação ad da álgebra de Lie $\mathfrak{k}$ (e tais invariâncias são equivalentes quando $K$ é conexo). Em particular temos $[X, Y] \in \bar{\pi}^{-1}(\mathfrak{d})$ sempre que $X \in \mathfrak{k}$ e $Y \in \bar{\pi}^{-1}(\mathfrak{d})$, ou de maneira abreviada, $\left[\mathfrak{k}, \bar{\pi}^{-1}(\mathfrak{d})\right] \subset \bar{\pi}^{-1}(\mathfrak{d})$.

Como já foi observado na seção anterior, os extremais anormais de uma variedade subRiemanniana dependem apenas da distribuição e não da métrica, de modo que o produto interno em $\mathfrak{d}$ será irrelevante no que segue.

Nosso objetivo agora é o de obter fórmulas para as formas canônicas $\theta$ e $\omega$ em $T(G / K)^{*}$ em termos de uma seção local $s$. Fazemos uma pequena digressão para introduzir um pouco da linguagem de formas diferenciais a valores vetoriais.

Seja $M$ uma variedade e seja $V$ um espaço vetorial (real, de dimensão finita). Uma $r$-forma diferencial a valores em $V$ na variedade $M$ é uma seção $\omega$ do fibrado vetorial $\bigwedge_{r} T M^{*} \otimes V$. Temos então para cada $m \in M$ um operador $r$-linear alternado:

$$
\omega_{m}: \underbrace{T_{m} M \times \cdots \times T_{m} M}_{p \text { vezes }} \rightarrow V
$$

Destacamos que uma 0-forma a valores em $V$ é simplesmente uma função $\omega: M \rightarrow V$ a valores em $V$.

Se $f: N \rightarrow M$ é uma aplicação, podemos definir da maneira usual o pull-back $f^{*} \omega$, obtendo uma $r$-forma a valores em $V$ na variedade $N$ definida por:

$$
\left(f^{*} \omega\right)_{n}\left(v_{1}, \ldots, v_{r}\right)=\omega_{f(n)}\left(d f_{n}\left(v_{1}\right), \ldots, d f_{n}\left(v_{r}\right)\right)
$$

para $n \in N$ e $v_{1}, \ldots, v_{r} \in T_{n} N$.

Escolhida uma base $\left(e_{1}, \ldots, e_{d}\right)$ em $V$ obtemos uma seqüência $\left(\omega^{1}, \ldots, \omega^{d}\right)$ de formas (a valores reais) definidas por:

$$
\omega_{m}\left(v_{1}, \ldots, v_{r}\right)=\sum_{i=1}^{d} \omega_{m}^{i}\left(v_{1}, \ldots, v_{r}\right) e_{i}
$$

para $m \in M$ e $v_{1}, \ldots, v_{r} \in T_{m} M$. Essas formas são chamadas as coordenadas de $\omega$ nessa base. Podemos então definir a diferencial exterior de $\omega$ como sendo a $r+1$-forma a valores em $V$ cujas coordenadas são $\left(d \omega^{1}, \ldots, d \omega^{d}\right)$. Segue da $\mathbb{R}$-linearidade da diferenciação exterior que $d \omega$ não depende da base escolhida.

Sejam $V_{1}, V_{2}$ e $W$ espaços vetoriais (reais, de dimensão finita) e seja $p: V_{1} \times V_{2} \rightarrow W$ um operador bilinear. Se $\omega^{1}$ e $\omega^{2}$ são respectivamente uma $r$-forma a valores em $V_{1}$ e uma $s$-forma a valores em $V_{2}$ então podemos definir sua multiplicação exterior (em relação ao produto $p$ ) como sendo uma $r+s$-forma $\omega^{1} \wedge \omega^{2}$ a valores em $W$ definida por:

$$
\begin{aligned}
& \left(\omega^{1} \wedge \omega^{2}\right)_{m}\left(v_{1}, \ldots, v_{r}, v_{r+1}, \ldots, v_{r+s}\right)= \\
& \frac{1}{r ! s !} \sum_{\sigma \in S_{r+s}}(-1)^{\sigma} p\left(\omega_{m}^{1}\left(v_{\sigma(1)}, \ldots, v_{\sigma(r)}\right), \omega_{m}^{2}\left(v_{\sigma(r+1)}, \ldots, v_{\sigma(r+s)}\right)\right)
\end{aligned}
$$


para $m \in M$ e $v_{1}, \ldots, v_{r+s} \in T_{m} M$, onde $S_{r+s}$ representa o grupo de permutações de $\{1, \ldots, r+s\}$ e $(-1)^{\sigma}$ representa o sinal da permutação $\sigma$. Destacamos o caso particular em que ambas as formas $\omega^{1}, \omega^{2}$ tomam valores no mesmo espaço vetorial $V$ e o mesmo possui uma estrutura de álgebra associativa. Podemos então tomar $p: V \times V \rightarrow V$ como sendo a multiplicação de $V$. Note também que se $r=0$ então temos apenas:

$$
\left(\omega^{1} \wedge \omega^{2}\right)_{m}\left(v_{1}, \ldots, v_{s}\right)=p\left(\omega_{m}^{1}, \omega_{m}^{2}\left(v_{1}, \ldots, v_{s}\right)\right) .
$$

Se $T: V \rightarrow W$ é um operador linear e $\omega$ é uma $r$-forma em $M$ a valores em $V$ então podemos definir uma $r$-forma $T$ o $\omega$ em $M$ a valores em $W$ fazendo:

$$
(T \circ \omega)_{m}\left(v_{1}, \ldots, v_{r}\right)=T\left(\omega_{m}\left(v_{1}, \ldots, v_{r}\right)\right)
$$

para $m \in M, v_{1}, \ldots, v_{r} \in T_{m} M$. Essa construção não aparece no caso de formas diferenciais no sentido usual (i.e., a valores reais).

As operações acima satisfazem as propriedades usuais. As demonstrações são bastante simples (em geral pode-se usar uma base em $V$ para recair no caso de formas a valores reais). Temos que a diferenciação exterior $\omega \mapsto d \omega$, o pull-back $\omega \mapsto f^{*} \omega$ e a composição $\omega \mapsto T \circ \omega$ são todas operações $\mathbb{R}$-lineares e comutam entre si. Com relação ao produto exterior temos que $f^{*}(\omega \wedge \mu)=\left(f^{*} \omega\right) \wedge\left(f^{*} \mu\right)$ e $d(\omega \wedge \mu)=d \omega \wedge \mu+(-1)^{r} \omega \wedge d \mu$, onde $\omega$ é uma $r$-forma. A última fórmula pode ser demonstrada observando que $(\omega \wedge \mu)^{k}=\sum_{i, j} p_{i j}^{k} \omega^{i} \wedge \mu^{j}$, escolhendo bases nos espaços vetoriais envolvidos (onde $p_{i j}^{k}$ é uma representação matricial do produto bilinear $p$ nessas bases).

Enunciamos também a fórmula de Cartan para diferenciação exterior em termos de colchetes de Lie (que pode ser provada coordenada por coordenada também). Se $\theta$ é uma 1-forma a valores em $V$ então temos:

$$
d \theta(X, Y)=X(\theta(Y))-Y(\theta(X))-\theta([X, Y])
$$

para quaisquer campos vetoriais $X, Y$ em $M$ (se $f: M \rightarrow V$ é uma função então podemos definir uma função $X(f): M \rightarrow V$ fazendo $X(f)(m)=d f_{m}\left(X_{m}\right)$ para $\left.m \in M\right)$.

Finalmente, se $\theta$ é uma 1-forma a valores numa álgebra de Lie $\mathfrak{g}$ então podemos definir uma 2-forma $\theta \wedge \theta$ fazendo:

$$
(\theta \wedge \theta)_{m}(v, w)=\left[\theta_{m}(v), \theta_{m}(w)\right]
$$

para $m \in M, v, w \in T_{m} M$.

Observação 2.3. Considerando a 1-forma $\theta$ tomando valores na álgebra associativa envolvente de $\mathfrak{g}$ então a definição acima segue da definição geral da multiplicação exterior, mas preferimos evitar essa linguagem.

Seja $G$ um grupo de Lie. A forma de Maurer-Cartan de $G$ é a 1-forma $\Theta$ a valores em $\mathfrak{g}$ definida por $\Theta_{g}(v)=g^{-1} v$ para $g \in G, v \in T_{g} G$ ( $\Theta$ é a única 1-forma invariante à esquerda tal que $\Theta_{1}: \mathfrak{g} \rightarrow \mathfrak{g}$ é a identidade). Se $X, Y$ são campos invariantes à esquerda em $G$ então:

$$
d \Theta(X, Y)=X(\Theta(Y))-Y(\Theta(X))-\Theta([X, Y])=-\Theta([X, Y])
$$


pois $\Theta(X)$ e $\Theta(Y)$ são funções constantes. Daí para $g \in G$ e $v, w \in T_{g} G$, escolhemos campos invariantes à esquerda com $X_{g}=v$ e $Y_{g}=w$, aplicamos a identidade (2.1) e obtemos:

$$
d \Theta_{g}(v, w)=-\left[\Theta_{g}(v), \Theta_{g}(w)\right]
$$

Segue então a equação fundamental de Cartan:

$$
d \Theta=-\Theta \wedge \Theta
$$

Seja agora $G$ um grupo de Lie, $K$ um subgrupo fechado de $G$ e $\mathfrak{d} \subset \mathfrak{g} / \mathfrak{k}$ um subespaço $\overline{\mathrm{Ad}}$-invariante. Denotamos por $\mathcal{D}$ a distribuição $G$-invariante em $G / K \operatorname{com} \mathcal{D}_{1 K}=\mathfrak{d}$. Seja $s: U \rightarrow G$ uma seção local definida num aberto $U \subset G / K$. Defina:

$$
\begin{aligned}
f: U \times(\mathfrak{g} / \mathfrak{k})^{*} & \rightarrow T(G / K)_{\mid U}^{*} \\
(g K, \alpha) & \mapsto d \bar{l}_{s(g K)}(1 K)^{*-1}(\alpha) .
\end{aligned}
$$

Temos que $f$ é um difeomorfismo (na verdade, $f$ é uma trivialização do fibrado vetorial $T(G / K)^{*}$ sobre o aberto $\left.U\right)$. Observe que $f$ faz a subvariedade $U \times \mathfrak{d}^{0}$ de $U \times(\mathfrak{g} / \mathfrak{k})^{*}$ corresponder à subvariedade $\mathcal{D}_{\mid U}^{0}$ de $T(G / K)_{\mid U}^{*}$, onde $\mathfrak{d}^{0} \subset(\mathfrak{g} / \mathfrak{k})^{*}$ é o anulador do subespaço $\mathfrak{d} \subset \mathfrak{g} / \mathfrak{k}$ (isso segue diretamente da $G$-invariância da distribuição $\mathcal{D}$ ).

Determinamos agora $f^{*} \theta$ e $f^{*} \omega$, onde $\theta$ e $\omega$ são as formas canônicas de $T(G / K)^{*}$ introduzidas na seção 1. Para $g K \in U, \alpha \in(\mathfrak{g} / \mathfrak{k})^{*}, v \in T_{g K}(G / K)$ e $\beta \in(\mathfrak{g} / \mathfrak{k})^{*}$ temos:

$$
\left(f^{*} \theta\right)_{(g K, \alpha)}(v, \beta)=\theta_{f(g K, \alpha)} \circ d f_{(g K, \alpha)}(v, \beta)=d \bar{l}_{s(g K)}(1 K)^{*^{-1}}(\alpha) \circ d \pi_{f(g K, \alpha)} \circ d f_{(g K, \alpha)}(v, \beta),
$$

onde utilizamos simplesmente a definição de $\theta$ dada em (1.1). Aplicando a regra da cadeia e observando que $\pi \circ f$ é simplesmente a primeira projeção do produto $U \times(\mathfrak{g} / \mathfrak{k})^{*}$ vem:

$$
\left(f^{*} \theta\right)_{(g K, \alpha)}(v, \beta)=\alpha \circ d \bar{l}_{s(g K)}(1 K)^{-1}(v)=\alpha\left(s(g K)^{-1} v\right) .
$$

Considere a 1-forma $\bar{\pi} \circ s^{*} \Theta$ em $U \subset G / K$ a valores em $\mathfrak{g} / \mathfrak{k}$. Temos, para $g K \in U$, $v \in T_{g K}(G / K)$ :

$$
\left(\bar{\pi} \circ s^{*} \Theta\right)_{g K}(v)=\bar{\pi} \circ \Theta_{s(g K)} \circ d s_{g K}(v)=d \pi_{1} \circ d l_{s(g K)}(1)^{-1} \circ d s_{g K}(v) .
$$

Utilizando a regra da cadeia e observando que $\pi \circ l_{s(g K)}^{-1} \circ s=\bar{l}_{s(g K)}^{-1}$ obtemos:

$$
\left(\bar{\pi} \circ s^{*} \Theta\right)_{g K}(v)=\bar{\pi}\left(s(g K)^{-1} d s_{g K}(v)\right)=s(g K)^{-1} v .
$$

Denote por $\pi_{1}$ e $\pi_{2}$ as projeções do produto cartesiano $U \times(\mathfrak{g} / \mathfrak{k})^{*}$. Observe que $\pi_{2}$ é uma 0 -forma em $U \times(\mathfrak{g} / \mathfrak{k})^{*}$ a valores em $(\mathfrak{g} / \mathfrak{k})^{*}$. Temos um operador bilinear:

$$
\begin{gathered}
p:(\mathfrak{g} / \mathfrak{k})^{*} \times(\mathfrak{g} / \mathfrak{k}) \rightarrow \mathbb{R} \\
(\alpha, v) \mapsto \alpha(v),
\end{gathered}
$$


em relação ao qual fazemos o produto exterior de uma forma a valores em $\mathfrak{g} / \mathfrak{k}$ por uma forma a valores em $(\mathfrak{g} / \mathfrak{k})^{*}$ obtendo uma forma a valores reais.

Substituindo (2.5) em (2.4) obtemos:

$$
f^{*} \theta=\pi_{2} \wedge \pi_{1}^{*}\left(\bar{\pi} \circ s^{*} \Theta\right) .
$$

Aplicando a diferenciação exterior em ambos os lados vem:

$$
-f^{*} \omega=d \pi_{2} \wedge \pi_{1}^{*}\left(\bar{\pi} \circ s^{*} \Theta\right)+\pi_{2} \wedge \pi_{1}^{*}\left(\bar{\pi} \circ s^{*} d \Theta\right) .
$$

Podemos então utilizar a equação fundamental de Cartan (2.2):

$$
f^{*} \omega=-d \pi_{2} \wedge \pi_{1}^{*}\left(\bar{\pi} \circ s^{*} \Theta\right)+\pi_{2} \wedge \pi_{1}^{*}\left(\bar{\pi} \circ s^{*}(\Theta \wedge \Theta)\right) .
$$

Mais explicitamente, se $g K \in U, \alpha, \beta_{1}, \beta_{2} \in(\mathfrak{g} / \mathfrak{k})^{*}$ e $v_{1}, v_{2} \in T_{g K}(G / K)$ então:

$$
\begin{aligned}
\left(f^{*} \omega\right)_{(g K, \alpha)}\left(\left(v_{1}, \beta_{1}\right),\left(v_{2}, \beta_{2}\right)\right)= & -\beta_{1} \circ \bar{\pi} \circ \Theta_{s(g K)} \circ d s_{g K}\left(v_{2}\right)+\beta_{2} \circ \bar{\pi} \circ \Theta_{s(g K)} \circ d s_{g K}\left(v_{1}\right) \\
& +\alpha \circ \bar{\pi}\left[\Theta_{s(g K)} \circ d s_{g K}\left(v_{1}\right), \Theta_{s(g K)} \circ d s_{g K}\left(v_{2}\right)\right] .
\end{aligned}
$$

Por simplicidade escrevemos:

$$
\hat{v}_{i}=s(g K)^{-1} d s_{g K}\left(v_{i}\right) \in \mathfrak{g} \quad \text { e } \quad w_{i}=\bar{\pi}\left(\hat{v}_{i}\right)=s(g K)^{-1} v_{i} \in \mathfrak{g} / \mathfrak{k}, \quad i=1,2,
$$

onde na segunda igualdade utilizamos (2.5). Temos então:

$$
\left(f^{*} \omega\right)_{(g K, \alpha)}\left(\left(v_{1}, \beta_{1}\right),\left(v_{2}, \beta_{2}\right)\right)=-\beta_{1}\left(w_{2}\right)+\beta_{2}\left(w_{1}\right)+\alpha \circ \bar{\pi}\left[\hat{v}_{1}, \hat{v}_{2}\right] .
$$

Recordando que $f$ leva $U \times \mathfrak{d}^{0}$ sobre $\mathcal{D}_{\mid U}^{0}$, segue que o núcleo de $\omega_{\mid \mathcal{D}^{0}}$ corresponde pela diferencial de $f$ ao núcleo de $\left(f^{*} \omega\right)_{\mid U \times 0^{0}}$, o qual calculamos a seguir.

Supomos agora $\alpha \in \mathfrak{d}^{0}$ e queremos determinar os pares $\left(v_{1}, \beta_{1}\right) \in T_{g K}(G / K) \times \mathfrak{d}^{0}$ que anulam (2.7) sempre que $v_{2} \in T_{g K}(G / K)$ e $\beta_{2} \in \mathfrak{o}^{0}$. Fazendo $v_{2}=0$ e deixando $\beta_{2} \in \mathfrak{o}^{0}$ arbitrário vemos que necessariamente $w_{1} \in \mathfrak{d}$. Segue que $\left(v_{1}, \beta_{1}\right) \in T_{g K}(G / K) \times \mathfrak{d}^{0}$ pertence ao núcleo de $\left(f^{*} \omega\right)_{\mid U \times 0^{\circ}}$ no ponto $(g K, \alpha)$ se e somente se:

$$
w_{1} \in \mathfrak{d} \quad \text { e } \quad \beta_{1}\left(w_{2}\right)=\alpha \circ \bar{\pi}\left[\hat{v}_{1}, \hat{v}_{2}\right]
$$

para todo $v_{2} \in T_{g K}(G / K)$, onde $\hat{v}_{i}, w_{i}$ são definidos como em (2.6).

Escolhemos agora um subespaço complementar arbitrário $\mathfrak{m}$ de $\mathfrak{k}$ em $\mathfrak{g}$. Daí $\mathfrak{g}=\mathfrak{k} \oplus \mathfrak{m}$ e identificamos $\mathfrak{g} / \mathfrak{k}$ com $\mathfrak{m}$ através de $\bar{\pi}_{\mid \mathfrak{m}}$. A projeção $\bar{\pi}$ fica identificada então com a projeção $\pi_{\mathfrak{m}}: \mathfrak{g} \rightarrow \mathfrak{m}$ em relação a decomposição $\mathfrak{g}=\mathfrak{k} \oplus \mathfrak{m}$. Consideramos agora $\mathfrak{d} \subset \mathfrak{m}$ e $\mathfrak{d}^{0} \subset \mathfrak{m}^{*}$. Observe que o subespaço $\bar{\pi}^{-1}(\mathfrak{d}) \subset \mathfrak{g}$ fica agora identificado com $\mathfrak{k}+\mathfrak{d}$. A observação 2.2 nos diz agora que $[\mathfrak{k}, \mathfrak{k}+\mathfrak{d}] \subset \mathfrak{k}+\mathfrak{o}$.

Observe que os vetores $\hat{v}_{i}$ e $w_{i}$ definidos em (2.6) diferem por um elemento de $\mathfrak{k}$, pois agora $w_{i}=\pi_{\mathrm{m}}\left(\hat{v}_{i}\right)$. Supondo $w_{1} \in \mathfrak{d}$, temos que $\hat{v}_{1} \in \mathfrak{k}+\mathfrak{d}$ e portanto $\left[\hat{v}_{1}, \hat{v}_{2}\right]$ e $\left[\hat{v}_{1}, w_{2}\right]$ diferem por um elemento de $\mathfrak{k}+\mathfrak{d}$. Como $\alpha \in \mathfrak{m}^{*}$ anula $\mathfrak{d}$, podemos substituir $\hat{v}_{2}$ por $w_{2}$ em (2.8). É claro que quando $v_{2}$ percorre todo o espaço tangente $T_{g K}(G / K)$ então $w_{2}$ percorre o espaço $\mathfrak{g} / \mathfrak{k} \cong \mathfrak{m}$. Finalmente, obtemos que (2.8) equivale a:

$$
w_{1} \in \mathfrak{d} \quad \text { e } \quad \beta_{1}=\left(\alpha \circ \pi_{\mathrm{m}} \circ \operatorname{ad}_{\hat{v}_{1}}\right)_{\mid \mathrm{m}} .
$$

Resumimos esses cálculos no seguinte teorema. 
Teorema 2.1. Sejam $G$ um grupo de Lie, $K \subset G$ um subgrupo fechado e $\mathcal{D}$ uma distribuição $G$-invariante em $G / K$. Escolha um subespaço complementar arbitrário $\mathrm{m}$ de k em $\mathfrak{g}$ e uma seção local $s: U \rightarrow G$ definida num aberto $U \subset G / K$. Seja $\mathfrak{d}=\mathcal{D}_{1 K} \subset \mathfrak{g} / \mathfrak{k} \cong \mathfrak{m}$ e seja $f$ o difeomorfismo definido em (2.3). Denote por $\omega$ a forma simplética canônica de $T(G / K)^{*}$. Fixados $g K \in U, \alpha \in \mathfrak{d}^{0}$, temos que um vetor $(v, \beta) \in T_{g K}(G / K) \times \mathfrak{d}^{0}$ pertence ao núcleo de $\left(f^{*} \omega\right)_{\mid U \times 0^{\circ}}$ no ponto $(g K, \alpha)$ se e somente se:

$$
w \in \mathfrak{d} \quad \text { e } \quad \beta=\left(\pi_{\mathfrak{m}} \circ \operatorname{ad}_{\hat{v}} \circ i_{\mathfrak{m}}\right)^{*} \alpha
$$

onde $\hat{v}=s(g K)^{-1} d s_{g K}(v), w=\pi_{\mathfrak{m}}(\hat{v}), \pi_{\mathfrak{m}}: \mathfrak{g} \rightarrow \mathfrak{m}$ é a projeção em relação à decomposição $\mathfrak{g}=\mathfrak{k} \oplus \mathfrak{m}$ e $i_{\mathfrak{m}}: \mathfrak{m} \rightarrow \mathfrak{g}$ é a inclusão.

Demonstração. Segue diretamente de (2.9).

Observação 2.4. No teorema anterior, identificando $\mathfrak{g} / \mathfrak{k} \cong \mathfrak{m}$, segue de (2.5) que $w$ é simplesmente $s(g K)^{-1} v$, donde a condição $w \in \mathfrak{d}$ nos diz simplesmente que $v$ pertence à distribuição $\mathcal{D}$.

Corolário. Nas condições do teorema, seja $\gamma:[a, b] \rightarrow M$ uma curva absolutamente contínua com $\gamma([a, b]) \subset U$. Então $\gamma$ é um extremal anormal de $(G / K, \mathcal{D})$ (com qualquer métrica sub-Riemanniana) se e somente se existe uma função absolutamente contínua que nunca se anula $\lambda:[a, b] \rightarrow \mathfrak{d}^{0}$ tal que:

$$
\mu(t) \in \mathfrak{d} \quad \text { e } \quad \dot{\lambda}(t)=\left(\pi_{\mathfrak{m}} \circ \operatorname{ad}_{\hat{\mu}(t)} \circ i_{\mathfrak{m}}\right)^{*} \lambda(t),
$$

para quase todo $t \in[a, b]$, onde escrevemos:

$$
\hat{\mu}(t)=s(\gamma(t))^{-1} d s_{\gamma(t)}(\dot{\gamma}(t)) \quad \text { e } \quad \mu(t)=\pi_{\mathfrak{m}}(\hat{\mu}(t)) .
$$

Demonstração. As curvas absolutamente contínuas $\eta:[a, b] \rightarrow \mathcal{D}^{0}$ que se projetam sobre $\gamma$ estão em correspondência biunívoca com as curvas absolutamente contínuas $\lambda:[a, b] \rightarrow d^{0}$ fazendo $\eta=f \circ(\gamma, \lambda)$. Temos que $\gamma$ é um extremal anormal se e somente se existe uma característica $\eta:[a, b] \rightarrow \mathcal{D}^{0}$ de $\mathcal{D}$ (i.e., $\dot{\eta}(t)$ pertence ao núcleo de $\omega_{\mid \mathcal{D}^{0}}$ no ponto $\eta(t)$ para quase todo $t \in[a, b])$ que nunca se anula e que se projeta sobre $\gamma$. O resultado segue diretamente do teorema.

Observação 2.5. Analogamente à observação 2.4, temos que no corolário anterior $\mu(t)$ é simplesmente $s(\gamma(t))^{-1} \dot{\gamma}(t)$, quando identificamos $\mathfrak{g} / \mathfrak{k} \cong \mathfrak{m}$. Daí a condição $\mu(t) \in \mathfrak{o}$ nos diz simplesmente que $\gamma$ é horizontal. Observamos também que podemos substituir no corolário a condição " $\lambda$ nunca se anula" por " $\lambda$ não é identicamente nula" (vide observação 1.4). 


\section{Preliminares algébricos.}

Nesta seção expomos alguns preliminares algébricos que serão usados na seção 4.

Na subseção 3.1 expomos alguns resultados simples de álgebra linear que serão necessários posteriormente. Resumimos também a teoria de formas reais e complexificações de espaços vetorias e álgebras de Lie. Na subseção 3.2 expomos resumidamente a teoria de estrutura de raízes e decomposição de Cartan de álgebras de Lie complexas semi-simples. A exposição não é totalmente auto-contida e referimos o leitor para [Vj], para mais detalhes. Na subseção 3.3 introduzimos o conceito de álgebras de Lie involutivas ortogonais, que serão essenciais para a descrição da classe de variedades sub-Riemannianas que aparece no teorema fundamental na seção 4. A exposição feita na subseção 3.3 é auto-contida, mas referimos o leitor para [W], para mais detalhes. 


\subsection{Recapitulação de álgebra linear.}

Seja $V$ um espaço vetorial sobre um corpo $\mathbb{K}$ (suporemos sempre $\mathbb{K}=\mathbb{R}$ ou $\mathbb{K}=\mathbb{C}$, mesmo quando isso não for estritamente necessário). Todos os espaços vetoriais (e álgebras de Lie) envolvidos nesta seção e nas próximas serão de dimensão finita, exceto quando for feita menção explícita em contrário.

Seja $B: V \times V \rightarrow \mathbb{K}$ uma forma bilinear simétrica. Se $W \subset V$ é um subespaço definimos o complemento $B$-ortogonal de $W$ em $V$ (ou simplesmente complemento ortogonal, quando $B$ estiver subentendida) fazendo:

$$
W^{\perp}=\{v \in V: B(v, w)=0, \forall w \in W\} .
$$

O núcleo de $B$ é por definição o subespaço $V^{\perp}$. Dizemos que $B$ é não degenerada quando seu núcleo for nulo. Isso equivale a dizer que a aplicação $v \in V \mapsto B(v, \cdot) \in V^{*}$ é um isomorfismo. Note que em geral não é verdade que $W \cap W^{\perp}=0$ nem que $V=W+W^{\perp}$. Temos porém o seguinte lema.

Lema 3.1.1. Seja $V$ um espaço vetorial e $B$ uma forma bilinear simétrica em $V$. Um subespaço $W \subset V$ satisfaz $V=W \oplus W^{\perp}$ se e somente se a restrição de $B$ a $W$ é não degenerada.

Demonstração. É fácil ver que o núcleo da restrição de $B$ a $W$ é $W \cap W^{\perp}$, de modo que se $V=W \oplus W^{\perp}$ então a restrição de $B$ a $W$ é não degenerada. Reciprocamente, se a restrição de $B$ a $W$ é não degenerada então $W \cap W^{\perp}=0$ e resta mostrar que $V=W+W^{\perp}$. Seja $v \in V$. Defina $\alpha \in W^{*}$ fazendo $\alpha(w)=B(v, w)$. Pelo comentário que precede o lema existe um único $w \in W$ tal que $B(w, \cdot)_{\mid W}=\alpha$. Segue que $v-w \in W^{\perp}$, o que completa a demonstração.

Se $V$ é um espaço vetorial complexo, uma forma real em $V$ é um subespaço real $V^{0} \subset V$ tal que $V=V^{0} \oplus i V^{0}$. Por exemplo, $\mathbb{R}^{n}$ é uma forma real em $\mathbb{C}^{n}$. Fixada uma forma real em $V$ temos um único isomorfismo $\mathbb{R}$-linear $T: V \rightarrow V$ tal que $T_{\mid V^{\circ}}=$ Id e $T_{\mid i V^{0}}=-$ Id. Escrevemos $T(v)=\bar{v}$ e dizemos que $\bar{v}$ é o conjugado de $v \in V$ em relação à forma real $V^{0}$.

Sejam $V_{1}, \ldots, V_{n}, W$ espaços vetoriais complexos com formas reais $V_{1}^{0}, \ldots, V_{n}^{0}, W^{0}$ respectivamente. Dizemos que um operador $n$-linear (sobre $\mathbb{C}$ ) $B: V_{1} \times \cdots \times V_{n} \rightarrow W$ preserva as formas reais quando $B\left(V_{1}^{0} \times \cdots \times V_{n}^{0}\right) \subset W^{0}$. Toda base (sobre $\mathbb{R}$ ) de uma forma real $V^{0} \subset V$ é também uma base (sobre $\mathbb{C}$ ) de $V$ (na verdade, uma forma real pode ser construida considerando-se o subespaço real gerado por uma base de $V$ sobre $\mathbb{C}$ ). Numa tal base, um operador multi-linear que preserva as formas reais é caracterizado pelo fato que seus coeficientes matriciais são reais. Bases de $V$ obtidas assim são chamadas adaptadas à forma real $V^{0}$. Mais geralmente podemos definir um operador $n$-linear (sobre C) $\bar{B}$ fazendo:

$$
\bar{B}\left(v_{1}, \ldots, v_{n}\right)=\overline{B\left(\bar{v}_{1}, \ldots, \bar{v}_{n}\right)} .
$$

Os coeficientes de $\bar{B}$ numa base adaptada à forma real são conjugados aos coeficientes de $B$. Temos que $B$ preserva as formas reais se e somente se $B=\bar{B}$.

Se $V$ é um espaço vetorial real então uma complexificação de $V$ é um espaço vetorial complexo $V^{c}$ que contém $V$ como forma real. Podemos construir $V^{c}$ fazendo $V^{c}=V \otimes \mathbb{C}$, 
definindo $i(v \otimes z)=v \otimes(i z)$ e identificando $V \operatorname{com} V \otimes 1$. Podemos também simplesmente fazer $V^{c}=V \oplus V$, definir $i(v, w)=(-w, v)$ e identificar $V \operatorname{com} V \oplus 0$. A complexificação $V^{c}$ é única no sentido que duas delas são isomorfas por um isomorfismo $\mathbb{C}$-linear que fixa $V$.

Se $B: V_{1} \times \cdots \times V_{n} \rightarrow W$ é um operador $n$-linear onde cada $V_{i}$ é um espaço vetorial real e $W$ é um espaço vetorial complexo, existe uma única extensão de $B$ a $V_{1}^{c} \times \cdots \times V_{n}^{c}$ que é $n$-linear sobre $\mathbb{C}$. Quando $W$ também é apenas real, podemos estender $B$ se considerarmos seu contra-domínio em $W^{c}$. Essa extensão será denotada por $B^{c}$ (às vezes escreveremos apenas $B$ em vez de $B^{c}$, por simplicidade). Se $B: V \times V \rightarrow \mathbb{R}$ é uma forma bilinear simétrica então $B^{c}: V^{c} \times V^{c} \rightarrow \mathbb{C}$ é também uma forma bilinear simétrica. Temos que $B$ é não degenerada se e somente se $B^{c}$ é não degenerada.

Se $W \subset V$ é um subespaço real então o subespaço complexo gerado por $W$ em $V^{c}$ é uma complexificação de $W$ e é denotado portanto por $W^{c}$. Em geral, um subespaço complexo $Z \subset V^{c}$ é da forma $W^{c}$ para algum $W \subset V$ se e somente se $Z$ é invariante por conjugação, i.e., $\bar{Z}=Z$ (ou, mais explicitamente, $v \in Z \Rightarrow \bar{v} \in Z$ ). Nesse caso temos $W=Z \cap V$.

Observe que um produto interno num espaço real $V$ admite uma única extensão $\mathbb{C}$-bilinear a $V^{c}$, a qual é uma forma bilinear simétrica não degenerada. É possível no entanto obter uma única extensão sesquilinear (i.e., linear conjugada na segunda variável e $\mathbb{C}$-linear na primeira variável). A mesma será um produto hermiteano em $V^{c}$.

Se g é uma álgebra de Lie real então o colchete de Lie admite uma única extensão $\mathbb{C}$-bilinear a $\mathfrak{g}^{c}$. Obtemos então em $\mathfrak{g}^{c}$ uma estrutura de álgebra de Lie complexa, à qual chamamos a complexificação da álgebra de Lie g. Note que o colchete de Lie preserva a forma real e portanto vale a identidade $\overline{[X, Y]}=[\bar{X}, \bar{Y}]$. Observe que reciprocamente, se $\mathfrak{g}$ é uma álgebra de Lie complexa e $\mathfrak{g}^{0} \subset \mathfrak{g}$ é uma forma real preservada pelo colchete de Lie então $\mathfrak{g}^{0}$ é uma álgebra de Lie real e $\mathfrak{g}$ é uma complexificação de $\mathfrak{g}^{0}$.

Precisamos agora de alguns lemas sobre diagonalização de operadores lineares. Seja $V$ um espaço vetorial complexo. Sabe-se que um operador linear $T: V \rightarrow V$ é diagonalizável se e somente se seu polinômio minimal $m(T) \in \mathbb{C}[X]$ (i.e., o gerador do ideal principal de polinômios em $\mathbb{C}[X]$ que anulam $T$ ) possui apenas raízes de multiplicidade 1 . Temos o seguinte lema:

Lema 3.1.2. Seja $T: V \rightarrow V$ um operador linear diagonalizável num espaço vetorial complexo $V$. Se $W \subset V$ é um subespaço invariante, i.e., $T(W) \subset W$ então $T_{\mid W}: W \rightarrow W$ também é diagonalizável.

Demonstração. Se $p(X) \in \mathbb{C}[X]$ é um polinômio então $p\left(T_{\mid W}\right)=p(T)_{\mid W}$. Segue então que $m(T)$ anula $T_{\mid W}$ e portanto $m\left(T_{\mid W}\right)$ divide $m(T)$. Segue que todas as raízes de $m\left(T_{\mid W}\right)$ tem multiplicidade 1.

Dizemos que um conjunto $\mathcal{T}$ de operadores lineares de $V$ é simultaneamente diagonalizável quando existir uma base de $V$ que diagonaliza todos os elementos de $\mathcal{T}$. Segue então que os elementos de $\mathcal{T}$ são individualmente diagonalizáveis e comutam entre si. O resultado seguinte fornece a recíproca. Observamos que se $T$ e $S$ comutam então os auto-espaços de $T$ são $S$-invariantes. 
Lema 3.1.3. Seja $V$ um espaço vetorial complexo e seja $\mathcal{T}$ um conjunto de operadores lineares diagonalizáveis em $V$ que comutam dois a dois. Então $\mathcal{T}$ é simultaneamente diagonalizável.

Demonstração. Usamos indução na dimensão de $V$. O resultado é óbvio para dimensão 0 (e 1). Supomos o resultado válido para espaços vetoriais de dimensão menor que a de $V$. Se todo elemento de $\mathcal{T}$ é múltiplo da identidade, o resultado é óbvio. Suponha então que existe $T \in \mathcal{T}$ que não é múltiplo da identidade. Os auto-espaços de $T$ são então subespaços próprios de $V$ invariantes por todos os elementos de $\mathcal{T}$. Podemos escrever então $V=V_{1} \oplus V_{2}$, com $\operatorname{dim} V_{1}<\operatorname{dim} V, \operatorname{dim} V_{2}<\operatorname{dim} V$ e $V_{1}, V_{2}$ invariantes por todos os elementos de $\mathcal{T}$. Pelo lema 3.1.2 as restrições dos elementos de $\mathcal{T}$ a $V_{1}$ e $V_{2}$ são ainda diagonalizáveis (e comutam) e são então simultaneamente diagonalizáveis pela hipótese de indução.

Corolário. Seja $\mathfrak{g}$ uma álgebra de Lie complexa $\mathrm{e} \mathfrak{h} \subset \mathfrak{g}$ uma subálgebra abeliana. Se $S$ é um conjunto de geradores de $\mathfrak{h}$ (como espaço vetorial) e se $\operatorname{ad}_{X}: \mathfrak{g} \rightarrow \mathfrak{g}$ é diagonalizável para todo $X \in S$ então $\operatorname{ad}_{X}$ é diagonalizável para todo $X \in \mathfrak{h}$.

Demonstração. Para $X, Y \in S$ temos $\left[\operatorname{ad}_{X}, \operatorname{ad}_{Y}\right]=\operatorname{ad}_{[X, Y]}=0$, donde o conjunto:

$$
\mathcal{T}=\left\{\operatorname{ad}_{X}: X \in S\right\},
$$

satisfaz as hipóteses do lema. Para $X \in \mathfrak{h}$ temos que ad $X$ é uma combinação linear dos elementos de $\mathcal{T}$, donde uma base que diagonaliza simultaneamente os elementos de $\mathcal{T}$ também diagonaliza $\operatorname{ad}_{X}$. 


\subsection{Estrutura de álgebras de Lie complexas semi-simples.}

Seja $\mathfrak{g}$ uma álgebra de Lie sobre $\mathbb{K}$. A forma de Killing de $\mathfrak{g}$ é a forma bilinear simétrica $\mathcal{K}: \mathfrak{g} \times \mathfrak{g} \rightarrow \mathbb{K}$ definida por $\mathcal{K}(X, Y)=\operatorname{tr}\left(\operatorname{ad}_{X} \operatorname{ad}_{Y}\right)$, onde $\operatorname{tr} T$ denota o traço de um operador linear $T: \mathfrak{g} \rightarrow \mathfrak{g}$. Sejam $\sigma: \mathfrak{g} \rightarrow \mathfrak{g}$ um automorfismo e $d: \mathfrak{g} \rightarrow \mathfrak{g}$ uma derivação de $\mathfrak{g}$ (i.e., $\sigma[X, Y]=[\sigma(X), \sigma(Y)]$ e $d[X, Y]=[d(X), Y]+[X, d(Y)]$, para $X, Y \in \mathfrak{g}$ ). Então:

$$
\mathcal{K}(\sigma(X), \sigma(Y))=\mathcal{K}(X, Y) \quad \text { e } \quad \mathcal{K}(d(X), Y)+\mathcal{K}(X, d(Y))=0,
$$

para $X, Y \in \mathfrak{g}$. Dizemos também que $\sigma$ é $\mathcal{K}$-ortogonal e $d$ é $\mathcal{K}$-antisimétrica. A segunda fórmula é especialmente interessante quando $d=\operatorname{ad}_{Z}$ para algum $Z \in \mathfrak{g}$, i.e., $d$ é uma derivação interna.

Dado um subconjunto $S \subset \mathfrak{g}$, definimos o centralizador de $S$ fazendo:

$$
\mathfrak{z}(S)=\{X \in \mathfrak{g}:[X, Y]=0, \forall Y \in S\} .
$$

Segue facilmente da identidade de Jacobi que $\mathfrak{z}(S)$ é uma subálgebra de g. Quando $X$ está em $\mathfrak{z}(S)$ dizemos também que $X$ centraliza $S$. Em geral, se $\mathfrak{h} \subset \mathfrak{g}$ é uma subálgebra de $\mathfrak{g}$ definimos o centralizador de $S$ em $\mathfrak{h}$ fazendo $\mathfrak{z}_{\mathfrak{h}}(S)=\mathfrak{z}(S) \cap \mathfrak{h}$. O centro de $\mathfrak{g}$ é o ideal $\mathfrak{z}=\mathfrak{z}(\mathfrak{g})$. Para $X \in \mathfrak{g}$ escrevemos $\mathfrak{z}(X)$ em vez de $\mathfrak{z}(\{X\})$. É claro que $X \in \mathfrak{z}(S)$ se e somente se $S \subset \mathfrak{z}(X)$. Como $\mathfrak{z}(X)$ é uma subálgebra de $\mathfrak{g}$, segue que o centralizador de $S$ coincide com o centralizador da subálgebra de Lie gerada por $S$ em g. Suponha agora que $\mathfrak{g}$ é real. Se $\mathfrak{g}^{c}$ é a complexificação de $\mathfrak{g}$ então para $S \subset \mathfrak{g}$ vale $\mathfrak{z}_{\mathfrak{g}^{c}}(S)=\mathfrak{z}_{\mathfrak{g}}(S)^{c}$. Em particular, $\mathfrak{z}_{\mathfrak{g}^{c}}\left(\mathfrak{g}^{c}\right)=\mathfrak{z}_{\mathfrak{g}^{c}}(\mathfrak{g})=\mathfrak{z}_{\mathfrak{g}}(\mathfrak{g})^{c}$, i.e., o centro de $\mathfrak{g}^{c}$ é a complexificação do centro de g.

Diremos que uma álgebra de Lie $\mathfrak{g}$ (real ou complexa) é semi-simples quando sua forma de Killing $\mathcal{K}$ for não degenerada. Sabe-se que g é semi-simples se e somente se não possui ideais abelianos não nulos (o que também equivale a dizer que $\mathfrak{g}$ não possui ideais solúveis não nulos, mas esse fato não será utilizado). Em particular uma álgebra de Lie semi-simples não possui centro.

Se $\mathfrak{g}$ é uma álgebra de Lie real e se $\mathcal{K}$ é sua forma de Killing então a forma de Killing de $\mathfrak{g}^{c}$ é simplesmente $\mathcal{K}^{c}$. Segue que $\mathfrak{g}$ é semi-simples se e somente se $\mathfrak{g}^{c}$ é semi-simples.

Seja $\mathfrak{g}$ uma álgebra de Lie complexa semi-simples. Uma subálgebra de Cartan de $\mathfrak{g}$ é uma subálgebra abeliana maximal $\mathfrak{h} \subset \mathfrak{g}$ (i.e., $\mathfrak{h}$ é uma subálgebra abeliana de $\mathfrak{g}$ que não está propriamente contida em nenhuma subálgebra abeliana de $\mathfrak{g}$ ) tal que $\operatorname{ad}_{X}: \mathfrak{g} \rightarrow \mathfrak{g}$ é diagonalizável para todo $X \in \mathfrak{h}$. Temos que subálgebras de Cartan realmente existem (e duas delas são conjugadas por automorfismos internos de $\mathfrak{g}$, mas esse fato não será usado. Segue daí que todas as subálgebras de Cartan possuem a mesma dimensão e essa é chamada o posto de $\mathfrak{g})$.

Observe que $\mathfrak{h}$ é abeliana maximal se e somente se $\mathfrak{z}(\mathfrak{h})=\mathfrak{h}$ (observe que se existisse $X \in \mathfrak{z}(\mathfrak{h}), X \notin \mathfrak{h}$ então $\mathfrak{h}$ e $X$ gerariam uma subálgebra abeliana maior que $\mathfrak{h})$. Note também que pelo corolário do lema 3.1 .3 , para ver que $\operatorname{ad}_{X}$ é diagonalizável para todo $X \in \mathfrak{h}$ basta considerar os elementos $X$ de um conjunto de geradores de $\mathfrak{h}$ (como espaço vetorial).

Fixamos a partir de agora uma subálgebra de Cartan $\mathfrak{h} \subset \mathfrak{g}$. Seja $\alpha \in \mathfrak{h}^{*}$. Defina um subespaço $\mathfrak{g}_{\alpha}$ de $\mathfrak{g}$ fazendo:

$$
\mathfrak{g}_{\alpha}=\{X \in \mathfrak{g}:[H, X]=\alpha(H) X, \forall H \in \mathfrak{h}\} .
$$


Como $\mathfrak{h}$ é abeliana maximal segue que $\mathfrak{g}_{0}=\mathfrak{h}$. Se $\alpha \in \mathfrak{h}^{*}$ é tal que $\mathfrak{g}_{\alpha} \neq 0$ e $\alpha \neq 0$, dizemos que $\alpha$ é uma raiz de g. Denotamos por $\Delta$ o conjunto das raízes de g. Temos que $\Delta$ é finito e vale a seguinte decomposição:

$$
\mathfrak{g}=\mathfrak{h} \oplus \bigoplus_{\alpha \in \Delta} \mathfrak{g}_{\alpha}
$$

chamada a decomposição de Cartan de $\mathfrak{g}$ (isso é uma conseqüência relativamente simples do lema 3.1.3 aplicado ao conjunto $\left.\left\{\operatorname{ad}_{X}: X \in \mathfrak{h}\right\}\right)$.

Sabe-se que para $\alpha \in \Delta$ o espaço $\mathfrak{g}_{\alpha}$ tem dimensão um. Para $\alpha, \beta \in \mathfrak{h}^{*}, X \in \mathfrak{g}_{\alpha}$, $Y \in \mathfrak{g}_{\beta}$ e $H \in \mathfrak{h}$ temos:

$$
\mathcal{K}([H, X], Y)+\mathcal{K}(X,[H, Y])=0 \Rightarrow(\alpha+\beta)(H) \mathcal{K}(X, Y)=0 .
$$

Segue daí que $\mathfrak{g}_{\alpha}$ e $\mathfrak{g}_{\beta}$ são $\mathcal{K}$-ortogonais para $\beta \neq-\alpha$. Como $\mathcal{K}$ é não degenerada em $\mathfrak{g}$ segue também que $-\alpha \in \Delta$ sempre que $\alpha \in \Delta$ e que a restrição de $\mathcal{K}$ a $\mathfrak{h}$ é não degenerada.

Temos um isomorfismo $H \mapsto \mathcal{K}(H, \cdot)$ entre $\mathfrak{h}$ e $\mathfrak{h}^{*}$. Para $\alpha \in \mathfrak{h}^{*}$ denotamos por $H_{\alpha}$ o elemento de $\mathfrak{h}$ correspondente por esse isomorfismo. Denotamos por $\mathfrak{h}_{\mathbb{R}}$ o subespaço real de $\mathfrak{h}$ gerado pelos vetores $H_{\alpha} \operatorname{com} \alpha \in \Delta$. Sabe-se que $\mathfrak{h}_{\mathbb{R}}$ é uma forma real de $\mathfrak{h}$ e que a restrição de $\mathcal{K}$ a $\mathfrak{h}_{\mathbb{R}}$ é definida positiva. Além do mais, as raízes de $\mathfrak{g}$ assumem valores reais em $\mathfrak{h}_{\mathbb{R}}$. Observe em particular que os vetores $H_{\alpha}$ com $\alpha \in \Delta$ geram $\mathfrak{h}$ como subespaço complexo (demonstrando diretamente, um vetor não nulo de $\mathfrak{h}$ que fosse $\mathcal{K}$-ortogonal a $H_{\alpha}$ para todo $\alpha \in \Delta$ estaria em $\bigcap_{\alpha \in \Delta} \operatorname{Ker} \alpha$ e portanto no centro de $\mathfrak{g}$ ).

Observe que se $H \in \mathfrak{h}$ é tal que $\alpha(H) \in \mathbb{R}$ para todo $\alpha \in \Delta$ então $H \in \mathfrak{h}_{\mathbb{R}}$. De fato, escrevemos $H=H_{1}+i H_{2}$ com $H_{1}, H_{2} \in \mathfrak{h}_{\mathbb{R}}$. Daí para $\alpha \in \Delta$ temos $\alpha(H) \in \mathbb{R}$ e como $\alpha\left(H_{1}\right), \alpha\left(H_{2}\right) \in \mathbb{R}$ vem $\alpha\left(H_{2}\right)=0$. Como $\alpha \in \Delta$ é arbitrário temos $H_{2}=0$ (pois $H_{2}$ está no centro de $\mathfrak{g}$ ). Segue em particular que $\mathfrak{h}_{\mathbb{R}}$ é a única forma real de $\mathfrak{h}$ onde as raízes assumem valores reais.

Segue da identidade de Jacobi que se $X \in \mathfrak{g}_{\alpha}$ e $Y \in \mathfrak{g}_{\beta}$ então $[X, Y] \in \mathfrak{g}_{\alpha+\beta}$, ou abreviadamente, $\left[\mathfrak{g}_{\alpha}, \mathfrak{g}_{\beta}\right] \subset \mathfrak{g}_{\alpha+\beta}$. Observe em particular que se $\alpha, \beta \in \Delta$ e $\alpha+\beta \notin \Delta \cup\{0\}$ então $\left[\mathfrak{g}_{\alpha}, \mathfrak{g}_{\beta}\right]=0$. Se $\alpha \in \Delta, X \in \mathfrak{g}_{\alpha}$ e $Y \in \mathfrak{g}_{-\alpha}$ então $[X, Y] \in \mathfrak{h}$. Mais precisamente, para todo $H \in \mathfrak{h}$ :

$$
\mathcal{K}([X, Y], H)=\mathcal{K}(Y,[H, X])=\mathcal{K}(X, Y) \alpha(H) .
$$

Segue que $\left[\mathfrak{g}_{\alpha}, \mathfrak{g}_{-\alpha}\right] \subset \mathbb{C} H_{\alpha}$, onde $\mathbb{C} H_{\alpha}$ é o subespaço (complexo) gerado pelo vetor $H_{\alpha}$ (mais precisamente, segue que $\left.[X, Y]=\mathcal{K}(X, Y) H_{\alpha}\right)$.

Observação 3.2.1. Se $\alpha, \beta \in \Delta$ e $\alpha+\beta \in \Delta \cup\{0\}$ então $[X, Y] \neq 0$ sempre que $X \in \mathfrak{g}_{\alpha}$ e $Y \in \mathfrak{g}_{\beta} \operatorname{com} X \neq 0, Y \neq 0$. O caso $\alpha+\beta=0$ segue do que foi dito acima. $\mathrm{O}$ caso $\alpha+\beta \in \Delta$ é muitas vezes enunciado nos textos sob a forma $\left[\mathfrak{g}_{\alpha}, \mathfrak{g}_{\beta}\right]=\mathfrak{g}_{\alpha+\beta}$.

Para finalizar, observamos que é fácil descrever o centralizador de um subconjunto de $\mathfrak{h}$ em termos da decomposição de Cartan. De fato, Se $S \subset \mathfrak{h}$ é um subconjunto então é fácil ver que o centralizador de $S$ é dado por:

$$
\mathfrak{z}(S)=\mathfrak{h} \oplus \bigoplus_{\alpha \in \Delta^{\prime}} \mathfrak{g}_{\alpha}
$$

onde $\Delta^{\prime}$ é o conjunto das raízes $\alpha \in \Delta$ tais que $\alpha_{\mid S}=0$. 


\subsection{Estrutura de álgebras de Lie involutivas ortogonais.}

Seja $\mathfrak{g}$ uma álgebra de Lie real. Seja $s$ um automorfismo involutivo de $\mathfrak{g}$, i.e., $s \in \operatorname{Aut}(\mathfrak{g})$ e $s \circ s=\mathrm{Id}$. Como o polinômio $X^{2}-1 \in \mathbb{R}[X]$ anula $s$ segue que $s$ é diagonalizável com autovalores $1 \mathrm{e}-1$. Escrevemos então $\mathfrak{g}=\mathfrak{g}_{+} \oplus \mathfrak{g}_{-}$, onde $s_{\mid \mathfrak{g}_{+}}=$Id e $s_{\mid \mathfrak{g}_{-}}=-$Id. É fácil ver que $\left[\mathfrak{g}_{+}, \mathfrak{g}_{+}\right] \subset \mathfrak{g}_{+},\left[\mathfrak{g}_{+}, \mathfrak{g}_{-}\right] \subset \mathfrak{g}_{-}$e $\left[\mathfrak{g}_{-}, \mathfrak{g}_{-}\right] \subset \mathfrak{g}_{+}$. Em particular $\mathfrak{g}_{+}$é uma subálgebra de $\mathfrak{g}$ e temos uma representação de $\mathfrak{g}_{+}$no espaço vetorial $\mathfrak{g}_{-}$dada por $X \in \mathfrak{g}_{+} \mapsto\left(\operatorname{ad}_{X}\right)_{\mid \mathfrak{g}_{-}}$. Chamaremos a essa a representação adjunta de $\mathfrak{g}_{+}$em $\mathfrak{g}_{-}$.

Uma álgebra de Lie involutiva ortogonal (ALIO) é uma trinca $(\mathfrak{g}, s, B)$, onde $\mathfrak{g}$ é uma álgebra de Lie real, $s$ é um automorfismo involutivo de $\mathfrak{g}, B$ é um produto interno em $\mathfrak{g}_{-}$ invariante pela representação adjunta de $\mathfrak{g}_{+}$e supomos também que $\mathfrak{g}_{+}$não contém ideais não nulos de $\mathfrak{g}$. A invariância de $B$ significa que $\left(\operatorname{ad}_{X}\right)_{\mathfrak{g}_{-}}$é $B$-antisimétrico para $X \in \mathfrak{g}_{+}$ ou mais explicitamente:

$$
B([X, Y], Z)+B(Y,[X, Z])=0
$$

para $X \in \mathfrak{g}_{+}$e $Y, Z \in \mathfrak{g}_{-}$.

O conceito de ALIO aparece naturalmente na teoria dos espaços Riemannianos simétricos, porém suas propriedades algébricas podem ser estudadas de maneira independente. Em certo sentido, a relação entre ALIOs e espaços Riemannianos simétricos é semelhante à relação entre álgebras de Lie e grupos de Lie. Explicamos rapidamente a construção de uma ALIO a partir de um espaço Riemanniano simétrico a título de motivação, embora isso não seja relevante para o resto do texto.

Um espaço Riemanniano simétrico é uma variedade Riemanniana conexa $M$ tal que para todo $x \in M$ existe uma isometria $\psi: M \rightarrow M \operatorname{com} \psi(x)=x$ e $d \psi_{x}=-$ Id.

Seja $G$ o grupo das isometrias de $M$ (com a topologia compacto-aberta). Sabe-se que $G$ é um grupo de Lie e a ação canônica $G \times M \rightarrow M$ é diferenciável. É possível mostrar que todo espaço simétrico é homogêneo, i.e., a ação de $G$ é transitiva. Fixado então $x_{0} \in M$, podemos identificar $M \operatorname{com} G / G_{+}$, onde $G_{+}$é o subgrupo de isotropia de $x_{0}$. Seja $\psi$ a (única) isometria de $M \operatorname{com} \psi\left(x_{0}\right)=x_{0}$ e $d \psi_{x_{0}}=-$ Id. Daí $\psi^{2}$ é uma isometria de $M$ que fixa $x_{0}$ e cuja diferencial no ponto $x_{0}$ é a identidade. Segue que $\psi^{2}=$ Id e o automorfismo interno $\mathcal{I}_{\psi}$ de $G$ é involutivo, i.e., seu quadrado é a identidade. Seja $\mathfrak{g}$ a álgebra de Lie de $G$ e $s=\operatorname{Ad}_{\psi}=d \mathcal{I}_{\psi}(1)$. Daí g é uma álgebra de Lie (real) e $s$ é um automorfismo involutivo de $\mathfrak{g}$. Escrevemos $\mathfrak{g}=\mathfrak{g}_{+} \oplus \mathfrak{g}_{-}$como antes e não é difícil ver que $\mathfrak{g}_{+}$é de fato a álgebra de Lie de $G_{+}$. Podemos então identificar $T_{x_{0}} M$ com $\mathfrak{g} / \mathfrak{g}_{+} \cong \mathfrak{g}_{-}$. O produto interno em $T_{x_{0}} M$ induz então um produto interno $B$ em $\mathfrak{g}_{-}$, cuja invariância pela representação adjunta de $\mathfrak{g}_{+}$segue da $G$-invariânvia da métrica Riemanniana de $M$. A inexistência de ideais não nulos de $\mathfrak{g}$ contidos em $\mathfrak{g}_{+}$segue da efetividade da ação de $G$ em $M$ (vide lema 3.3.3). A homogeneidade de $M$ implica que a (classe de isomorfismo da) ALIO (g, $s, B)$ construída não depende da escolha de $x_{0} \in M$.

Faremos a partir de agora uma exposição de alguns resultados algébricos sobre ALIOs. Seja então $(\mathfrak{g}, s, B)$ uma ALIO. Vamos estabelecer algumas relações entre os espaços $\mathfrak{g}_{+}$ e $\mathfrak{g}_{-}$e a forma de Killing $\mathcal{K}$. Em primeiro lugar, como $s$ é $\mathcal{K}$-ortogonal, segue facilmente que $\mathfrak{g}_{+}$e $\mathfrak{g}_{-}$são $\mathcal{K}$-ortogonais, i.e., $\mathcal{K}(X, Y)=0$ para $X \in \mathfrak{g}_{+}$e $Y \in \mathfrak{g}_{-}$. Basta ver que:

$$
\mathcal{K}(X, Y)=\mathcal{K}(s(X), s(Y))=\mathcal{K}(X,-Y)
$$


Mostraremos a seguir que a restrição de $\mathcal{K}$ a $\mathfrak{g}_{+}$é definida negativa. Antes temos um lema.

Lema 3.3.1. A representação adjunta de $\mathfrak{g}_{+}$em $\mathfrak{g}_{-}$é efetiva, i.e., se $X \in \mathfrak{g}_{+} e\left(\operatorname{ad}_{X}\right)_{\mid \mathfrak{g}_{-}}=$ 0 então $X=0$.

Demonstração. Temos que mostrar que o centralizador $\mathfrak{z}_{\mathfrak{g}_{+}}\left(\mathfrak{g}_{-}\right)$de $\mathfrak{g}_{-}$em $\mathfrak{g}_{+}$é zero. Basta ver que o mesmo é um ideal de $\mathfrak{g}$. Seja então $X \in \mathfrak{z}_{\mathfrak{g}_{+}}\left(\mathfrak{g}_{-}\right)$. Se $Y \in \mathfrak{g}_{+}$, segue facilmente da identidade de Jacobi que $[X, Y] \in \mathfrak{z}_{\mathfrak{g}_{+}}\left(\mathfrak{g}_{-}\right)$e se $Y \in \mathfrak{g}_{-}$temos $[X, Y]=0$.

Observação 3.3.1. Vale também uma espécie de recíproca do lema acima. Sejam g uma álgebra de Lie e $s$ um automorfismo involutivo de $\mathfrak{g}$. Escrevemos como sempre $\mathfrak{g}=\mathfrak{g}_{+} \oplus \mathfrak{g}_{-}$, onde $s_{\mid \mathfrak{g}_{+}}=$Id e $s_{\mid \mathfrak{g}_{-}}=-$Id. Se a representação adjunta de $\mathfrak{g}_{+}$em $\mathfrak{g}_{-}$é efetiva, i.e., se nenhum elemento não nulo de $\mathfrak{g}_{+}$centraliza $\mathfrak{g}_{-}$então $\mathfrak{g}_{+}$não contém ideais não nulos de g. De fato, se $\mathfrak{i} \subset \mathfrak{g}_{+}$é um ideal de $\mathfrak{g}$ então, por um lado $\left[\mathfrak{i}, \mathfrak{g}_{-}\right] \subset \mathfrak{i} \subset \mathfrak{g}_{+}$(pois i é um ideal) e por outro lado $\left[\mathfrak{i}, \mathfrak{g}_{-}\right] \subset \mathfrak{g}_{-}$, donde $\left[\mathfrak{i}, \mathfrak{g}_{-}\right]=0$.

Corolário. A álgebra de Lie $\mathfrak{g}_{+}$admite um produto interno invariante pela sua própria representação adjunta, i.e., tal que $\left(\operatorname{ad}_{X}\right)_{\left.\right|_{\mathfrak{g}_{+}}}$é antisimétrico para todo $X \in \mathfrak{g}_{+}$.

Demonstração. Considere o grupo de Lie compacto $\mathrm{SO}\left(\mathfrak{g}_{-}\right)$das transformações $B$-ortogonais positivas de $\mathfrak{g}_{-}$. Sua álgebra de Lie so( $\left.\mathfrak{g}_{-}\right)$das transformações $B$-antisimétricas de $\mathfrak{g}_{-}$ admite um produto interno invariante pela representação adjunta de $\mathrm{SO}\left(\mathfrak{g}_{-}\right)$e portanto invariante pela sua própria representação adjunta. A efetividade da representação adjunta de $\mathfrak{g}_{+}$em $\mathfrak{g}_{-}$nos mostra que $\mathfrak{g}_{+}$é (isomorfa a) uma subálgebra de so(g $\mathfrak{g}_{-}$). Basta então restringir o produto interno de $\mathrm{so}\left(\mathfrak{g}_{-}\right)$a $\mathfrak{g}_{+}$.

O corolário acima pode também ser enunciado dizendo que $\mathfrak{g}_{+}$é uma álgebra de Lie compacta.

Lema 3.3.2. A restrição da forma de Killing de $\mathfrak{g}$ a $\mathfrak{g}_{+}$é definida negativa.

Demonstração. Pelo corolário do lema 3.3.1, podemos estender o produto interno $B$ de $\mathfrak{g}_{-}$ a $\mathfrak{g}$, declarando que sua restrição a $\mathfrak{g}_{+}$seja invariante pela representação adjunta de $\mathfrak{g}_{+} \mathrm{e}$ que $\mathfrak{g}_{+}$e $\mathfrak{g}_{-}$sejam ortogonais. Obtemos então um produto interno invariante pela representação adjunta de $\mathfrak{g}_{+}$em $\mathfrak{g}$. Em particular, para $X \in \mathfrak{g}_{+}$temos que $\operatorname{ad}_{X}$ é representado por uma matriz antisimétrica em bases ortonormais e portanto $\operatorname{tr}\left(\operatorname{ad}_{X}\right)^{2} \leq 0$, valendo a igualdade se e somente se $\operatorname{ad}_{X}=0 . \operatorname{Mas}_{X}=0$ implica $X=0$ pelo lema 3.3.1.

Observação 3.3.2. O produto interno cuja existência é demonstrada no corolário do lema 3.3.1 pode ser tomado como a restrição de $-\mathcal{K}$ a $\mathfrak{g}_{+}$, pelo lema acima (note porém que na demonstração acima o referido corolário é utilizado, por isso o mesmo precisou de uma demonstração independente). Observe que a restrição a $\mathfrak{g}_{+}$da forma de Killing de $\mathfrak{g}$ em geral não coincide com a forma de Killing de $\mathfrak{g}_{+}$, e na verdade a última pode ser degenerada (isso ocorre quando $\mathfrak{g}_{+}$possui centro).

Nosso próximo lema não é puramente algébrico e é independente do desenvolvimento da teoria de ALIOs, porém fornece um critério útil para determinar se uma trinca $(\mathfrak{g}, s, B)$ é uma ALIO quando $g$ é dada como álgebra de Lie de um grupo de Lie $G$. 
Lema 3.3.3. Seja dada uma ação transitiva de um grupo de Lie conexo $G$ numa variedade $M$. Seja $m \in M$ um ponto com grupo de isotropia $K$. Sejam $\mathfrak{g}$ e $\mathfrak{k}$ as álgebras de Lie dos grupos $G$ e $K$ respectivamente. Suponha que a ação de $G$ em $M$ é efetiva, i.e., se $g \cdot x=x$ para todo $x \in M$ então $g=1$. Então k não contém ideais não nulos de $\mathfrak{g}$.

Demonstração. Se existisse um ideal não nulo $\mathfrak{n}$ de $\mathfrak{g}$ com $\mathfrak{n} \subset \mathfrak{k}$, teríamos um subgrupo de Lie conexo $N \subset G$ não trivial associado à álgebra de Lie n. Como $N$ e $G$ são conexos segue que $N$ é normal em $G$ (n é invariante pela representação adjunta de g e portanto pela representação adjunta de $G$, pois $G$ é conexo. Como $N$ é conexo segue que $N$ é invariante pelos automorfismos internos de $G$ ). Como $\mathfrak{n} \subset \mathfrak{k}$ e $N$ é conexo segue $N \subset K$. Afirmamos que $N$ age trivialmente em $M$.

De fato, sejam $g \in N, x \in M$. Como a ação de $G$ é transitiva escrevemos $x=h \cdot m$ para algum $h \in G$. Daí:

$$
g \cdot x=(g h) \cdot m=h \cdot\left(\left(h^{-1} g h\right) \cdot m\right)=h \cdot m=x,
$$

pois $h^{-1} g h \in N \subset K$. Chegamos a uma contradição.

O seguinte lema técnico será necessário posteriormente.

Lema 3.3.4. Sejam $V, W$ dois subespaços $\mathcal{K}$-ortogonais de $\mathfrak{g}_{-}$. Suponha que $\left[\mathfrak{g}_{+}, V\right] \subset V$. Então $[V, W]=0$.

Demonstração. Sejam $X \in V, Y \in W$. Como $Z=[X, Y] \in \mathfrak{g}_{+}$, basta mostrar que $\mathcal{K}(Z, Z)=0$, pelo lema 3.3.2. Mas:

$$
\mathcal{K}(Z, Z)=\mathcal{K}([X, Y], Z)=\mathcal{K}(Y,[Z, X])=0
$$

pois $Y \in W$ e $[Z, X] \in V$.

Uma observação simples mas importante é que um subespaço $s$-invariante $V \subset \mathfrak{g}$, i.e., $s(V) \subset V$ se decompõe $V=\left(V \cap \mathfrak{g}_{+}\right) \oplus\left(V \cap \mathfrak{g}_{-}\right)$. Isso segue do fato que $s_{\mid V}$ continua diagonalizável. Em particular, o centro $\mathfrak{z}(\mathfrak{g})$ se decompõe dessa maneira e como $\mathfrak{z}(\mathfrak{g}) \cap \mathfrak{g}_{+}$ é um ideal vemos que $\mathfrak{z}(\mathfrak{g}) \subset \mathfrak{g}_{-}$. O lema abaixo nos dá uma maneira simples de verificar se uma ALIO é semi-simples.

Lema 3.3.5. O centralizador $\mathfrak{z}_{\left(\mathfrak{g}_{-}\right)}$de $\mathfrak{g}_{-}$é um ideal abeliano de $\mathfrak{g}$ contido em $\mathfrak{g}_{-}$. Além do mais, $\mathfrak{z}\left(\mathfrak{g}_{-}\right)=0$ se e somente se $\mathfrak{g}$ é semi-simples.

Demonstração. É fácil ver que $\mathfrak{z}_{\left(\mathfrak{g}_{-}\right)}$é $s$-invariante e portanto se escreve como soma direta de sua interseção com $\mathfrak{g}_{+}$e de sua interseção com $\mathfrak{g}_{-}$. Segue então do lema 3.3 .1 que

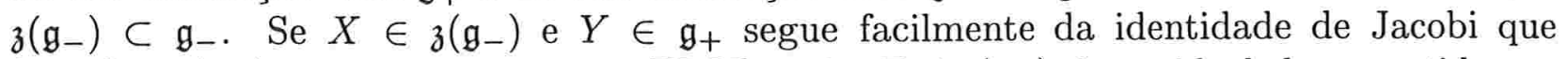
$[X, Y] \in \mathfrak{z}\left(\mathfrak{g}_{-}\right)$e se $Y \in \mathfrak{g}_{-}$temos $[X, Y]=0$. Daí $\mathfrak{z}\left(\mathfrak{g}_{-}\right)$é um ideal de $\mathfrak{g}$ contido em $\mathfrak{g}_{-}$. Como $\left[\mathfrak{g}_{-}, \mathfrak{g}_{-}\right] \subset \mathfrak{g}_{+}$temos que toda subálgebra contida em $\mathfrak{g}_{-}$é abeliana, donde em particular $\mathfrak{z}\left(\mathfrak{g}_{-}\right)$é abeliano.

Se $\mathfrak{g}$ é semi-simples então $\mathfrak{g}$ não possui ideais abelianos não nulos e portanto $\mathfrak{z}\left(\mathfrak{g}_{-}\right)=0$. Suponha agora que $\mathfrak{g}$ não é semi-simples e mostremos que $\mathfrak{z}\left(\mathfrak{g}_{-}\right) \neq 0$. Seja $\mathfrak{n} \subset \mathfrak{g}$ o núcleo da forma de Killing $\mathcal{K}$. Como $\mathfrak{n}$ é $s$-invariante segue que $\mathfrak{n}=\left(\mathfrak{n} \cap \mathfrak{g}_{+}\right) \oplus\left(\mathfrak{n} \cap \mathfrak{g}_{-}\right)$. Se fosse $\mathfrak{n} \cap \mathfrak{g}_{-}=0$ então $\mathfrak{n}$ seria um ideal não nulo de $\mathfrak{g}$ contido em $\mathfrak{g}_{+}$, donde $\mathfrak{n} \cap \mathfrak{g}_{-} \neq 0$. 
Aplicando o lema 3.3.4 com $V=\mathfrak{g}_{-}$e $W=\mathfrak{n} \cap \mathfrak{g}_{-}$vem $[V, W]=0$ e $\operatorname{logo} W \subset \mathfrak{z}\left(\mathfrak{g}_{-}\right) \neq 0$.

Uma ALIO g é dita de tipo compacto quando a restrição de sua forma de Killing $\mathcal{K}$ a $\mathfrak{g}_{-}$ for definida negativa (segue então do lema 3.3.2 que $\mathcal{K}$ é definida negativa em $\mathfrak{g}$ ). Diremos que $\mathfrak{g}$ é de tipo não-compacto quando a restrição de $\mathcal{K}$ a $\mathfrak{g}$ - for definida positiva. Note que em ambos os casos $\mathcal{K}$ é não degenerada e portanto g é semi-simples. Uma ALIO g é dita euclideana quando a restrição de $\mathcal{K}$ a $\mathfrak{g}_{-}$for nula. Isso equivale a dizer que $\mathfrak{g}_{-}$é abeliana. De fato, se $\mathcal{K}$ se anula em $\mathfrak{g}_{-}$, segue do lema 3.3 .4 que $\left[\mathfrak{g}_{-}, \mathfrak{g}_{-}\right]=0$. Reciprocamente, se $\mathfrak{g}_{-}$é abeliana então na verdade $\mathfrak{g}_{-}$é um ideal abeliano de $\mathfrak{g}$ e logo $\mathcal{K}$ se anula em $\mathfrak{g}_{-}$. Note que uma ALIO euclideana $\mathfrak{g}$ só pode ser semi-simples se $\mathfrak{g}=0$. De fato, nesse caso $\mathfrak{g}_{-}$ é um ideal abeliano e portanto $\mathfrak{g}_{-}=0$. Daí $\mathfrak{g}_{+}=\mathfrak{g}$ é um ideal de $\mathfrak{g}$ e portanto também $\mathrm{g}_{+}=0$.

Se $\mathfrak{g} \neq 0$, as possibilidades explicadas acima são mutuamente exclusivas, porém não exaustivas. Dizemos que uma ALIO g é irredutivel quando não for euclideana e a representação adjunta de $\mathfrak{g}_{+}$em $\mathfrak{g}_{-}$for irredutível. Isso significa que se $V$ é um subespaço de $\mathfrak{g}_{-} \operatorname{com}\left[\mathfrak{g}_{+}, V\right] \subset V$ então $V=0$ ou $V=\mathfrak{g}_{-}$. Temos então o seguinte lema.

Lema 3.3.6. Se g é uma ALIO irredutível então g é de tipo compacto ou de tipo nãocompacto.

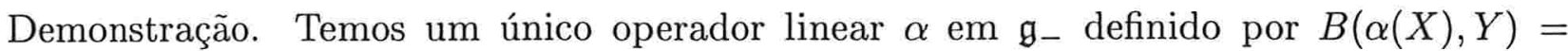
$\mathcal{K}(X, Y)$, para $X, Y \in \mathfrak{g}_{-}$. Daí $\alpha$ é $B$-simétrico e portanto possui um autovalor real $c \in \mathbb{R}$. É fácil ver que $\alpha$ comuta com $\operatorname{ad}_{Z}$ para todo $Z \in \mathfrak{g}_{+}$, donde o autoespaço $V=\operatorname{Ker}(\alpha-c \mathrm{Id})$ satisfaz $\left[\mathfrak{g}_{+}, V\right] \subset V$. Como $V \neq 0$ temos $V=\mathfrak{g}_{-}$e $\operatorname{logo} \mathcal{K}=c B$ em $\mathfrak{g}_{-}$. Não pode ser $c=0$ pois g não é euclideana. Se $c>0$ temos que $\mathfrak{g}$ é de tipo não-compacto e se $c<0$ temos que $\mathfrak{g}$ é de tipo compacto.

Corolário. Toda ALIO irredutível é semi-simples.

Demonstração. Segue diretamente do lema e dos comentário que o precedem.

O próximo lema nos diz agora que toda ALIO se escreve como soma direta de uma componente euclideana e de componentes irredutíveis.

Lema 3.3.7. Seja $(\mathfrak{g}, s, B)$ uma ALIO. Então podemos escrever $\mathfrak{g}=\mathfrak{g}^{0} \oplus \cdots \oplus \mathfrak{g}^{n}$ como soma direta de ideais $s$-invariantes, de modo que $\left(\mathfrak{g}^{0}, s^{0}, B^{0}\right)$ é uma ALIO euclideana e $\left(\mathfrak{g}^{i}, s^{i}, B^{i}\right)$ é uma ALIO irredutível para $i=1, \ldots, n$, onde $s^{i}$ é a restrição de $s$ a $\mathfrak{g}^{i}$ e $B^{i}$ é a restrição de $B$ a $\mathfrak{g}_{-} \cap \mathfrak{g}^{i}$. Além do mais, $\mathfrak{g}$ é semi-simples se e somente se $\mathfrak{g}^{0}=0$.

Demonstração. Defina um operador linear $\alpha$ em $\mathfrak{g}_{\text {- }}$ como na demonstração do lema 3.3.6. Temos novamente que $\alpha$ é $B$-simétrico e portanto podemos escrever $\mathfrak{g}_{-}=\mathfrak{g}_{-}^{0} \oplus \cdots \oplus \mathfrak{g}_{-}^{n}$, onde $\mathfrak{g}_{-}^{i}$ é o autoespaço de $\alpha$ associado ao autovalor $c_{i} \in \mathbb{R}$ e $\mathfrak{g}_{-}^{i}$ é $B$-ortogonal a $\mathfrak{g}_{-}^{j}$ para $i \neq j$. Supomos $c_{0}=0$ e fazemos $\mathfrak{g}_{-}^{0}=0$ se 0 não for autovalor de $\alpha$ (supomos também que $c_{i} \neq 0$ para $\left.i=1, \ldots, n\right)$. Observe que se $X \in \mathfrak{g}_{-}^{i}$ e $Y \in \mathfrak{g}_{-}^{j} \operatorname{com} i \neq j$ então:

$$
\mathcal{K}(X, Y)=B(\alpha(X), Y)=c_{i} B(X, Y)=0
$$

de modo que os subespaços $\mathfrak{g}_{-}^{i}$ são também $\mathcal{K}$-ortogonais. 
Como na demonstração do lema 3.3 .6 concluímos que $\left[\mathfrak{g}_{+}, \mathfrak{g}_{-}^{i}\right] \subset \mathfrak{g}_{-}^{i}$, pois $\mathfrak{g}_{-}^{i}$ é um autoespaço de $\alpha$. A representação adjunta de $\mathfrak{g}_{+}$em $\mathfrak{g}_{-}^{i}$ em geral não é irredutível, mas podemos escrever $\mathfrak{g}_{-}^{i}$ como soma direta de subespaços irredutíveis. De fato, apenas observe que se um subespaço $V \subset \mathfrak{g}_{-}^{i}$ satisfaz $\left[\mathfrak{g}_{+}, V\right] \subset V$ então o complemento $B$-ortogonal $V^{\perp}$ de $V$ em $\mathfrak{g}_{-}^{i}$ também satisfaz $\left[\mathfrak{g}_{+}, V^{\perp}\right] \subset V^{\perp}$. Observe que podemos supor que esses subespaços irredutíveis são $B$-ortogonais e como $\mathcal{K}=c_{i} B$ em $\mathfrak{g}_{-}^{i}$, vemos que os mesmos serão também $\mathcal{K}$-ortogonais.

Resumindo a construção acima e renomeando os subespaços $\mathfrak{g}_{-}^{i}$ se necessário, podemos escrever $\mathfrak{g}_{-}=\mathfrak{g}_{-}^{0} \oplus \cdots \oplus \mathfrak{g}_{-}^{n}$, onde $\mathfrak{g}_{-}^{i}$ e $\mathfrak{g}_{-}^{j}$ são $B$-ortogonais e $\mathcal{K}$-ortogonais para $i \neq j$, $\left[\mathfrak{g}_{+}, \mathfrak{g}_{-}^{i}\right] \subset \mathfrak{g}_{-}^{i}, \mathfrak{g}_{-}^{0}$ é o núcleo de $\alpha$, a restrição de $\alpha$ a $\mathfrak{g}_{-}^{i}$ é um múltiplo não nulo da identidade para $i \geq 1$ e a representação adjunta de $\mathfrak{g}_{+}$em $\mathfrak{g}_{-}^{i}$ é irredutível para $i \geq 1$. Segue do lema 3.3 .4 que $\left[\mathfrak{g}_{-}^{i}, \mathfrak{g}_{-}^{j}\right]=0$ para $i \neq j$.

Para $i=1, \ldots, n$ seja $\mathfrak{g}_{+}^{i}$ o subespaço gerado pelos colchetes $[X, Y] \operatorname{com} X, Y \in \mathfrak{g}_{-}^{i}$. Note que $\mathfrak{g}_{+}^{i} \subset \mathfrak{g}_{+}$. Escreva $\mathfrak{g}^{i}=\mathfrak{g}_{+}^{i} \oplus \mathfrak{g}_{-}^{i} \subset \mathfrak{g}$. Mostraremos que $\mathfrak{g}^{i}$ é um ideal de $\mathfrak{g}$. Temos $\left[\mathfrak{g}_{+}, \mathfrak{g}_{-}^{i}\right] \subset \mathfrak{g}_{-}^{i}$ e $\left[\mathfrak{g}_{-}, \mathfrak{g}_{-}^{i}\right]=\left[\mathfrak{g}_{-}^{i}, \mathfrak{g}_{-}^{i}\right] \subset \mathfrak{g}_{+}^{i}$. Segue da identidade de Jacobi que $\left[\mathfrak{g}_{+}, \mathfrak{g}_{+}^{i}\right] \subset \mathfrak{g}_{+}^{i}$ e que $\left[\mathfrak{g}_{-}, \mathfrak{g}_{+}^{i}\right] \subset \mathfrak{g}_{-}^{i}$, se observarmos que $\left[\mathfrak{g}_{-}, \mathfrak{g}_{-}^{i}\right] \subset \mathfrak{g}_{+}$.

Mostramos então que $\mathfrak{g}^{i}$ é um ideal de $\mathfrak{g}$ e é claro que $s\left(\mathfrak{g}^{i}\right)=\mathfrak{g}^{i}$, donde podemos considerar a restrição $s^{i}$ de $s$ a $\mathfrak{g}^{i}$, a qual é obviamente um automorfismo involutivo da álgebra de Lie (real) $\mathfrak{g}^{i}$. Temos que $\mathfrak{g}^{i}=\mathfrak{g}_{+}^{i} \oplus \mathfrak{g}_{-}^{i}$ é a decomposição de $\mathfrak{g}^{i}$ em autoespaços de $s^{i}$. Denotando por $B^{i}$ a restrição de $B$ a $\mathfrak{g}_{-}^{i}$, é claro que $B^{i}$ é um produto interno invariante pela representação adjunta de $\mathfrak{g}_{+}^{i}$ em $\mathfrak{g}_{-}^{i}$.

Sejam $X, Y \in \mathfrak{g}_{-}^{i}$ e $Z \in \mathfrak{g}_{+}^{j} \operatorname{com} i, j=1, \ldots, n, i \neq j$. Segue da identidade de Jacobi que $\left[\mathfrak{g}_{-}^{i}, \mathfrak{g}_{+}^{j}\right]=0$. Temos então:

$$
\mathcal{K}([X, Y], Z)=\mathcal{K}(Y,[Z, X])=0 .
$$

Segue que $\mathfrak{g}_{+}^{i}$ e $\mathfrak{g}_{+}^{j}$ são $\mathcal{K}$-ortogonais. Pelo lema $3.3 .2, \mathcal{K}$ é definida negativa em $\mathfrak{g}_{+}$, donde a soma $\mathfrak{g}_{+}^{1} \oplus \cdots \oplus \mathfrak{g}_{+}^{n}$ é direta. Segue finalmente que a soma $\mathfrak{g}^{\prime}=\mathfrak{g}^{1} \oplus \cdots \oplus \mathfrak{g}^{n}$ é direta. Note que $\mathcal{K}$ é não degenerada em $\mathfrak{g}^{\prime}$. De fato, os espaços $\mathfrak{g}_{+}^{i}$ e $\mathfrak{g}_{-}^{j}$ são todos $\mathcal{K}$-ortogonais entre si, sendo que $\mathcal{K}$ é definida negativa em $\mathfrak{g}_{+}^{i}$ e definida positiva ou negativa em $\mathfrak{g}_{-}^{j}$ (recorde que $\mathcal{K}$ é um múltiplo não nulo de $B$ em $\mathfrak{g}_{-}^{j}$ ). Denotando por $\mathfrak{g}^{0}$ o complemento $\mathcal{K}$-ortogonal de $\mathfrak{g}^{\prime}$ em $\mathfrak{g}$, segue então do lema 3.1.1 que $\mathfrak{g}=\mathfrak{g}^{0} \oplus \mathfrak{g}^{\prime}$.

Como $\mathfrak{g}^{\prime}$ é um ideal $s$-invariante, segue que $\mathfrak{g}^{0}$ também o é. É fácil ver que $\mathfrak{g}_{-} \cap \mathfrak{g}^{0}$ coincide com o espaço $\mathfrak{g}_{-}^{0}$ definido anteriormente e podemos agora definir $\mathfrak{g}_{+}^{0}=\mathfrak{g}_{+} \cap \mathfrak{g}^{0}$. Daí a restrição $s^{0}$ de $s$ a $\mathfrak{g}^{0}$ é um automorfismo involutivo da álgebra de Lie (real) $\mathfrak{g}^{0}$, $\mathfrak{g}^{0}=\mathfrak{g}_{+}^{0} \oplus \mathfrak{g}_{-}^{0}$ é a decomposição em autoespaços de $s^{0}$ e a restrição $B^{0}$ de $B$ a $\mathfrak{g}_{-}^{0}$ é um produto interno invariante pela representação adjunta de $\mathfrak{g}_{+}^{0}$.

É fácil ver agora que se $\mathfrak{n}$ é um ideal de $\mathfrak{g}^{i}$ então $\mathfrak{n}$ também é um ideal de $\mathfrak{g}$, pois $\left[\mathfrak{g}^{j}, \mathfrak{n}\right]=0$ para $j \neq i$. Segue que $\mathfrak{g}_{+}^{i}$ não contém ideais não nulos de $\mathfrak{g}^{i}$ e portanto as trincas $\left(g^{i}, s^{i}, B^{i}\right)$ são de fato ALIOs. Para $i \geq 1$, sabemos que a representação adjunta de $\mathfrak{g}_{+}$em $\mathfrak{g}_{-}^{i}$ é irredutível, mas segue facilmente agora que sua restrição a $\mathfrak{g}_{+}^{i}$ também o é. Sabemos que $\mathcal{K}$ se anula em $\mathfrak{g}_{-}^{0}$ e não se anula em $\mathfrak{g}_{-}^{i}$ para $i \geq 1$ e (como a forma de Killing de um ideal é simplesmente a restrição da forma de Killing de g), segue de fato que $\mathfrak{g}^{0}$ é uma ALIO euclideana e $\mathfrak{g}^{i}$ é uma ALIO irredutível para $i \geq 1$. 
Finalmente, se $\mathfrak{g}$ é semi-simples então o ideal abeliano $\mathfrak{g}_{-}^{0}$ deve ser nulo, donde $\mathfrak{g}^{0}=0$. Se $\mathfrak{g}^{0}=0$ então todos os ideais $\mathfrak{g}^{i}$ são semi-simples, donde $\mathfrak{g}$ o é.

Queremos agora relacionar a teoria de ALIOs semi-simples com a teoria de estrutura de álgebras de Lie complexas semi-simples. Como já foi observado antes, uma álgebra de Lie real $\mathfrak{g}$ é semi-simples se e somente se sua complexificação $\mathfrak{g}^{c} \mathrm{o}$ for. Temos o seguinte lema.

Lema 3.3.8. Seja $(\mathfrak{g}, s, B)$ uma ALIO com g semi-simples. Sejam a uma subálgebra abeliana maximal de $\mathfrak{g}_{-}$(i.e., a $\subset \mathfrak{g}_{-}$é abeliana e não está propriamente contida em nenhuma subálgebra abeliana de $\left.\mathfrak{g}_{-}\right), \mathfrak{k}=\mathfrak{z}_{\mathfrak{g}_{+}}(\mathfrak{a})$ o centralizador de $\mathfrak{a}$ em $\mathfrak{g}_{+}$e $\mathfrak{t}$ uma subálgebra abeliana maximal de $\mathfrak{k}$. Definindo $\mathfrak{h}=\mathfrak{t} \oplus \mathfrak{a}$ temos que $\mathfrak{h}^{c}$ é uma subálgebra de Cartan da álgebra de Lie complexa semi-simples $\mathfrak{g}^{c}$.

Demonstração. Temos que $t$ e $\mathfrak{a}$ são abelianas e $\mathfrak{t} \subset \mathfrak{k} \operatorname{com}[\mathfrak{k}, \mathfrak{a}]=0$. Segue que $\mathfrak{h}$ é abeliana. Mostremos que $\mathfrak{h}$ é abeliana maximal em $\mathfrak{g}$. Basta mostrar que $\mathfrak{z}(\mathfrak{h}) \subset \mathfrak{h}$. Como $\mathfrak{h}$ é $s$-invariante, segue que $\mathfrak{z}(\mathfrak{h})$ também o é, donde:

$$
\mathfrak{z}(\mathfrak{h})=\left(\mathfrak{z}(\mathfrak{h}) \cap \mathfrak{g}_{+}\right) \oplus\left(\mathfrak{z}(\mathfrak{h}) \cap \mathfrak{g}_{-}\right) .
$$

Mas $\mathfrak{z}(\mathfrak{h}) \cap \mathfrak{g}_{+}$centraliza $\mathfrak{a}$, donde está contido em $\mathfrak{k}$. Como $\mathfrak{z}(\mathfrak{h}) \cap \mathfrak{g}_{+}$também centraliza $t$, vemos que o mesmo está contido em $\mathfrak{t}$. Também $\mathfrak{z}(\mathfrak{h}) \cap \mathfrak{g}$ - centraliza $\mathfrak{a}$, donde está contido em a.

Vemos então que $\mathfrak{h}$ é abeliana maximal em $\mathfrak{g}$ e segue facilmente então que $\mathfrak{h}^{c}$ é abeliana maximal em $\mathfrak{g}^{c}$. Resta mostrar que $\operatorname{ad}_{X}$ é diagonalizável em $\mathfrak{g}^{c}$ para $X \in \mathfrak{h}^{c}$. Usaremos o corolário do lema 3.1.3.

Defina um produto interno em $\mathfrak{g}$ declarando que $\mathfrak{g}_{+}$e $\mathfrak{g}_{-}$sejam ortogonais, que sua restrição a $\mathfrak{g}_{+}$seja $-\mathcal{K}$ e que sua restrição a $\mathfrak{g}_{-}$seja $B$ (segue do lema 3.3 .2 que temos de fato um produto interno). Daí $\operatorname{ad}_{X}$ é antisimétrico em $\mathfrak{g}$ para $X \in \mathfrak{g}_{+}$(e em particular para $X \in \mathfrak{t}$ ). Esse produto interno se estende a um produto hermiteano de $\mathfrak{g}^{c}$ no qual $\operatorname{ad}_{X}$ será anti-hermiteano e portanto diagonalizável (a saber, com autovalores imaginários puros).

Se $\mathfrak{g}$ é de tipo compacto temos que $-\mathcal{K}$ é um produto interno em $\mathfrak{g}$ invariante pela sua própria representação adjunta. Daí $\operatorname{ad}_{X}$ é antisimétrico para todo $X \in \mathfrak{g}$ (e em particular para $X \in \mathfrak{a}$ ) donde fazendo as extensões a $\mathfrak{g}^{c}$ concluímos novamente que $\operatorname{ad}_{X}$ é diagonalizável (com autovalores imaginários puros). Se g é de tipo não-compacto, é fácil ver que a expressão:

$$
\langle X, Y\rangle=-\mathcal{K}(X, s(Y)), \quad X, Y \in \mathfrak{g}
$$

define um produto interno em $\mathfrak{g}$ que torna $\operatorname{ad}_{X}$ simétrica para $X \in \mathfrak{g}_{-}$(e em particular para $X \in \mathfrak{a}$ ), donde $\operatorname{ad}_{X}$ é diagonalizável em $\mathfrak{g}$ e portanto também $\mathrm{em}^{c}$ (com autovalores reais).

Em geral, usamos o lema 3.3.7 para escrever $\mathfrak{g}=\mathfrak{g}^{1} \oplus \cdots \oplus \mathfrak{g}^{n}$ como soma direta de ideais onde cada $\left(\mathfrak{g}^{i}, s^{i}, B^{i}\right)$ é uma ALIO irredutível e portanto de tipo compacto ou não-compacto, pelo lema 3.3.6. Seja $\pi^{i}: \mathfrak{g} \rightarrow \mathfrak{g}^{i}$ a projeção. Escrevendo $\mathfrak{a}^{i}=\pi^{i}(\mathfrak{a})$, temos que $\mathfrak{a}^{1} \oplus \cdots \oplus \mathfrak{a}^{n}$ é uma subálgebra abeliana de $\mathfrak{g}_{-}$que contém $\mathfrak{a}$, donde deve coincidir com a. Observe então que $\mathfrak{a}^{i}=\mathfrak{g}^{i} \cap \mathfrak{a}$. Temos então $\mathfrak{g}^{c}=\left(\mathfrak{g}^{1}\right)^{c} \oplus \cdots \oplus\left(\mathfrak{g}^{n}\right)^{c}$ e se $X \in \mathfrak{a}^{i}$, 
mostramos como antes que $\operatorname{ad}_{X}$ é diagonalizável em $\left(\mathfrak{g}^{i}\right)^{c}$ e como $\operatorname{ad} x$ se anula em $\left(\mathfrak{g}^{j}\right)^{c}$ para $j \neq i$, segue que $\operatorname{ad}_{X}$ é diagonalizável em $\mathfrak{g}^{c}$. Como $\mathfrak{t} \cup \mathfrak{a}^{1} \cup \ldots \cup \mathfrak{a}^{n}$ gera $\mathfrak{h}^{c}$ (sobre $\mathbb{C})$, o resultado segue do corolário do lema 3.1.3.

A partir de agora trabalharemos sempre com a notação e as hipóteses do lema 3.3.8. Podemos agora considerar a decomposição de Cartan de $\mathfrak{g}^{c}$ :

$$
\mathfrak{g}^{c}=\mathfrak{h}^{c} \oplus \bigoplus_{\alpha \in \Delta} \mathfrak{g}_{\alpha}^{c}=\mathfrak{t}^{c} \oplus \mathfrak{a}^{c} \oplus \bigoplus_{\alpha \in \Delta} \mathfrak{g}_{\alpha}^{c}
$$

A demonstração acima nos diz as imagens de $\alpha_{\mid \mathrm{t}}$ e $\alpha_{\mid \mathfrak{a}}$ para $\alpha \in \Delta$.

Corolário (da demonstração). Nas condições do lema, seja $\Delta$ o conjunto das raízes da álgebra de Lie complexa semi-simples $\mathfrak{g}^{c}$ em relação à subálgebra de Cartan $\mathfrak{h}^{c}$. Se $\alpha \in \Delta$ então $\alpha(\mathfrak{t}) \subset i \mathbb{R}$ e $\alpha(\mathfrak{a}) \subset \mathbb{R}$ ou $\alpha(\mathfrak{a}) \subset i \mathbb{R}$.

Demonstração. Se $H \in \mathfrak{h}^{c}$ então $\alpha(H)$ é um autovalor de $\operatorname{ad}_{H}$ (qualquer elemento não nulo de $\mathfrak{g}_{\alpha}^{c}$ é um autovetor). Segue da demonstração do lema que $\alpha(\mathfrak{t}) \subset i \mathbb{R}$ e $\alpha\left(\mathfrak{a}^{j}\right) \subset i \mathbb{R}$ (quando $\mathfrak{g}^{j}$ é de tipo compacto) ou $\alpha\left(\mathfrak{a}^{j}\right) \subset \mathbb{R}$ (quando $\mathfrak{g}^{j}$ é de tipo não-compacto), para $j=1,2, \ldots, n$.

Escrevendo $\mathfrak{h}^{j}=\pi^{j}(\mathfrak{h})$ então $\mathfrak{h}^{1} \oplus \cdots \oplus \mathfrak{h}^{n}$ é uma subálgebra abeliana de $\mathfrak{g}$ que contém $\mathfrak{h}$ e portanto coincide com $\mathfrak{h}$. Observe então que $\mathfrak{h}^{j}=\mathfrak{g}^{j} \cap \mathfrak{h}$. A demonstração ficará completa quando mostrarmos que existe exatamente um índice $j=1,2, \ldots, n$ com $\alpha_{\mid \mathfrak{h}^{j}} \neq 0$.

Como $\alpha \neq 0$, existe um índice $j$ com $\alpha_{\mid \mathfrak{h}^{j}} \neq 0$. Mostremos que $\mathfrak{g}_{\alpha}^{c} \subset\left(\mathfrak{g}^{j}\right)^{c}$. De fato, se $X \in \mathfrak{g}_{\alpha}^{c}$ escolhemos $H \in \mathfrak{h}^{j} \operatorname{com} \alpha(H) \neq 0$. Daí $[H, X]=\alpha(H) X$ pertence a $\left(\mathfrak{g}^{j}\right)^{c}$ (pois $\left(\mathfrak{g}^{j}\right)^{c}$ é um ideal de $\left.\mathfrak{g}^{c}\right)$ e logo $X \in\left(\mathfrak{g}^{j}\right)^{c}$.

Concluímos que $\mathfrak{g}_{\alpha}^{c} \subset\left(\mathfrak{g}^{j}\right)^{c}$ e portanto $\alpha(H)=0$ para $H \in \mathfrak{h}^{k}, k \neq j$.

Observe que o automorfismo $s$ de g se estende a um automorfismo involutivo ( $\mathbb{C}$-linear) $s^{c}$ de $\mathfrak{g}^{c}$ com autoespaços $\mathfrak{g}_{+}^{c}$ e $\mathfrak{g}_{-}^{c}$. Por simplicidade, continuaremos escrevendo $s$ em vez de $s^{c}$. Também escreveremos apenas $\mathcal{K}$ para a forma de Killing $\mathcal{K}^{c}$ de $\mathfrak{g}^{c}$. Para $X \in \mathfrak{g}^{c}$ denotamos por $\bar{X}$ o seu conjugado em relação à forma real $\mathfrak{g}$. Denotaremos por $\mathfrak{h}_{\mathbb{R}}$ a forma real de $\mathfrak{h}^{c}$ gerada pelos vetores $H_{\alpha}$ com $\alpha \in \Delta$. Note que as formas reais $\mathfrak{h}$ e $\mathfrak{h}_{\mathbb{R}}$ de $\mathfrak{h}^{c}$ não coincidem (por exemplo, as raízes de $\mathfrak{g}^{c}$ assumem valores reais em $\mathfrak{h}_{\mathbb{R}}$, mas assumem valores imaginários puros em $t \subset \mathfrak{h}$ ). A conjugação será sempre feita em relação à forma real $\mathfrak{h}$, a menos de menção explícita em contrário.

Recorde que para $\alpha \in \mathfrak{h}^{c *}$ definimos $\bar{\alpha} \in \mathfrak{h}^{c *}$ fazendo $\bar{\alpha}(H)=\overline{\alpha(\bar{H})}$, para $H \in \mathfrak{h}^{c}$. Note também que como $\mathfrak{h}^{c}$ é $s$-invariante podemos considerar $\alpha \circ s \in \mathfrak{h}^{c *}$. Para $H \in \mathfrak{h}^{c}$ e $X \in \mathfrak{g}_{\alpha}^{c}$ temos as identidades:

$$
[H, s(X)]=s[s(H), X]=\alpha(s(H)) s(X) \quad \text { e } \quad[H, \bar{X}]=\overline{[\bar{H}, X]}=\bar{\alpha}(H) \bar{X}
$$

que mostram que $s\left(\mathfrak{g}_{\alpha}^{c}\right) \subset \mathfrak{g}_{\alpha \circ s}^{c}$ e $\overline{g_{\alpha}^{c}} \subset \mathfrak{g}_{\bar{\alpha}}^{c}$. Aplicando essas inclusões para $\alpha \circ s$ e $\bar{\alpha}$ no lugar de $\alpha$, respectivamente, obtemos as inclusões opostas e portanto as igualdades. Temos então que $\alpha \in \Delta$ implica $\bar{\alpha} \in \Delta, \alpha \circ s \in \Delta$ e:

$$
s\left(\mathfrak{g}_{\alpha}^{c}\right)=\mathfrak{g}_{\alpha \circ s}^{c} \text { e } \overline{\mathfrak{g}_{\alpha}^{c}}=\mathfrak{g}_{\bar{\alpha}}^{c} .
$$


Observe agora que para $\alpha \in\left(\mathfrak{h}^{c}\right)^{*}$ temos um vetor $H_{\alpha} \in \mathfrak{h}^{c}$ correspondente pelo isomorfismo $H \mapsto \mathcal{K}(H, \cdot)$. Daí para $H \in \mathfrak{h}^{c}$ temos:

$$
\mathcal{K}\left(s\left(H_{\alpha}\right), H\right)=\mathcal{K}\left(H_{\alpha}, s(H)\right)=\alpha(s(H)) \quad \text { e } \quad \mathcal{K}\left(\bar{H}_{\alpha}, H\right)=\overline{\mathcal{K}\left(H_{\alpha}, \bar{H}\right)}=\bar{\alpha}(H),
$$

donde segue que:

$$
s\left(H_{\alpha}\right)=H_{\alpha \circ s} \quad \text { e } \quad \bar{H}_{\alpha}=H_{\bar{\alpha}} .
$$

Note que $\mathfrak{h}^{c}=\mathfrak{t}^{c} \oplus \mathfrak{a}^{c}$, donde para $\alpha \in\left(\mathfrak{h}^{c}\right)^{*}$ o vetor $H_{\alpha}$ se decompõe de modo único como $H_{\alpha}=T_{\alpha}+A_{\alpha}$, onde $T_{\alpha} \in \mathfrak{t}^{c}$ e $A_{\alpha} \in \mathfrak{a}^{c}$. Segue de (3.3.2) que a forma real $\mathfrak{h}_{\mathbb{R}}$ é $s$-invariante, donde:

$$
\mathfrak{h}_{\mathbb{R}}=\left(\mathfrak{h}_{\mathbb{R}} \cap \mathfrak{g}_{+}^{c}\right) \oplus\left(\mathfrak{h}_{\mathbb{R}} \cap \mathfrak{g}_{-}^{c}\right)
$$

Observação 3.3.3. Segue de (3.3.3) que para $\alpha \in \Delta$ temos $T_{\alpha}, A_{\alpha} \in \mathfrak{h} \mathbb{R}$. Em particular, como $\mathcal{K}$ é definida positiva em $\mathfrak{h}_{\mathbb{R}}$ segue que para $\alpha \in \Delta$ temos $\mathcal{K}\left(A_{\alpha}, A_{\alpha}\right) \geq 0$ (respectivamente, $\mathcal{K}\left(T_{\alpha}, T_{\alpha}\right) \geq 0$ ), valendo a igualdade se e somente se $A_{\alpha}=0$ (respectivamente, $\left.T_{\alpha}=0\right)$.

Temos o seguinte lema descrevendo k em termos da decomposição de Cartan.

Lema 3.3.9. Sob a notação e as hipóteses do lema 3.3.8 temos:

$$
\mathfrak{k}^{c}=\mathfrak{t}^{c} \oplus \bigoplus_{\substack{\alpha \in \Delta \\ \alpha \mid a=0}} \mathfrak{g}_{\alpha}^{c}
$$

Demonstração. Seja $\alpha \in \Delta$ com $\alpha_{\mid \mathfrak{a}}=0$. Mostremos que $\mathfrak{g}_{\alpha}^{c} \subset \mathfrak{g}_{+}^{c}$. De fato, se $\alpha_{\mid \mathfrak{a}}=0$ então $\alpha \circ s=\alpha$ donde $\mathfrak{g}_{\alpha}^{c}$ é $s$-invariante, por (3.3.1). Basta mostrar então que $\mathfrak{g}_{\alpha}^{c} \cap \mathfrak{g}_{-}^{c}=0$. Mas $\mathfrak{g}_{\alpha}^{c}$ está contido no centralizador de $\mathfrak{a}$ donde sua interseção com $\mathfrak{g}_{-}^{c}$ está contida em $\mathfrak{a}^{c}$. Mas $\mathfrak{a}^{c} \subset \mathfrak{h}^{c}$ e $\mathfrak{h}^{c} \cap \mathfrak{g}_{\alpha}^{c}=0$.

Como $\mathfrak{a} \subset \mathfrak{h}^{c}$, segue de (3.2.1) que o centralizador de $\mathfrak{a}$ em $\mathfrak{g}^{c}$ é dado por:

$$
\mathfrak{z}(\mathfrak{a})=\mathfrak{h}^{c} \oplus \bigoplus_{\substack{\alpha \in \Delta \\ \alpha \mid \mathfrak{a}=0}} \mathfrak{g}_{\alpha}^{c}=\mathfrak{t}^{c} \oplus \mathfrak{a}^{c} \oplus \bigoplus_{\substack{\alpha \in \Delta \\ \alpha \mid \mathfrak{a}=0}} \mathfrak{g}_{\alpha}^{c}
$$

onde o primeiro e o terceiro termos estão contidos em $\mathfrak{g}_{+}^{c}$ e o segundo está contido em $\mathfrak{g}_{-}^{c}$. Segue que o primeiro e o terceiro termos formam o centralizador de $\mathfrak{a}$ em $\mathfrak{g}_{+}^{c}$, ou seja, $\mathfrak{k}^{c}$. 


\section{O teorema fundamental de regularidade de curvas minimizantes.}

O objetivo desta seção é o de descrever uma classe de variedades sub-Riemannianas homogêneas (mais precisamente, com distribuição homogênea e métrica arbitrária) nas quais conseguimos demonstrar que toda curva minimizante pca (parametrizada por comprimento de arco) é diferenciável (i.e., de classe $C^{\infty}$ ). Esse objetivo será atingido no teorema 4.4 .

Começamos recordando alguns fatos básicos sobre distribuições involutivas, relacionados ao teorema de Frobenius, que serão necessários na demonstração do teorema fundamental. As demonstrações podem ser encontradas em [Wn].

Sejam $M$ uma variedade e $\mathcal{D}$ uma distribuição em $M$. Dizemos que $\mathcal{D}$ é involutiva quando o colchete de Lie de dois campos vetoriais a valores em $\mathcal{D}$ também toma valores em $\mathcal{D}$. Uma subvariedade integral de $\mathcal{D}$ é uma subvariedade imersa $N \subset M$ (i.e., $N \subset M$ é uma variedade tal que a inclusão é uma imersão, mas que não necessariamente possui a topologia induzida de $M$ ) tal que $T_{n} N=\mathcal{D}_{n}$ para todo $n \in N$. Dizemos que $\mathcal{D}$ é integrável se para todo $m \in M$ existe uma subvariedade integral $N$ de $\mathcal{D}$ com $m \in N$ (na verdade, trocando $N$ por um aberto de $N$, se necessário, podemos supor sempre que $N$ é mergulhada, i.e., possui a topologia induzida de $M$ ).

O teorema de Frobenius nos diz que $\mathcal{D}$ é involutiva se e somente se $\mathcal{D}$ é integrável, donde usaremos os dois termos indistintamente.

Seja $\mathcal{D}$ uma distribuição integrável na variedade $M$ e seja $N \subset M$ uma subvariedade (imersa) integral de $\mathcal{D}$. Temos que uma função a valores em $M$ com imagem contida em $N$ tem sua regularidade preservada quando seu contra-domínio é considerado em $N$ (isso é um fato geral para subvariedades mergulhadas, mas não para subvariedades imersas quaisquer). Mais precisamente, se $f: P \rightarrow M$ é uma função de classe $C^{k}$ (onde $P$ é uma outra variedade qualquer) com $f(P) \subset N$ então $f: P \rightarrow N$ também é de classe $C^{k}$. Também é verdade que se $\gamma:[a, b] \rightarrow M$ é uma curva absolutamente contínua com $\gamma([a, b]) \subset N$ então $\gamma:[a, b] \rightarrow N$ também o é. Segue em particular que se $P \subset M$ é uma outra subvariedade integral de $\mathcal{D}$ com $P \subset N$ então $P$ é um aberto de $N$ (pois a inclusão de $P$ em $N$ é diferenciável e daí segue do teorema da função inversa que a mesma é um difeomorfismo sobre um aberto).

Seja novamente $\mathcal{D}$ uma distribuição integrável em $M$ e considere o conjunto das subvariedades integrais conexas de $\mathcal{D}$ ordenado por inclusão (dizemos que uma subvariedade imersa $N \subset M$ é conexa quando o for na sua própria topologia. Como a inclusão em $M$ é contínua, seguirá também que $N$ é conexa na topologia induzida de $M$ ). Os elementos maximais desse conjunto serão chamados subvariedades integrais maximais de $\mathcal{D}$. $\mathrm{O}$ teorema de Frobenius nos diz também que para todo $m \in M$ existe uma única subvariedade integral maximal $N$ de $\mathcal{D}$ com $m \in N$ (essa, em muitos exemplos, não é mergulhada). Vale que se $f: P \rightarrow N$ é uma função de classe $C^{1}$ (onde $P$ é uma variedade conexa) tal que $d f_{p}\left(T_{p} P\right) \subset \mathcal{D}_{f(p)}$ para todo $p \in P$ e tal que $f(P) \cap N \neq \phi$ então $f(P) \subset N$. Vale também que se $\gamma:[a, b] \rightarrow M$ é uma curva absolutamente contínua com $\dot{\gamma}(t) \in \mathcal{D}_{\gamma(t)}$ para quase todo $t \in[a, b]$ e $\gamma([a, b]) \cap N \neq \phi$ então $\gamma([a, b]) \subset N$. Temos o seguinte lema.

Lema 4.1. Seja $\mathcal{D}$ uma distribuição numa variedade $M$. Se escrevermos $M$ como uma reunião disjunta $M=\bigcup_{\lambda \in L} M_{\lambda}$ de subvariedades integrais conexas de $\mathcal{D}$ então (D é integrável e) $M_{\lambda}$ é uma subvariedade integral maximal de $\mathcal{D}$ para todo $\lambda \in L$. 
Demonstração. Sabemos que $\mathcal{D}$ é integrável pois possui uma subvariedade integral passando por cada ponto de $M$. Seja $\lambda \in L$ e seja $m \in M_{\lambda}$. Sabemos que existe uma subvariedade integral maximal $N$ de $\mathcal{D}$ com $m \in N$. Daí para cada $\mu \in L$ temos $M_{\mu} \subset N$ ou $M_{\mu} \cap N=\phi$, pois $M_{\mu}$ é conexa. Escrevemos então a variedade conexa $N$ como reunião disjunta dos abertos não vazios $M_{\mu} \subset N$ com $\mu$ percorrendo um subconjunto de $L$ que contém $\lambda$. Como $N$ é conexa segue que essa reunião possui um único termo, ou seja, $N=M_{\lambda}$.

A partir de agora retomamos as notações da seção 2 a respeito de quocientes $G / K$ de um grupo de Lie por um subgrupo fechado. Recorde que se temos uma ação $G \times M \rightarrow M$ de um grupo de Lie $G$ numa variedade $M$ então a órbita $G(m)=\{g \cdot m: g \in G\}$ de um ponto $m \in M$ é uma subvariedade imersa de $M$. De fato, se $K$ é o subgrupo (fechado) de isotropia de $m$ então a aplicação $f: G \rightarrow M$ dada por $f(g)=g \cdot m$ passa ao quociente e define uma imersão injetora de $G / K$ em $M$ cuja imagem é $G(m)$. Em particular, para $g \in G$ o espaço tangente a $G(m)$ no ponto $g \cdot m$ é a imagem de $d f_{g}$.

Corolário. Seja $G$ um grupo de Lie e $K \subset G$ um subgrupo fechado. Seja $G^{\prime} \subset G$ um subgrupo de Lie conexo cuja álgebra de Lie $\mathfrak{g}^{\prime}$ é invariante pela representação adjunta $\operatorname{Ad}_{\mid K}$ de $K$ em g. Seja $\mathcal{D}$ a distribuição $G$-invariante em $G / K$ tal que $\mathcal{D}_{1 K}=\bar{\pi}\left(\mathfrak{g}^{\prime}\right)$. Então $\mathcal{D}$ é integrável e para todo $g \in G$ temos que $\pi\left(g G^{\prime}\right)$ é uma subvariedade integral maximal de $\mathcal{D}$.

Demonstração. Em primeiro lugar, temos que $\bar{\pi}\left(\mathfrak{g}^{\prime}\right)$ é invariante pela representação $\overline{\mathrm{Ad}}$ de $K \mathrm{em} \mathfrak{g} / \mathfrak{k}$, donde a distribuição $\mathcal{D}$ realmente é bem definida. Temos que $\pi\left(G^{\prime}\right)$ é uma subvariedade imersa de $G / K$, pois é a órbita do ponto $1 K$ pela ação de $G^{\prime}$ em $G / K$ por translação à esquerda. Pela observação que precede o corolário, vemos que o espaço tangente a $\pi\left(G^{\prime}\right)$ no ponto $1 K$ é a imagem de $d\left(\pi_{\mid G^{\prime}}\right)_{1}$, ou seja, $\bar{\pi}\left(\mathfrak{g}^{\prime}\right)=\mathcal{D}_{1 K}$. Para $g \in G^{\prime}$ temos que o difeomorfismo $\bar{l}_{g}$ de $G / K$ preserva $\mathcal{D}$ e $\pi\left(G^{\prime}\right)$, donde o espaço tangente a $\pi\left(G^{\prime}\right)$ no ponto $g K$ coincide com $\mathcal{D}_{g K}$, i.e., $\pi\left(G^{\prime}\right)$ é uma subvariedade integral de $\mathcal{D}$. Agora, para todo $g \in G$ o difeomorfismo $\bar{l}_{g}$ de $G / K$ preserva $\mathcal{D}$ donde $\bar{l}_{g}\left(\pi\left(G^{\prime}\right)\right)=\pi\left(g G^{\prime}\right)$ é uma subvariedade integral de $\mathcal{D}$ também. Como $G^{\prime}$ é conexo segue que $\pi\left(g G^{\prime}\right)$ também o é.

Para aplicarmos o lema, resta ver que para $g, h \in G$ temos $\pi\left(g G^{\prime}\right) \cap \pi\left(h G^{\prime}\right)=\phi$ ou $\pi\left(g G^{\prime}\right)=\pi\left(h G^{\prime}\right)$. Observe que para $k \in K$ temos $\operatorname{Ad}_{k}\left(\mathfrak{g}^{\prime}\right)=\mathfrak{g}^{\prime}$ e como $G^{\prime}$ é conexo segue $\mathcal{I}_{k}\left(G^{\prime}\right)=k G^{\prime} k^{-1}=G^{\prime}$. Se $\pi\left(g G^{\prime}\right) \cap \pi\left(h G^{\prime}\right) \neq \phi$ então existem $g_{1}^{\prime}, g_{2}^{\prime} \in G^{\prime}, k \in K$ com $g g_{1}^{\prime}=h g_{2}^{\prime} k$, donde:

$$
g=h g_{2}^{\prime} k g_{1}^{\prime-1}=h g^{\prime} k
$$

com $g^{\prime}=g_{2}^{\prime} k g_{1}^{\prime-1} k^{-1} \in G^{\prime}$. Daí:

$$
\pi\left(g G^{\prime}\right)=\pi\left(h g^{\prime} k G^{\prime}\right)=\pi\left(h g^{\prime} G^{\prime} k\right)=\pi\left(h G^{\prime}\right),
$$

pois $k G^{\prime}=G^{\prime} k$.

Observação 4.1. Se $K$ é conexo então a invariância de $\mathfrak{g}^{\prime}$ por $\operatorname{Ad}_{\mid K}$ é equivalente à invariância de $\mathfrak{g}^{\prime}$ por $\operatorname{ad}_{\mathfrak{k}}$, i.e., equivalente a $\left[\mathfrak{k}, \mathfrak{g}^{\prime}\right] \subset \mathfrak{g}^{\prime}$.

Seja $M$ uma variedade sub-Riemanniana. Diremos que $M$ possui a propriedade dos minimizantes regulares (pmr) quando toda curva minimizante pca (parametrizada por comprimento de arco) em $M$ for diferenciável (i.e., de classe $C^{\infty}$ ). 
O corolário do lema 1.4 nos diz que quando $\mathcal{D}$ é fortemente geradora por colchetes (em particular, se $\mathcal{D}$ é de contato) então $M$ possui pmr. O teorema 1.5 nos fornece ainda mais exemplos de variedades sub-Riemannianas que possuem pmr (note que todas as variedades com distribuição homogênea de codimensão 1 possuem pmr, pelo teorema 1.5). O teorema principal da seção (teorema 4.4) nos fornece ainda mais uma família de exemplos. Precisamos de mais alguns lemas preparatórios.

Lema 4.2. Seja $M$ uma variedade sub-Riemanniana e seja $U \subset M$ um aberto. Dado $p \in U$ existe um número $r>0$ tal que toda curva horizontal $\gamma:[a, b] \rightarrow M \operatorname{com} \gamma(a)=p$ e $L(\gamma)<r$ satisfaz $\gamma([a, b]) \subset U$.

Demonstração. Comparamos a métrica sub-Riemanniana $g$ com a métrica euclideana num sistema de coordenadas em torno de $p$. Seja $\varphi: V \rightarrow \tilde{V}$ um sistema de coordenadas em $M$ com $p \in V \subset U$. Seja $B \subset V$ a imagem inversa por $\varphi$ de uma bola fechada $B[\varphi(p) ; s] \subset \tilde{V}$, para algum $s>0$. Para $v \in T_{m} M$ com $m \in V$ denote por $\|v\|_{e}$ a norma euclideana do vetor $d \varphi_{m}(v)$. O conjunto dos vetores $v \in \mathcal{D}$, tangentes em pontos do compacto $B$ e tais que $\|v\|_{e}=1$, formam um subconjunto compacto de $\mathcal{D}$ no qual a função contínua $v \mapsto g(v, v)^{1 / 2}=\|v\|$ assume um mínimo $k>0$. Observe que para todo $v \in \mathcal{D}$, tangente num ponto de $B$, temos $\|v\| \geq k\|v\|_{e}$.

Tome $r=k s>0$. Se $\gamma:[a, b] \rightarrow M$ é uma curva horizontal com $\gamma(a)=p$ e $\gamma([a, b]) \not \subset U$ então existe $c \in(a, b) \operatorname{com} \gamma([a, c]) \subset B$ e $\gamma(c) \in \partial B$ (tome $c$ como sendo o mínimo do conjunto fechado $\left.\gamma^{-1}(\partial B) \neq \phi\right)$. Daí:

$$
L(\gamma) \geq L\left(\gamma_{\mid[a, c]}\right) \geq k L_{e}\left(\varphi \circ \gamma_{\mid[a, c]}\right) \geq k s=r
$$

onde $L_{e}$ denota o comprimento euclideano de uma curva.

Observamos que se $M$ é uma variedade sub-Riemanniana então todo aberto $U \subset M$ possui uma estrutura sub-Riemanniana natural, restringindo $\mathcal{D}$ e $g$.

Corolário 1. Seja $M$ uma variedade sub-Riemanniana. Se $M$ possui pmr então todo aberto de $M$ possui pmr e se $M$ puder ser escrita como reunião de abertos que possuem pmr então $M$ possui pmr (i.e., pmr é uma propriedade local).

Demonstração. Suponha que $M$ possui pmr e seja $U \subset M$ um aberto. Seja $\gamma:[a, b] \rightarrow U$ uma curva minimizante (em $U$ ) pca. Seja $t_{0} \in[a, b]$. Mostremos que $\gamma$ é diferenciável numa vizinhança de $t_{0}$. Seja $p=\gamma\left(t_{0}\right) \in U$ e seja $r>0$ como no lema.

A continuidade da aplicação $t \mapsto \int_{t_{0}}^{t}\|\dot{\gamma}(s)\| d s$ nos mostra que existe um intervalo fechado $I \subset[a, b]$ que contém $t_{0}$ no seu interior (relativo a $[a, b]$ ) tal que $L\left(\gamma_{\mid I}\right)<r / 2$. Daí $\gamma_{\mid I}$ é minimizante (pca) em $M$. De fato, uma curva mais curta que $\gamma_{\mid I}$ (ligando suas extremidades) estaria contida em $U$ e nos permitiria encurtar $\gamma$ em $U$. Como $M$ possui pmr o resultado segue.

Reciprocamente, se escrevermos $M=\bigcup_{\lambda \in L} U_{\lambda}$ onde cada aberto $U_{\lambda} \subset M$ possui pmr então dada uma curva minimizante pca $\gamma:[a, b] \rightarrow M$ escolhemos $t_{0} \in[a, b]$ e restringimos $\gamma$ a um intervalo $I$ contendo $t_{0}$ no seu interior (relativo a $[a, b]$ ) de modo que $\gamma(I) \subset U_{\lambda}$ para algum $\lambda \in L$. É claro que $\gamma_{\mid I}$ é minimizante pca em $U_{\lambda}$ e portanto é diferenciável.

É claro que se $M$ e $N$ são isométricas então $M$ possui pmr se e somente se $N$ possui pmr. Na verdade temos o seguinte. 
Corolário 2. Seja $f: M \rightarrow N$ uma isometria local entre variedades sub-Riemannianas $M$ e $N$. Se $N$ possui pmr então $M$ também possui pmr e se $f$ é sobrejetora vale a recíproca, i.e., se $M$ possui pmr então $N$ também possui $p m r$.

Demonstração. Se $N$ possui pmr então todo aberto de $N$ também possui pmr e $M$ pode ser coberta por abertos isométricos a abertos de $N$. Se $f$ é sobrejetora e $M$ possui pmr então (todo aberto de $M$ possui pmr e) $N$ pode ser coberta por abertos isométricos a abertos de $M$. O resultado segue do corolário 1 .

Lema 4.3. Sejam $(M, \mathcal{D}, g)$ uma variedade sub-Riemanniana e $f: N \rightarrow M$ uma imersão de uma variedade $N$. Para cada $n \in N$, seja $\mathcal{D}_{n}^{\prime}$ a imagem inversa de $\mathcal{D}_{f(n)}$ pela aplicação linear $d f_{n}$. Suponha que a dimensão de $\mathcal{D}_{n}^{\prime}$ não depende de $n \in N$. Defina um produto interno $g_{n}^{\prime}$ em $\mathcal{D}_{n}^{\prime}$ fazendo $g_{n}^{\prime}(v, w)=g_{f(n)}\left(d f_{n}(v), d f_{n}(w)\right)$. Então $\left(N, \mathcal{D}^{\prime}, g^{\prime}\right)$ é uma variedade sub-Riemanniana (i.e., $\mathcal{D}^{\prime}$ e $g^{\prime}$ são diferenciáveis).

Demonstração. Seja $n \in N$ e seja $\theta^{1}, \ldots, \theta^{n-k}$ um referencial de $\mathcal{D}^{0}$ numa vizinhança aberta $U$ de $f(n)$ em $M$. Daí as 1 -formas $f^{*} \theta^{1}, \ldots, f^{*} \theta^{n-k}$ em $f^{-1}(U) \subset N$ geram o anulador de $\mathcal{D}^{\prime}$ em cada ponto. Segue da observação 1.5 que $\mathcal{D}^{\prime}$ é uma distribuição em $N$.

Agora, se $X^{1}, \ldots, X^{c}$ é um referencial de $\mathcal{D}^{\prime}$ então as funções $g^{\prime}\left(X^{i}, X^{j}\right)$ são dadas por $g\left(d f \circ X^{i}, d f \circ X^{j}\right)$ e portanto são diferenciáveis, donde $g^{\prime}$ é diferenciável.

Corolário. Sejam $(M, \mathcal{D}, g)$ uma variedade sub-Riemanniana e $N \subset M$ uma subvariedade imersa. Suponha que a dimensão de $\mathcal{D}_{n}^{\prime}=\mathcal{D}_{n} \cap T_{n} N$ não depende de $n \in N$. Defina um produto interno $g_{n}^{\prime}$ em $\mathcal{D}_{n}^{\prime}$ por restrição de $g_{n}$. Então $\left(N, \mathcal{D}^{\prime}, g^{\prime}\right)$ é uma variedade subRiemanniana. Além do mais, se uma curva minimizante pca $\gamma:[a, b] \rightarrow M$ tem imagem em $N$ e é absolutamente contínua em $N$ então $\gamma$ também é minimizante pca em $N$. Em particular, se $N$ possui pmr então $\gamma$ é diferenciável.

Demonstração. Se tomarmos $f$ como sendo a inclusão de $N$ em $M$, no lema, concluímos que $\left(N, \mathcal{D}^{\prime}, g^{\prime}\right)$ é uma variedade sub-Riemanniana. Observe agora que toda curva horizontal em $N$ é também horizontal em $M$ e que seu comprimento relativo a $N$ e a $M$ é o mesmo. Segue facilmente daí que $\gamma$ é minimizante pca em $N$.

Observação 4.2. Como já foi observado, se $N$ é apenas uma subvariedade imersa de $M$ então $\gamma:[a, b] \rightarrow M$ absolutamente contínua e $\gamma([a, b]) \subset N$ em geral não implica $\gamma:[a, b] \rightarrow N$ absolutamente contínua. Por isso supusemos no corolário que $\gamma$ fosse absolutamente contínua em $N$, explicitamente. Por outro lado, se $N$ for uma subvariedade integral de alguma distribuição integrável então, como já foi observado, basta supor que $\gamma$ seja absolutamente contínua em $M$.

Teorema 4.4. Seja $M$ uma variedade sub-Riemanniana e seja dada uma ação transitiva de um grupo de Lie $G$ em $M$ de modo que a distribuição $\mathcal{D}$ seja $G$-invariante. Suponha que a álgebra de Lie g de $G$ seja semi-simples e admita uma estrutura de $A L I O(\mathfrak{g}, s, B)$. Defina a $e^{\mathfrak{k}}$ como no enunciado do lema 3.3.8. Suponha que tenhamos um ponto $x_{0} \in M$ cujo grupo de isotropia $K$ possui k como álgebra de Lie. Seja o o complemento $\mathcal{K}$-ortogonal de $\mathfrak{k} \oplus \mathfrak{a}$ em $\mathfrak{g}$, onde $\mathcal{K}$ denota a forma de Killing de $\mathfrak{g}$. Suponha que a diferencial da aplicação $g \in G \mapsto g .: x_{0} \in M$ no ponto $1 \in G$ leve o sobre $\mathcal{D}_{x_{0}}$. Então $M$ possui pmr.

Demonstração. A demonstração será feita por indução na dimensão do grupo de Lie $G$. A base é trivial, pode ser feita $\operatorname{com} \operatorname{dim} G=0$. Fixamos então $M$ e $G$ como no enunciado 
do teorema e supomos o teorema válido para variedades onde o grupo de Lie que aparece no enunciado pode ser escolhido com dimensão menor que a de $G$.

Podemos identificar $M$ com $G / K$ através do difeomorfismo $g K \mapsto g \cdot x_{0}$ e obtemos uma única estrutura sub-Riemanniana em $G / K$ que o torna uma isometria. Consideramos então $G / K$ com uma distribuição $G$-invariante $\mathcal{D}$ tal que $\mathcal{D}_{1 K}=\bar{\pi}(\mathfrak{d})$ e com uma métrica arbitrária (que foi induzida de $M$ ). Se $K^{0}$ é a componente conexa de 1 em $K$ então temos um difeomorfismo local sobrejetor $f: G / K^{0} \rightarrow G / K$ dado por $f\left(g K^{0}\right)=g K$. Definimos uma estrutura sub-Riemanniana em $G / K^{0}$ que torna $f$ uma isometria local. Pelo corolário 2 do lema 4.2 , basta mostrar que $G / K^{0}$ possui pmr.

Suporemos então que $K$ é conexo e que temos uma distribuição $G$-invariante $\mathcal{D}$ em $G / K \operatorname{com} \mathcal{D}_{1 K}=\bar{\pi}(\mathfrak{d})$, com uma métrica arbitrária e demonstraremos que $G / K$ possui pmr.

Seja $\gamma:[a, b] \rightarrow G / K$ uma curva minimizante pca. Basta mostrar que $\gamma$ é diferenciável numa vizinhança de cada $t \in[a, b]$. Como toda restrição de $\gamma$ é minimizante pca, podemos supor sem perda de generalidade que existe uma seção local $s: U \subset G / K \rightarrow G$ tal que $\gamma([a, b]) \subset U$. Pelo teorema 1.1 temos que $\gamma$ é um extremal normal ou anormal. Se $\gamma$ é um extremal normal então $\gamma$ é diferenciável. Suponha então que $\gamma$ é um extremal anormal. Seja $\mathfrak{m}$ o complemento $\mathcal{K}$-ortogonal de $\mathfrak{k}$ em $\mathfrak{g}$ (segue dos lemas 3.3.2 e 3.1.1 que $\mathfrak{g}=\mathfrak{k} \oplus \mathfrak{m}$ ). Note que $\mathfrak{d} \subset \mathfrak{m}$. Identificamos a partir de agora $\mathfrak{m} \operatorname{com} \mathfrak{g} / \mathfrak{k}$ através de $\bar{\pi}$ e daí $\mathcal{D}_{1 K}$ fica identificada com $\mathfrak{o}$.

Como no corolário do teorema 2.1 definimos $\mu$ e $\hat{\mu}$. Sabemos então que $\mu(t) \in \mathfrak{d}$ para quase todo $t \in[a, b]$ e que existe uma curva absolutamente contínua que nunca se anula $\lambda:[a, b] \rightarrow \mathfrak{d}^{0} \subset \mathfrak{m}^{*}$ tal que:

$$
\dot{\lambda}(t)=\left(\pi_{\mathrm{m}} \circ \operatorname{ad}_{\hat{\mu}(t)} \circ i_{\mathrm{m}}\right)^{*} \lambda(t),
$$

para quase todo $t \in[a, b]$. Recorde também que (vide observação 2.5):

$$
\mu(t)=s(\gamma(t))^{-1} \dot{\gamma}(t) .
$$

Observe que $\mathfrak{d}$ é simplesmente o complemento $\mathcal{K}$-ortogonal de a em $\mathrm{m}$. Temos que a forma de Killing $\mathcal{K}$ é não degenerada em a (pois segue do lema 3.3.8 que $\mathcal{K}$ é não degenerada em $\mathfrak{h}^{c}$ e portanto não degenerada em $\mathfrak{h}$. Basta observar então que a decomposição $\mathfrak{h}=\mathfrak{t} \oplus \mathfrak{a}$ é $\mathcal{K}$-ortogonal). Concluímos então do lema 3.1.1 que $\mathfrak{m}=\mathfrak{d} \oplus \mathfrak{a}$.

Temos que a forma de Killing $\mathcal{K}$ é não degenerada em $m$ (pois $\mathcal{K}$ é não degenerada em $\mathfrak{g}$ e a decomposição $\mathfrak{g}=\mathfrak{k} \oplus \mathfrak{m}$ é $\mathcal{K}$-ortogonal), donde obtemos um isomorfismo $X \mapsto \mathcal{K}(X, \cdot)$ entre $\mathfrak{m}$ e $\mathfrak{m}^{*}$. Identificamos então $\mathfrak{m}$ com $\mathfrak{m}^{*}$ e $\mathfrak{d}^{0} \subset \mathfrak{m}^{*}$ com $\mathfrak{a} \subset \mathfrak{m}$. Observando que para $X \in \mathfrak{g}$ o operador linear $\pi_{\mathfrak{m}} \circ \operatorname{ad}_{X} \circ i_{\mathfrak{m}}$ em $\mathfrak{m}$ é $\mathcal{K}$-antisimétrico vem:

$$
\dot{\lambda}(t)=-\pi_{\mathrm{m}}([\hat{\mu}(t), \lambda(t)]),
$$

para quase todo $t \in[a, b]$. Como $\mu=\pi_{\mathrm{m}} \circ \hat{\mu}$ segue que $\mu(t)$ e $\hat{\mu}(t)$ diferem por um elemento de $\mathfrak{k}$. Como $\lambda(t) \in \mathfrak{a}$ e $[\mathfrak{k}, \mathfrak{a}]=0$ podemos trocar $\hat{\mu}(t)$ por $\mu(t)$ em (4.4.2). Note também que $[\mathfrak{a}, \mathfrak{d}] \subset \mathfrak{d}$, pois $[\mathfrak{a}, \mathfrak{k}+\mathfrak{a}]=0$ e $\mathfrak{d}$ é o complemento $\mathcal{K}$-ortogonal de $\mathfrak{k}+\mathfrak{a}$. Como $\mathfrak{d} \subset \mathfrak{m}$ podemos desconsiderar a projeção $\pi_{\mathrm{m}}$ em (4.4.2) e obtemos:

$$
\dot{\lambda}(t)=-[\mu(t), \lambda(t)],
$$


para quase todo $t \in[a, b]$.

$\mathrm{O}$ lado esquerdo em (4.4.3) pertence a a e o lado direito pertence a $\mathfrak{d}$. Concluímos então que $\dot{\lambda}=0$ quase sempre, i.e., $\lambda$ é um vetor constante não nulo em a e $\mu(t)$ pertence ao centralizador de $\lambda$ quase sempre.

Seja $\Delta$ o conjunto das raízes da álgebra de Lie complexa semi-simples $\mathfrak{g}^{c}$ em relação à subálgebra de Cartan $\mathfrak{h}^{c}$ (vide lema 3.3.8). Seja $\Delta^{\prime}$ o conjunto das raízes $\alpha \in \Delta$ tais que $\alpha(\lambda)=0$. Note que $\Delta^{\prime} \neq \Delta$ (pois caso contrário $\lambda \neq 0$ estaria no centro de $\mathfrak{g}^{c}$ ). Considere o subespaço complexo de $\mathfrak{g}^{c}$ gerado pelos vetores $H_{\alpha} \in \mathfrak{h}^{c}$ e pelos subespaços $\mathfrak{g}_{\alpha}^{c}$ com $\alpha \in \Delta^{\prime}$. Utilizando (3.3.1) e (3.3.2), vemos que se $\alpha \in \Delta^{\prime}$ então $\bar{\alpha} \in \Delta^{\prime}$ e $\alpha \circ s \in \Delta^{\prime}$, donde esse subespaço é invariante pelo automorfismo $s$ e pela conjugação. Segue que o mesmo é a complexificação de um subespaço real $s$-invariante $\mathfrak{g}^{\prime}$ de $\mathfrak{g}$. Temos:

$$
\mathfrak{g}^{\prime c}=\sum_{\alpha \in \Delta^{\prime}} \mathbb{C} H_{\alpha} \oplus \bigoplus_{\alpha \in \Delta^{\prime}} \mathfrak{g}_{\alpha}^{c}
$$

As relações $\left[\mathfrak{g}_{\alpha}^{c}, \mathfrak{g}_{\beta}^{c}\right] \subset \mathfrak{g}_{\alpha+\beta}^{c}$ e $\left[\mathfrak{g}_{\alpha}^{c}, \mathfrak{g}_{-\alpha}^{c}\right] \subset \mathbb{C} H_{\alpha}$ mostram que $\mathfrak{g}^{\prime c}$ é uma subálgebra (complexa) de $\mathfrak{g}^{c}$ e portanto $\mathfrak{g}^{\prime}$ é uma subálgebra (real) de $\mathfrak{g}$. De (3.2.1) vem:

$$
\mathfrak{z}^{c}(\lambda)=\mathfrak{h}^{c} \oplus \bigoplus_{\alpha \in \Delta^{\prime}} \mathfrak{g}_{\alpha}^{c}
$$

e como $\mu$ toma valores em $\mathfrak{d}$, segue que $\mu$ toma valores no segundo termo da soma direta (4.4.4) (note que $\mathfrak{d}$ é $\mathcal{K}$-ortogonal a $\mathfrak{h}$ e que os dois termos em (4.4.4) são mutuamente $\mathcal{K}$-ortogonais também). Em particular, temos que $\mu$ toma valores em $\mathfrak{g}^{\prime}$. Observe que $\mathfrak{g}^{\prime}$ tem dimensão estritamente menor que a de $\mathfrak{g}$, pois $\Delta^{\prime} \neq \Delta$.

Segue facilmente do lema 3.3.9 e das relações de comutação usuais entre os espaços $\mathfrak{g}_{\alpha}^{c}$ que $\left[\mathfrak{k}^{c}, \mathfrak{g}^{\prime c}\right] \subset \mathfrak{g}^{\prime c}$ e portanto $\left[\mathfrak{k}, \mathfrak{g}^{\prime}\right] \subset \mathfrak{g}^{\prime}$ (observe que se $\alpha \in \Delta$ satisfaz $\alpha_{\mid \mathfrak{a}}=0$ então $\alpha \in \Delta^{\prime}$ ). Seja $G^{\prime}$ o subgrupo de Lie conexo de $G$ associado à álgebra de Lie $\mathfrak{g}^{\prime}$. Como $K$ é conexo, podemos usar o corolário do lema 4.1 (e a observação 4.1) para concluir que a distribuição $G$-invariante $\mathcal{D}^{\prime}$ em $G / K$ tal que $\mathcal{D}_{1 K}^{\prime}=\bar{\pi}\left(\mathfrak{g}^{\prime}\right)$ é involutiva e que suas subvariedades integrais maximais são $\pi\left(g G^{\prime}\right)$ para $g \in G$.

De (4.4.1) segue que $\dot{\gamma}(t)$ pertence a $\mathcal{D}^{\prime}$ quase sempre e portanto $\gamma$ tem imagem inteiramente contida em alguma subvariedade $\pi\left(g G^{\prime}\right)$. Pelo corolário do lema 4.3 (e pela observação 4.2) concluímos que $\gamma$ é minimizante pca em $\pi\left(g G^{\prime}\right)$ (a hipótese de dimensão constante que aparece no corolário segue do fato que $\pi\left(g G^{\prime}\right)$ é subvariedade integral de uma distribuição $G$-invariante e que $\mathcal{D}$ também é $G$-invariante). Basta mostrar então que $\pi\left(g G^{\prime}\right)$ possui pmr. Observe que $\pi\left(g G^{\prime}\right)$ é isométrica a $\pi\left(G^{\prime}\right)$ através de $\bar{l}_{g}$, se for escolhida uma métrica conveniente em $\pi\left(G^{\prime}\right)$.

Considere então $\pi\left(G^{\prime}\right)$ com uma distribuição $G^{\prime}$-invariante cujo valor no ponto $1 K$ é $\bar{\pi}\left(\mathfrak{g}^{\prime}\right) \cap \bar{\pi}(\mathfrak{d})$ (recorde que o espaço tangente a $\pi\left(G^{\prime}\right)$ no ponto $1 K$ é $\bar{\pi}\left(\mathfrak{g}^{\prime}\right)$ ). Considere essa distribuição munida de uma métrica qualquer. A demonstração ficará completa se demonstrarmos que $\pi\left(G^{\prime}\right)$ possui pmr. Utilizaremos a hipótese de indução.

Já observamos que $\mathfrak{g}^{\prime}$ é $s$-invariante. Podemos escrever então $\mathfrak{g}^{\prime}=\mathfrak{g}_{+}^{\prime} \oplus \cdot \mathfrak{g}_{-}^{\prime}$, onde $\mathfrak{g}_{+}^{\prime}=\mathfrak{g}^{\prime} \cap \mathfrak{g}_{+}$e $\mathfrak{g}_{-}^{\prime}=\mathfrak{g}^{\prime} \cap \mathfrak{g}_{-}$. Podemos considerar em $\mathfrak{g}_{-}^{\prime}$ o produto interno dado pela restrição de $B$ que é obviamente invariante pela representação adjunta de $\mathfrak{g}_{+}^{\prime}$. 
Considere a ação de $G^{\prime}$ em $\pi\left(G^{\prime}\right)$ por translação à esquerda. Seja $K^{\prime}=G^{\prime} \cap K$ o subgrupo de isotropia de $1 K$ e seja $N \subset G^{\prime}$ o subgrupo que age trivialmente em $\pi\left(G^{\prime}\right)$, i.e., a interseção dos grupos de isotropia de todos os pontos de $\pi\left(G^{\prime}\right)$. Daí $N$ é normal e fechado em $G^{\prime}$ e $N \subset K^{\prime}$. Podemos então considerar o grupo de Lie $\bar{G}=G^{\prime} / N$ com álgebra de Lie $\overline{\mathfrak{g}}=\mathfrak{g}^{\prime} / \mathfrak{n}$, onde $\mathfrak{n} \subset \mathfrak{g}^{\prime}$ é a álgebra de Lie de $N$ (que é um ideal de $\mathfrak{g}^{\prime}$ ). A ação de $G^{\prime}$ em $\pi\left(G^{\prime}\right)$ passa ao quociente e define uma ação de $\bar{G}$ em $\pi\left(G^{\prime}\right)$ que é efetiva. A isotropia do ponto $1 K$ é $\bar{K}=K^{\prime} / N$. Se $\mathfrak{k}^{\prime}$ e $\overline{\mathfrak{k}}$ denotam as álgebras de Lie de $K^{\prime}$ e $\bar{K}$ respectivamente então $\mathfrak{n} \subset \mathfrak{k}^{\prime}$ e $\overline{\mathfrak{k}}=\mathfrak{k}^{\prime} / \mathfrak{n}$.

É fácil ver que $\mathfrak{k}^{\prime}=\mathfrak{g}^{\prime} \cap \mathfrak{k}$ (observe que dado $X \in \mathfrak{g}^{\prime}$ então $X \in \mathfrak{k}^{\prime}$ se e somente se $\exp (t X) \in K^{\prime}$ para todo $t \in \mathbb{R}$. Mas isso ocorre se e somente se $\exp (t X) \in K$ para todo $t \in \mathbb{R}$, i.e., se e somente se $X \in \mathfrak{k})$. Como $\mathfrak{n} \subset \mathfrak{k}^{\prime}$, segue que $\mathfrak{n} \subset \mathfrak{g}_{+}^{\prime}$, donde $s(\mathfrak{n})=\mathfrak{n}$ e podemos definir um automorfismo involutivo $\bar{s}$ de $\bar{g}$ por passagem ao quociente, i.e., $\bar{s} \circ q=q \circ s$, onde $q: \mathfrak{g}^{\prime} \rightarrow \overline{\mathfrak{g}}$ é a aplicação quociente. Como $\mathfrak{n} \subset \mathfrak{g}_{+}^{\prime}$ segue que $\overline{\mathfrak{g}}=\overline{\mathfrak{g}}_{+} \oplus \overline{\mathfrak{g}}_{-}$, $\operatorname{com} \overline{\mathfrak{g}}_{+}=q\left(\mathfrak{g}_{+}^{\prime}\right)$ e $\overline{\mathfrak{g}}_{-}=q\left(\mathfrak{g}_{-}^{\prime}\right)$. Essa é a decomposição de $\overline{\mathfrak{g}}$ em autoespaços de $\bar{s}$. Podemos identificar $\mathfrak{g}_{-}^{\prime}$ com $\overline{\mathfrak{g}}_{-}$através de $q$ e obtemos então um produto interno $B$ em $\overline{\mathfrak{g}}_{-}$que é visivelmente invariante pela representação adjunta de $\overline{\mathfrak{g}}_{+}$.

Temos então uma ação transitiva do grupo de Lie $\bar{G}$ na variedade sub-Riemanniana $\pi\left(G^{\prime}\right)$, de modo que a distribuição é $\bar{G}$-invariante. A isotropia do ponto $1 K$ é $\bar{K}$. A idéia agora é verificar que $(\overline{\mathfrak{g}}, \bar{s}, B)$ é uma ALIO com $\bar{g}$ semi-simples e verificar que a distribuição de $\pi\left(G^{\prime}\right)$ no ponto $1 K$, i.e., $\bar{\pi}\left(\mathfrak{g}^{\prime}\right) \cap \bar{\pi}(\mathfrak{d})$ satisfaz as hipóteses do teorema. Seguirá então da hipótese de indução que $\pi\left(G^{\prime}\right)$ possui pmr, já que $\operatorname{dim} \bar{G} \leq \operatorname{dim} G^{\prime}<\operatorname{dim} G$. Isso completará a demonstração.

Considere a seguinte decomposição de $\mathfrak{g}^{c}$ :

$$
\mathfrak{g}^{c}=\mathfrak{t}^{c} \oplus \mathfrak{a}^{c} \oplus \bigoplus_{\substack{\alpha \in \Delta \\ \alpha \mid \mathfrak{a}=0}} \mathfrak{g}_{\alpha}^{c} \oplus \bigoplus_{\substack{\alpha \in \Delta \\ \alpha \mid \mathfrak{a} \neq 0}} \mathfrak{g}_{\alpha}^{c}
$$

Observe que os quatro termos da soma direta acima são mutuamente $\mathcal{K}$-ortogonais (recorde que $\mathfrak{g}_{\alpha}^{c}$ é $\mathcal{K}$-ortogonal a $\mathfrak{g}_{\beta}^{c}$ para $\beta \neq-\alpha$ e que $\mathfrak{g}_{+}^{c}$ é $\mathcal{K}$-ortogonal a $\mathfrak{g}_{-}^{c}$ ). Pelo lema 3.3 .9 temos que $\mathfrak{k}^{c}$ corresponde aos primeiro e terceiro termos em (4.4.5). Segue que $\mathfrak{m}^{c}$ corresponde aos segundo e quarto termos e que $\mathfrak{d}^{c}$ corresponde somente ao quarto termo.

Como $\mathfrak{g}^{\prime c}$ é $s$-invariante, temos que $T_{\alpha}, A_{\alpha} \in \mathfrak{g}^{\prime c}$ para $\alpha \in \Delta^{\prime}$. Obtemos então uma decomposição:

$$
\mathfrak{g}^{\prime c}=\sum_{\alpha \in \Delta^{\prime}} \mathbb{C} T_{\alpha} \oplus \sum_{\alpha \in \Delta^{\prime}} \mathbb{C} A_{\alpha} \oplus \bigoplus_{\substack{\alpha \in \Delta \\ \alpha \mid \mathfrak{a}=0}} \mathfrak{g}_{\alpha}^{c} \oplus \bigoplus_{\substack{\alpha \in \Delta^{\prime} \\ \alpha \mid \mathfrak{a} \neq 0}} \mathfrak{g}_{\alpha}^{c}
$$

onde cada termo em (4.4.6) está contido no termo correspondente em (4.4.5). Escreva $\mathfrak{m}^{\prime}=\mathfrak{g}^{\prime} \cap \mathfrak{m}$ e $\mathfrak{d}^{\prime}=\mathfrak{g}^{\prime} \cap \mathfrak{d}$. Segue que $\mathfrak{k}^{\prime c}$ corresponde aos primeiro e terceiro termos em (4.4.6), $\mathrm{m}^{\prime c}$ corresponde aos segundo e quarto termos e $\mathfrak{d}^{\prime c}$ é apenas o quarto termo. Escrevemos também $\mathfrak{t}^{\prime}=\mathfrak{g}^{\prime} \cap \mathbf{t}$ e $\mathfrak{a}^{\prime}=\mathfrak{g}^{\prime} \cap \mathfrak{a}$ e é claro que $\mathfrak{t}^{\prime c}$ e $\mathfrak{a}^{\prime c}$ correspondem ao primeiro e ao segundo termo em (4.4.6), respectivamente. Podemos também definir $\mathfrak{h}^{\prime}=\mathfrak{g}^{\prime} \cap \mathfrak{h}$. Daí $\mathfrak{h}^{\prime}=\mathfrak{t}^{\prime} \oplus \mathfrak{a}^{\prime}$ e $\mathfrak{h}^{\prime c}=\sum_{\alpha \in \Delta^{\prime}} \mathbb{C} H_{\alpha}$.

Analisando as decomposições (4.4.5) e (4.4.6) é fácil ver que $\left(\mathfrak{g}^{\prime}+\mathfrak{k}\right) \cap(\mathfrak{d}+\mathfrak{k})=\mathfrak{d}^{\prime}+\mathfrak{k}$ (verifique a igualdade para as complexificações dos espaços correspondentes). Segue daí que $\bar{\pi}\left(\mathfrak{g}^{\prime}\right) \cap \bar{\pi}(\mathfrak{d})=\bar{\pi}\left(\mathfrak{d}^{\prime}\right)$, i.e., a distribuição de $\pi\left(G^{\prime}\right)$ no ponto $1 K$ é simplesmente $\bar{\pi}\left(\mathfrak{d}^{\prime}\right)$. 
Vamos verificar agora que $\mathfrak{a}^{\prime}$ é abeliana maximal em $\mathfrak{g}_{-}^{\prime}$ e que o centralizador de $\mathfrak{a}^{\prime}$ em $\mathfrak{g}_{+}^{\prime}$ é $\mathfrak{k}^{\prime}$. Seja $\alpha \in \Delta^{\prime}$. Temos que $\alpha$ se anula em $\mathfrak{a}$ se e somente se $\alpha$ se anula em $\mathfrak{a}^{\prime}$. De fato, se $\alpha$ se anula em a então obviamente $\alpha$ se anula em $\mathfrak{a}^{\prime}$ e se $\alpha$ se anula em $\mathfrak{a}^{\prime}$ então em particular $\alpha\left(A_{\alpha}\right)=0$ (pois $\left.\alpha \in \Delta^{\prime}\right)$. Daí $\mathcal{K}\left(H_{\alpha}, A_{\alpha}\right)=\mathcal{K}\left(A_{\alpha}, A_{\alpha}\right)=0$ e logo $A_{\alpha}=0$ (vide observação 3.3.3). Daí $H_{\alpha} \in \mathrm{t}^{c}$ e $\operatorname{logo} \alpha=\mathcal{K}\left(H_{\alpha}, \cdot\right)$ se anula em a.

É fácil ver que o centralizador de $\mathfrak{a}^{\prime}$ em $\mathfrak{g}^{\prime c}$ é dado por:

$$
\mathfrak{z}_{\mathfrak{g}^{\prime c}}\left(\mathfrak{a}^{\prime}\right)=\mathfrak{t}^{\prime c} \oplus \mathfrak{a}^{\prime c} \oplus \bigoplus_{\substack{\alpha \in \Delta^{\prime} \\ \alpha \mid \mathfrak{a}^{\prime}=0}} \mathfrak{g}_{\alpha}^{c}=\mathfrak{t}^{\prime c} \oplus \mathfrak{a}^{\prime c} \oplus \bigoplus_{\substack{\alpha \in \Delta \\ \alpha \mid \mathfrak{a}=0}} \mathfrak{g}_{\alpha}^{c},
$$

onde o primeiro e o terceiro termos estão contidos em $\mathfrak{g}_{+}^{\prime c}$ (formando $\mathfrak{k}^{\prime c}$ ) e o segundo está contido em $\mathfrak{g}_{-}^{\prime}{ }^{c}$. Segue o resultado desejado.

Seja $\mathfrak{n}^{\perp}$ o complemento $\mathcal{K}$-ortogonal de $\mathfrak{n}$ em $\mathfrak{g}^{\prime}$. É fácil ver que $\mathfrak{n}^{\perp}$ também é um ideal de $\mathfrak{g}^{\prime}$. Além do mais, como $\mathcal{K}$ é definida negativa em $\mathfrak{n}$ (observe que $\mathfrak{n} \subset \mathfrak{g}_{+}$e use o lema 3.3.2), segue do lema 3.1.1 que $\mathfrak{g}^{\prime}=\mathfrak{n} \oplus \mathfrak{n}^{\perp}$ (soma direta de ideais). Defina $\overline{\mathfrak{a}}=q\left(\mathfrak{a}^{\prime}\right)$ e $\overline{\mathfrak{d}}=q\left(\mathfrak{d}^{\prime}\right)$ (recorde que já definimos $\overline{\mathfrak{E}}$ como sendo a álgebra de Lie de $\bar{K}$, que coincide $\left.\operatorname{com} q\left(\mathfrak{k}^{\prime}\right)\right)$.

Para $X, Y \in \mathfrak{g}_{-}^{\prime}$ temos sempre $[X, Y] \in \mathfrak{n}^{\perp}$ (pois $\mathfrak{g}_{-}^{\prime}$ está contido em $\mathfrak{n}^{\perp}$ ). Em particular $[q(X), q(Y)]=0$ em $\overline{\mathfrak{g}}$ se e somente se $[X, Y]=0$. Segue que $\overline{\mathfrak{a}}$ é abeliana maximal em $\overline{\mathfrak{g}}_{-}$. Do mesmo modo, para $X \in \mathfrak{g}_{+}^{\prime}$ e $Y \in \mathfrak{g}_{-}^{\prime}$ temos sempre $[X, Y] \in \mathfrak{g}_{-}^{\prime}$ (com $\left.\mathfrak{g}_{-}^{\prime} \cap \mathfrak{n}=0\right)$, donde $[q(X), q(Y)]=0$ se e somente se $[X, Y]=0$. Segue que o centralizador de $\overline{\mathfrak{a}}$ em $\overline{\mathfrak{g}}_{+}$é $\overline{\mathfrak{k}}$. As decomposições (4.4.5) e (4.4.6) mostram que $\mathfrak{g}^{\prime}=\mathfrak{k}^{\prime} \oplus \mathfrak{m}^{\prime}$ e $\mathfrak{m}^{\prime}=\mathfrak{d}^{\prime} \oplus \mathfrak{a}^{\prime}$. Segue facilmente que $\overline{\mathfrak{g}}=\overline{\mathfrak{k}} \oplus \overline{\mathfrak{m}}$ e $\overline{\mathfrak{m}}=\overline{\mathfrak{d}} \oplus \overline{\mathfrak{a}}$ (use que $\mathfrak{n} \subset \mathfrak{k}^{\prime}$ ).

Para terminar a demonstração de que $(\overline{\mathfrak{g}}, \bar{s}, B)$ é uma $\mathrm{ALIO}$, resta ver que $\overline{\mathfrak{g}}_{+}$não contém ideais não nulos de $\overline{\mathfrak{g}}$. Seja $\mathfrak{i} \subset \overline{\mathfrak{g}}_{+}$um ideal de $\overline{\mathfrak{g}}$. Por um lado temos $\left[\mathfrak{i}, \overline{\mathfrak{g}}_{-}\right] \subset \mathfrak{i} \subset \overline{\mathfrak{g}}_{+}$, pois i é um ideal. Por outro lado $\left[\mathfrak{i}, \overline{\mathfrak{g}}_{-}\right] \subset \overline{\mathfrak{g}}_{-}$, donde $\left[\mathfrak{i}, \overline{\mathfrak{g}}_{-}\right]=0$. Daí para $X \in q^{-1}(\mathfrak{i}) \subset \mathfrak{g}_{+}^{\prime}$ e para $Y \in \mathfrak{g}_{-}^{\prime}$ temos $[X, Y] \in \mathfrak{n}$ e $[X, Y] \in \mathfrak{g}_{-}^{\prime}$ donde $[X, Y]=0$. Daí $q^{-1}(\mathfrak{i}) \subset \mathfrak{g}_{+}^{\prime}$ centraliza $\mathfrak{g}_{-}^{\prime}$ e em particular centraliza $\mathfrak{a}^{\prime}$, donde $q^{-1}(\mathfrak{i}) \subset \mathfrak{k}^{\prime}$ e $\mathfrak{i} \subset \overline{\mathfrak{k}}$. Segue do lema 3.3 .3 que $\mathfrak{i}=0$ se considerarmos a ação transitiva e efetiva de $\bar{G}$ em $\pi\left(G^{\prime}\right)$ (notando que a álgebra de Lie da isotropia $\bar{K}$ do ponto $1 K$ é $\overline{\mathfrak{k}})$.

O próximo passo é provar que $\overline{\mathfrak{g}}$ é semi-simples. Pelo lema 3.3.5, basta mostrar que se $X \in \overline{\mathfrak{g}}_{-}$centraliza $\overline{\mathfrak{g}}_{-}$então $X=0$. Como já observamos, $\left[\mathfrak{g}_{-}^{\prime}, \mathfrak{g}_{-}^{\prime}\right] \subset \mathfrak{n}^{\perp}$, donde basta mostrar que se $X \in \mathfrak{g}_{-}^{\prime}$ centraliza $\mathfrak{g}_{-}^{\prime}$ então $X=0$.

Seja então $X \in \mathfrak{g}_{-}^{\prime}$ e suponha que $X$ centraliza $\mathfrak{g}_{-}^{\prime}$. Seja $\mathcal{L}$ a subálgebra de Lie complexa gerada por $\mathfrak{g}_{-}^{\prime}$ em $\mathfrak{g}^{c}$. Como $\mathfrak{z}_{\mathfrak{g}^{c}}(X)$ é uma subálgebra complexa, segue que $X$ centraliza $\mathcal{L}$. Seja $\alpha \in \Delta^{\prime} \operatorname{com} \alpha_{\mid \mathfrak{a}} \neq 0$. Mostremos que $\mathfrak{g}_{\alpha}^{c} \subset \mathcal{L}$. Já mostramos acima que se $\alpha \in \Delta^{\prime}$ e $\alpha_{\mid \mathfrak{a}} \neq 0$ então na verdade $\alpha_{\mid \mathfrak{a}^{\prime}} \neq 0$. Podemos então escolher $H \in \mathfrak{a}^{\prime c} \subset \mathcal{L}$ com $\alpha(H)=1$. Seja $Y \in \mathfrak{g}_{\alpha}^{c}$. Mostremos que $Y \in \mathcal{L}$. Como $\alpha \in \Delta^{\prime}$ temos $Y \in \mathfrak{g}^{\prime c}$ e portanto $Y-s(Y) \in \mathfrak{g}_{-}^{\prime c} \subset \mathcal{L}$. Mas:

$$
[H, Y-s(Y)]=Y+s(Y) \in \mathcal{L},
$$

lembrando que $s(Y) \in \mathfrak{g}_{\alpha \circ s}^{c}$ e $s(H)=-H$. De $Y-s(Y) \in \mathcal{L}$ e $Y+s(Y) \in \mathcal{L}$ vem $Y \in \mathcal{L}$.

Como $X \in \mathfrak{g}_{-}^{\prime}$ centraliza $\mathfrak{a}^{\prime}$ temos $X \in \mathfrak{a}^{\prime}$. Daí para toda raiz $\alpha \in \Delta^{\prime}$ temos $\alpha(X)=0$ (se $\alpha_{\mid \mathfrak{a}} \neq 0$ então basta notar que $X$ centraliza $\mathfrak{g}_{\alpha}^{c}$ e se $\alpha_{\mid \mathfrak{a}}=0$ é claro que $\alpha(X)=0$ ). Mas 
daí $\mathcal{K}\left(H_{\alpha}, X\right)=0$ sempre que $\alpha \in \Delta^{\prime}$ e segue que $X \in \mathfrak{h}^{\prime}$ é $\mathcal{K}$-ortogonal a $\mathfrak{h}^{\prime}$. Como $\mathcal{K}$ é não degenerada em $\mathfrak{h}^{\prime}$ segue que $X=0$ (observe que $\sum_{\alpha \in \Delta^{\prime}} \mathbb{R} H_{\alpha}$ é uma forma real de $\mathfrak{h}^{\prime c}$ onde $\mathcal{K}$ é definida positiva).

Sejam agora $\mathcal{K}^{\prime}$ e $\overline{\mathcal{K}}$ as formas de Killing de $\mathfrak{g}^{\prime}$ e $\overline{\mathfrak{g}}$, respectivamente (e também de $\mathfrak{g}^{\prime c} \mathrm{e}$ $\overline{\mathfrak{g}}^{c}$, respectivamente). Note que $\mathcal{K}^{\prime}$ em geral não é a restrição de $\mathcal{K}$. Resta apenas mostrar que $\overline{\mathfrak{d}}$ é o complemento $\overline{\mathcal{K}}$-ortogonal de $\overline{\mathfrak{k}} \oplus \overline{\mathfrak{a}}$ em $\overline{\mathfrak{g}}$. Começamos estudando as relações de $\mathcal{K}^{\prime}$-ortogonalidade em $\mathfrak{g}^{\prime}$.

Sejam $\alpha, \beta \in \Delta^{\prime}$ tais que $\alpha+\beta$ não se anula identicamente em $\mathfrak{h}^{\prime}$. Daí $\mathfrak{g}_{\alpha}^{c}$ e $\mathfrak{g}_{\beta}^{c}$ são $\mathcal{K}^{\prime}$-ortogonais. De fato, fixe $H \in \mathfrak{h}^{\prime} \operatorname{com}(\alpha+\beta)(H) \neq 0$ e escolha $X \in \mathfrak{g}_{\alpha}^{c}, Y \in \mathfrak{g}_{\beta}^{c}$. Temos:

$$
\mathcal{K}^{\prime}([H, X], Y)+\mathcal{K}^{\prime}(X,[H, Y])=0 \Rightarrow(\alpha+\beta)(H) \mathcal{K}^{\prime}(X, Y)=0
$$

O raciocínio acima mostra também que $\mathfrak{h}^{\prime c}$ é $\mathcal{K}^{\prime}$-ortogonal a $\mathfrak{g}_{\alpha}^{c}$ para $\alpha \in \Delta^{\prime}$ (escolha $X \in \mathfrak{h}^{\prime c}, Y \in \mathfrak{g}_{\alpha}^{c}$ e fixe $H=H_{\alpha} \in \mathfrak{h}^{\prime c}$. Note que então $\left.\alpha(H)>0\right)$.

Temos que $s$ se restringe a um automorfismo de $\mathfrak{g}^{\prime}$, donde $\mathcal{K}^{\prime}(s(X), s(Y))=\mathcal{K}^{\prime}(X, Y)$ para $X, Y \in \mathfrak{g}^{\prime}$. Fazendo $X \in \mathfrak{g}_{+}^{\prime}$ e $Y \in \mathfrak{g}_{-}^{\prime}$ vemos que $\mathfrak{g}_{+}^{\prime}$ é $\mathcal{K}^{\prime}$-ortogonal a $\mathfrak{g}_{-}^{\prime}$. As considerações feitas até agora sobre $\mathcal{K}^{\prime}$ mostram que os quatro termos na decomposição (4.4.6) são dois a dois $\mathcal{K}^{\prime}$-ortogonais (recorde novamente que uma raiz em $\Delta^{\prime}$ que não é nula em a também não é nula em $\left.\mathfrak{a}^{\prime}\right)$.

Concluímos que as decomposições $\mathfrak{g}^{\prime}=\mathfrak{k}^{\prime} \oplus \mathfrak{m}^{\prime}$ e $\mathfrak{m}^{\prime}=\mathfrak{d}^{\prime} \oplus \mathfrak{a}^{\prime}$ são $\mathcal{K}^{\prime}$-ortogonais. Observe agora que a aplicação quociente $q$ se restringe a um isomorfismo entre as álgebras de Lie $\mathfrak{n}^{\perp}$ e $\overline{\mathfrak{g}}$. Como $\mathfrak{n}^{\perp}$ é um ideal de $\mathfrak{g}^{\prime}$, segue que sua forma de Killing é simplesmente a restrição de $\mathcal{K}^{\prime}$. Daí $\overline{\mathcal{K}}(q(X), q(Y))=\mathcal{K}^{\prime}(X, Y)$, desde que os representantes $X$ e $Y$ das classes $q(X)$ e $q(Y)$ sejam escolhidos em $\mathfrak{n}^{\perp}$. Não é difícil ver agora que as decomposições $\overline{\mathfrak{g}}=\overline{\mathfrak{k}} \oplus \overline{\mathfrak{m}}$ e $\overline{\mathfrak{m}}=\overline{\mathfrak{d}} \oplus \overline{\mathfrak{a}}$ são $\overline{\mathcal{K}}$-ortogonais (note que $\mathfrak{n} \subset \mathfrak{k}^{\prime}$ e $\mathfrak{m}^{\prime} \subset \mathfrak{n}^{\perp}$ ).

Temos agora uma decomposição $\overline{\mathcal{K}}$-ortogonal $\overline{\mathfrak{g}}=\overline{\mathfrak{k}} \oplus \overline{\mathfrak{a}} \oplus \overline{\mathfrak{d}}$. Como $\overline{\mathcal{K}}$ é não degenerada em $\overline{\mathfrak{k}} \oplus \overline{\mathfrak{a}}$ (isso é um fato geral para ALIOs semi-simples), segue que $\overline{\mathfrak{d}}$ é o complemento $\overline{\mathcal{K}}$-ortogonal de $\overline{\mathfrak{k}} \oplus \overline{\mathfrak{a}}$.

O teorema 4.4 nos fornece uma família de exemplos de variedades que possuem pmr. Para construir um tal exemplo procedemos da seguinte forma. Começamos com uma ALIO $(\mathfrak{g}, s, B)$ com $\mathfrak{g}$ semi-simples. Tais ALIOs estão completamente classificadas em [W]. Definimos a e $\mathfrak{k}$ como no enunciado do lema 3.3 .8 e consideramos $\mathfrak{d}$ como sendo $o$ complemento $\mathcal{K}$-ortogonal de $\mathfrak{k}+\mathfrak{a}$ em $\mathfrak{g}$ (temos então $\mathfrak{g}=\mathfrak{k} \oplus \mathfrak{d} \oplus \mathfrak{a}$, como foi visto na demonstração do teorema 4.4).

Considere um grupo de Lie $G$ qualquer cuja álgebra de Lie é g. Seja $K \subset G$ o subgrupo conexo associado à subálgebra $\mathfrak{k} \subset \mathfrak{g}$. Mostraremos no lema 4.5 que $K$ é fechado em $G$ e portanto podemos considerar a variedade $M=G / K$. Seja $\mathcal{D}$ a única distribuição $G$-invariante em $M$ tal que $\mathcal{D}_{1 K}=\bar{\pi}(\mathfrak{d}) \subset \mathfrak{g} / \mathfrak{k}$. Tal distribuição sempre existe, já que é fácil ver que $[\mathfrak{k}, \mathfrak{o}] \subset \mathfrak{o}$.

$\mathrm{Na}$ verdade, podemos também considerar um subgrupo de Lie desconexo $K \subset G$ com álgebra de Lie $\mathfrak{k}$, mas daí a existência de $\mathcal{D}$, i.e., o fato que $\bar{\pi}(\mathcal{O})$ é invariante pela representação isotrópica $\overline{\mathrm{Ad}}$ de $K$ em $\mathfrak{g} / \mathfrak{k}$ não segue simplesmente de $[\mathfrak{k}, \mathfrak{o}] \subset \mathfrak{d}$ e deve ser verificado diretamente em cada exemplo. Quando $K$ tem apenas um número finito de componentes conexas, seguirá automaticamente que $K$ é fechado em $G$, mas quando tivermos 
infinitas componentes conexas em $K$, também será necessário verificar diretamente se $K$ é fechado em $G$.

Finalmente, consideramos qualquer métrica sub-Riemanniana $g$ em $(M, \mathcal{D})$ e o teorema 4.4 nos diz que essa variedade possui pmr. É claro que (a menos de isometrias) construímos assim todos os exemplos nos quais o teorema 4.4 se aplica. Observe que o produto interno $B$ que aparece na $\operatorname{ALIO}(\mathfrak{g}, s, B)$ não tem nenhuma relevância, apenas sua existência é importante para que possamos usar a teoria de ALIOs. Mostramos agora que o subgrupo $K$ é de fato fechado em $G$.

Observação 4.3. Seja $G$ um grupo de Lie e $H \subset G$ um subgrupo de Lie. Sejam $\mathfrak{g}$, h suas álgebras de Lie, respectivamente. Dado $X \in \mathfrak{g}$, temos $X \in \mathfrak{h}$ se e somente se $\exp (t X) \in H$ para todo $t \in \mathbb{R}$. Essa técnica de calcular álgebras de Lie nos fornece os dois seguintes resultados que serão usados no lema seguinte. Se $f: G \rightarrow H$ é um homomorfismo de grupos de Lie e se $K \subset H$ é um subgrupo fechado então $f^{-1}(K) \subset G$ é um subgrupo fechado cuja álgebra de Lie é $d f_{1}^{-1}(\mathfrak{k})$, onde k é a álgebra de Lie de $K$. Também, se $f: G \rightarrow G$ é um homomorfismo então seu conjunto de pontos fixos é um subgrupo fechado de $G$ cuja álgebra de Lie é o conjunto de pontos fixos de $d f_{1}$.

Lema 4.5. Seja $G$ um grupo de Lie cuja álgebra de Lie possui uma estrutura de ALIO $(\mathfrak{g}, s, B)$. Suponha que $\mathfrak{g}$ é semi-simples e defina $\mathfrak{k}$ e a como no enunciado do lema 3.3.8. Seja $K \subset G$ o subgrupo de Lie conexo associado à subálgebra $\mathfrak{k} \subset \mathfrak{g}$. Então $K$ é fechado em $G$.

Demonstração. Seja $G_{+} \subset G$ o subgrupo de Lie conexo associado à subálgebra $\mathfrak{g}_{+}$. Considere o homomorfismo de $G_{+}$em GL(g) dado pela restrição da representação adjunta Ad de $G$ em g. O conjunto dos elementos de $G L(g)$ que fixam os pontos de a é um subgrupo fechado de $\mathrm{GL}(\mathfrak{g})$ cuja álgebra de Lie é formada pelos elementos de $\mathcal{L}(\mathfrak{g})$ que se anulam em a. Segue da observação 4.3 que o conjunto:

$$
K^{\prime}=\left\{g \in G_{+}:\left(\operatorname{Ad}_{g}\right)_{\mid \mathbf{a}}=\mathrm{Id}\right\}
$$

é um subgrupo fechado de $G_{+}$cuja álgebra de Lie é o centralizador de a em $\mathfrak{g}_{+}$, ou seja, k. Daí $K$ é a componente conexa de 1 de $K^{\prime}$ e portanto também é fechado em $G_{+}$.

Resta mostrar que $G_{+}$é fechado em $G$. Como $G_{+}$é conexo, segue que o mesmo está contido na componente conexa de 1 de $G$ e portanto podemos supor sem perda de generalidade que $G$ é conexo. Seja $q: \tilde{G} \rightarrow G$ o recobrimento universal de $G$. Seja $N=$ Ker $q$. Daí $N$ é um subgrupo normal e discreto de $\tilde{G}$ e portanto está contido no centro $Z(\tilde{G})$ de $\tilde{G}$. Como $\mathfrak{g}$ é semi-simples segue que $\mathfrak{z}(\mathfrak{g})=0$ e portanto $Z(\tilde{G})$ é um subgrupo (normal, fechado e) discreto de $\tilde{G}$. Considere o grupo de Lie $\hat{G}=\tilde{G} / Z(\tilde{G})$. Seja $q^{\prime}: G \rightarrow \hat{G}$ a aplicação quociente.

Como $N$ está contido em $Z(\tilde{G})$, vemos que $q^{\prime}$ passa ao quociente e define um homomorfismo $f: G \rightarrow \hat{G}$ tal que $f \circ q=q^{\prime}$. Note que $\tilde{G}, G$ e $\hat{G}$ todos possuem álgebra de Lie $g$ e que $d f_{1}=$ Id.

Como $\tilde{G}$ é simplesmente conexo, o automorfismo $s$ de g se integra a um automorfismo $S$ de $\tilde{G}$ tal que $d S_{1}=s$. É claro que $S(Z(\tilde{G}))=Z(\tilde{G})$ e portanto $S$ passa ao quociente e define um automorfismo $\hat{S}$ de $\hat{G}$ tal que $\hat{S} \circ q^{\prime}=q^{\prime} \circ S$. Daí $d \hat{S}_{1}=s$. Seja $\hat{G}_{+}$a componente conexa de 1 do conjunto de pontos fixos de $\hat{S}$. Pela observação $4.3, \hat{G}_{+}$é um subgrupo 
fechado de $\hat{G}$ com álgebra de Lie $\mathfrak{g}_{+}$. Novamente pela observação 4.3 , segue que $G_{+}$é a componente conexa de 1 de $f^{-1}\left(\hat{G}_{+}\right)$e portanto é fechado em $G$.

Exemplo 4.1. Seja $\mathfrak{g}=\operatorname{sl}(n, \mathbb{R})$ a álgebra de Lie de matrizes reais $n \times n$ de traço zero. Seja $s \in \operatorname{Aut}(\mathfrak{g})$ dado por $s(A)=-A^{t}$. Então $s$ é um automorfismo involutivo

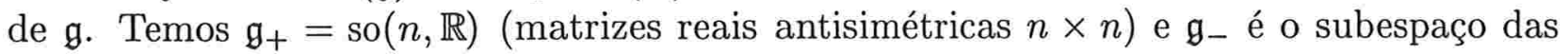
matrizes reais simétricas $n \times n$ de traço zero. Defina o produto interno $B$ em $g_{-}$fazendo $B(X, Y)=\operatorname{tr}(X Y)$. Daí $(\mathfrak{g}, s, B)$ é uma ALIO (vide observação 3.3.1 para mostrar que $\mathfrak{g}_{+}$ não contém ideais não nulos de $\mathfrak{g})$ e $\mathfrak{g}$ é semi-simples.

Seja $\mathfrak{a} \subset \mathfrak{g}_{-}$a subálgebra abeliana formada pelas matrizes diagonais (de traço zero). Daí a é abeliana maximal em $\mathfrak{g}_{-}$. O centralizador de $\mathfrak{a}$ em $\mathfrak{g}_{+}$é $\mathfrak{k}=0$. A forma de Killing de $\mathfrak{g}$ é dada por $\mathcal{K}(X, Y)=2 n \operatorname{tr}(X Y)$. Segue que $\mathfrak{d} \subset \mathfrak{g}$ é o subespaço formado pelas matrizes com zeros na diagonal principal.

Seja $G=\operatorname{SL}(n, \mathbb{R})$ o grupo de Lie de matrizes reais $n \times n$ de determinante 1 . Daí $G$ é um grupo de Lie com álgebra de Lie g. Tomamos $K=\{\mathrm{Id}\}$ e daí $M=G$. Temos que $\mathcal{D}$ é simplesmente a distribuição invariante à esquerda tal que $\mathcal{D}_{1}=\mathfrak{o}$. O teorema 4.4 nos diz que $(M, \mathcal{D})$ possui pmr (para qualquer métrica sub-Riemanniana). Observamos que um resultado desse tipo foi mostrado em [Mg3] para grupos de Lie compactos semi-simples, mas aqui $G$ não é compacto.

Seguindo as construções do lema 3.3.8, temos que $t=0$ é uma subálgebra abeliana maximal de $\mathfrak{k}=0$ e $\mathfrak{h}=\mathfrak{t}+\mathfrak{a}=\mathfrak{a}$. Agora $\mathfrak{g}^{c}=\operatorname{sl}(n, \mathbb{C})$ (matrizes complexas $n \times n$ de traço zero) é uma álgebra de Lie complexa semi-simples e $\mathfrak{h}^{c}$ é a subálgebra de matrizes diagonais complexas (de traço zero). Daí $\mathfrak{h}^{c}$ é uma subálgebra de Cartan de $\mathfrak{g}^{c}$.

Para $i, j=1, \ldots, n$, seja $E_{i j}$ a matriz com entrada 1 na linha $i$, coluna $j$, e zeros nas outras entradas. As matrizes $E_{i j}$ para $i \neq j$ e $E_{i i}-E_{n n}$ para $i<n$ formam uma base de $\mathfrak{g}$ $\left(\right.$ e de $\left.\mathfrak{g}^{c}\right)$. As matrizes $E_{i i}-E_{n n}, i<n$, formam uma base de $\mathfrak{h}\left(\mathrm{e}\right.$ de $\left.\mathfrak{h}^{c}\right)$. Seja $\theta_{i} \in \mathfrak{h}^{c *}$ o funcional dado por $\theta_{i}(H)=H_{i i}$. Daí $\theta_{1}, \ldots, \theta_{n-1}$ formam uma base de $\mathfrak{h}^{c *}$ e $\sum_{i=1}^{n} \theta_{i}=0$.

Se $H \in \mathfrak{h}^{c}$ então $\left[H, E_{i j}\right]=\left(H_{i i}-H_{j j}\right) E_{i j}$, para $i \neq j$. Isso mostra que $\alpha=\theta_{i}-\theta_{j}$ é uma raiz de $\mathfrak{g}^{c}$ e que $\mathfrak{g}_{\alpha}^{c}$ é gerado por $E_{i j}$. Temos:

$$
\Delta=\left\{\theta_{i}-\theta_{j}: i, j=1, \ldots, n, i \neq j\right\} .
$$

Também:

$$
\mathfrak{g}^{c}=\mathfrak{h}^{c} \oplus \bigoplus_{\alpha \in \Delta} \mathfrak{g}_{\alpha}^{c}
$$

o que mostra realmente que $\Delta$ é o conjunto das raízes de $\mathfrak{g}^{c}$ em relação a $\mathfrak{h}^{c}$.

Observe que $\mathfrak{h}$ é uma forma real de $\mathfrak{h}^{c}$ onde as raízes assumem valores reais e portanto $\mathfrak{h}=\mathfrak{h}_{\mathbb{R}}$. Observe também que para $\alpha=\theta_{i}-\theta_{j}$ temos $H_{\alpha}=\frac{1}{2 n}\left(E_{i i}-E_{j j}\right)$.

Podemos determinar concretamente neste exemplo a subálgebra $\mathfrak{g}^{\prime} \subset \mathfrak{g}$ e o subgrupo $G^{\prime} \subset G$ que aparecem na demonstração do teorema 4.4. Como nesse exemplo $K$ é trivial, teremos também que $N$ é trivial e $\bar{G}=G^{\prime}$, i.e., $\left(\mathfrak{g}^{\prime}, s, B\right)$ é uma ALIO (não há necessidade de fazer um quociente para eliminar os ideais de $\mathfrak{g}^{\prime}$ contidos em $\left.\mathfrak{g}_{+}^{\prime}\right)$.

Seja $\lambda \in \mathfrak{a}, \lambda \neq 0$. Para $c_{1}, \ldots, c_{n} \in \mathbb{C}$ denotamos por $\operatorname{Diag}\left(c_{1}, \ldots, c_{n}\right)$ a matriz $\sum_{i=1}^{n} c_{i} E_{i i}$. Mudando de base, se necessário, podemos supor que:

$$
\lambda=\operatorname{Diag}(\underbrace{\lambda_{1}, \ldots, \lambda_{1}}_{n_{1} \text { vezes }}, \ldots, \underbrace{\lambda_{r}, \ldots, \lambda_{r}}_{n_{r} \text { vezes }}),
$$


onde $\lambda_{1}, \ldots, \lambda_{r} \in \mathbb{C}$ são distintos, $\sum_{i=1}^{r} n_{i}=n$ e $\sum_{i=1}^{r} n_{i} \lambda_{i}=0$.

Não é difícil ver que $\mathfrak{g}^{\prime}=\operatorname{sl}\left(n_{1}, \mathbb{R}\right) \oplus \cdots \oplus \operatorname{sl}\left(n_{r}, \mathbb{R}\right)$ (i.e., $r$ matrizes de traço zero emendadas ao longo da diagonal principal) e que $G^{\prime}=\operatorname{SL}\left(n_{1}, \mathbb{R}\right) \times \cdots \times \operatorname{SL}\left(n_{r}, \mathbb{R}\right)$ (i.e., $r$ matrizes de determinante 1 emendadas ao longo da diagonal principal). Temos que todo extremal anormal de $M=G$ tem imagem contida numa classe à esquerda de algum subgrupo desse tipo (i.e., para valores apropriados de $n_{1}, \ldots, n_{r}$ ).

Durante o processo indutivo, refinamos cada vez mais a divisão de $\operatorname{SL}(n, \mathbb{R})$ em blocos emendados pela diagonal principal, até que a curva minimizante pca com a qual começamos torne-se um extremal normal da subvariedade em questão. O processo para também quando os blocos em questão tem lado 1, o que implica que a curva considerada é constante. Isso ilustra a demonstração do teorema 4.4.

Em geral, variedades sub-Riemannianas onde $\mathcal{D}$ não é geradora por colchetes são consideradas "pouco interessantes" (vide observação 1.1). Queremos mostrar agora que todos os exemplos de variedades sub-Riemannianas que possuem pmr produzidos pelo teorema 4.4 possuem distribuição geradora por colchetes. Precisaremos de uma maneira de determinar quando uma distribuição $G$-invariante $\mathcal{D}$ em $G / K$ é geradora por colchetes, através do subespaço $\mathcal{D}_{1 K} \subset \mathfrak{g} / \mathfrak{k}$. Começamos olhando mais de perto a definição de distribuição geradora por colchetes.

Vamos introduzir alguma terminologia. Se $\mathfrak{g}$ é uma álgebra de Lie (possivelmente de dimensão infinita) e se $\mathfrak{s} \subset \mathfrak{g}$ é um subespaço então definimos indutivamente uma seqüência $\mathfrak{s}^{i}$ de subespaços de $\mathfrak{g}$ fazendo $\mathfrak{s}^{1}=\mathfrak{s}$ e, supondo $\mathfrak{s}^{i}$ definido, tomamos $\mathfrak{s}^{i+1}$ como sendo o subespaço gerado por $\mathfrak{s}^{i}$ e pelos colchetes $[X, Y] \operatorname{com} X \in \mathfrak{s}^{i}$ e $Y \in \mathfrak{s}$. É claro que a seqüência $\left(\mathfrak{s}_{i}\right)_{i \in \mathbb{N}}$ de subespaços de $\mathfrak{g}$ é crescente.

Temos que $[X, Y] \in \mathfrak{s}^{i+j}$ quando $X \in \mathfrak{s}^{i}$ e $Y \in \mathfrak{s}^{j}$, ou abreviadamente, $\left[\mathfrak{s}^{i}, \mathfrak{s}^{j}\right] \subset \mathfrak{s}^{i+j}$. Isso segue facilmente por indução usando a identidade de Jacobi (mostra-se por indução em $i$ que $\left[\mathfrak{s}^{i}, \mathfrak{s}^{j}\right] \subset \mathfrak{s}^{i+j}$ para todo $j \in \mathbb{N}$ ). Denotamos por [s] a subálgebra de Lie de $\mathfrak{g}$ gerada por $\mathfrak{s}$. Segue então que $[\mathfrak{s}]=\bigcup_{i \in \mathbb{N}} \mathfrak{s}^{i}$.

Seja $M$ uma variedade. Denotamos por $\Gamma(T M)$ a álgebra de Lie dos campos vetoriais em $M$. Se $\mathcal{D} \subset T M$ é um subconjunto tal que $\mathcal{D}_{m}=\mathcal{D} \cap T_{m} M$ é um subespaço de $T_{m} M$ para todo $m \in M$ então denotamos por $\Gamma(\mathcal{D})$ o subespaço de $\Gamma(T M)$ formado pelos campos vetoriais em $M$ cuja imagem está contida em $\mathcal{D}$. Denotamos por $C^{\infty}(M)$ o anel de funções $f: M \rightarrow \mathbb{R}$.

Observação 4.4. Temos que $\Gamma(T M)$ é um módulo sobre $C^{\infty}(M)$, mas o colchete de Lie não é bilinear sobre $C^{\infty}(M)$, donde sempre consideramos $\Gamma(T M)$ como álgebra de Lie sobre $\mathbb{R}$. Recorde que o colchete de Lie $[X, Y]$ coincide com a derivada de Lie $\mathbb{L}_{X} Y$ e que a derivada de Lie $\mathbb{L}_{X}$ satisfaz a regra de Leibniz sobre derivação de um produto. Daí $[X, f Y]=X(f) Y+f[X, Y]$, para $f \in C^{\infty}(M)$ e $X, Y \in \Gamma(T M)$. Mais geralmente, temos:

$$
[f X, g Y]=f X(g) Y-g Y(f) X+f g[X, Y]
$$

para $f, g \in C^{\infty}(M), X, Y \in \Gamma(T M)$.

Seja agora $\mathcal{D}$ uma distribuição em $M$. Temos um subespaço $\Gamma(\mathcal{D})$ da álgebra de Lie $\Gamma(T M)$ e podemos considerar então a seqüência crescente $\left(\Gamma(\mathcal{D})^{i}\right)_{i \in \mathbb{N}}$ de subespaços de $\Gamma(T M)$. Para cada $m \in M$ e cada $i \in \mathbb{N}$ denotamos por $\mathcal{D}_{m}^{i}$ a imagem da aplicação 
$X \in \Gamma(\mathcal{D})^{i} \mapsto X_{m}$. Seja $\mathcal{D}^{i}=\bigcup_{m \in M} \mathcal{D}_{m}^{i}$. É claro que $\Gamma(\mathcal{D})^{i} \subset \Gamma\left(\mathcal{D}^{i}\right)$, mas em geral temos uma inclusão própria. É claro também que $\mathcal{D}^{i} \subset \mathcal{D}^{i+1}$ para $i \in \mathbb{N}$. Note que $\mathcal{D}^{1}=\mathcal{D}$, mas em geral $\mathcal{D}^{i}$ não é uma distribuição em $M$ para $i>1$ (a dimensão de $\mathcal{D}_{m}^{i}$ em geral depende de $m \in M$. Vide por exemplo a observação 4.5).

Temos que $\mathcal{D}$ é geradora por colchetes (vide observação 1.1) se e somente se a união $\bigcup_{i \in \mathbb{N}} \mathcal{D}_{m}^{i}$ coincide com $T_{m} M$ para todo $m \in M$. Como $T_{m} M$ tem dimensão finita, isso equivale a dizer que para todo $m \in M$ existe $i \in \mathbb{N}$ tal que $\mathcal{D}_{m}^{i}=T_{m} M$ (em geral, o valor de $i \in \mathbb{N}$ não pode ser escolhido independentemente de $m \in M)$. A seqüência $\left(\operatorname{dim} \mathcal{D}_{m}^{i}\right)_{i \in \mathbb{N}}$ é chamada o vetor de crescimento da distribuição $\mathcal{D}$ no ponto $m \in M$. A distribuição $\mathcal{D}$ é geradora por colchetes se e somente se para todo $m \in M$ o vetor de crescimento eventualmente atinge a dimensão de $M$.

O lema a seguir (mais precisamente, sua técnica de demonstração e seu corolário 1) nos mostram como calcular na prática os subespaços $\mathcal{D}_{m}^{i}$ de $T_{m} M$.

Lema 4.6. Seja $m \in M$. Então existe uma vizinhança aberta $U \subset M$ de $m$ tal que dado $i \in \mathbb{N}$ existem campos $X^{1}, \ldots, X^{r} \in \Gamma(\mathcal{D})^{i}$ tais que qualquer outro campo $X \in \Gamma(\mathcal{D})^{i}$ é tal que sua restrição $X_{\mid U}$ se escreve como combinação linear de $X_{\mid U}^{1}, \ldots, X_{\mid U}^{r}$ com coeficientes em $C^{\infty}(U)$.

Demonstração. Seja $m \in M$ e seja $U \subset M$ uma vizinhança aberta de $m$ tal que existem campos $Y^{1}, \ldots, Y^{k} \in \Gamma(\mathcal{D})$ tais que $Y_{\mid U}^{1}, \ldots, Y_{\mid U}^{k}$ é um referencial de $\mathcal{D}$ sobre $U$. Mostramos o resultado por indução em $i$. Para $i=1$ simplesmente tomamos $r=k$ e $X^{j}=Y^{j}$ para $j=1, \ldots, r$.

Sejam $X^{1}, \ldots, X^{r} \in \Gamma(\mathcal{D})^{i}$ tais que todo $X \in \Gamma(\mathcal{D})^{i}$ é tal que $X_{\mid U}$ é combinação linear dos campos $X_{\mid U}^{j}$ com coeficientes em $C^{\infty}(U)$. Daí os campos $X^{j}, Y^{l}$ e $\left[X^{j}, Y^{l}\right]$ para $j=1, \ldots, r, l=1, \ldots, k$ estão em $\Gamma(\mathcal{D})^{i+1}$ e todo $X \in \Gamma(\mathcal{D})^{i+1}$ é tal que $X_{\mid U}$ se escreve como combinação linear (das restrições a $U$ ) de tais campos com coeficientes em $C^{\infty}(U)$, o que segue facilmente de (4.1) (vide observação 4.4).

Corolário 1. Seja $m \in M$. Então existe uma vizinhança aberta $U \subset M$ de $m$ tal que dado $i \in \mathbb{N}$ existem campos $X^{1}, \ldots, X^{r} \in \Gamma(\mathcal{D})^{i}$ tais que os vetores $X_{x}^{1}, \ldots, X_{x}^{r}$ geram $\mathcal{D}_{x}^{i}$ para todo $x \in U$.

Demonstração. Basta escolher o aberto $U$ e os campos $X^{j}$ como no lema.

Corolário 2. Seja $i \in \mathbb{N}$. Se a dimensão de $\mathcal{D}_{m}^{i}$ não depende de $m \in M$ então $\mathcal{D}^{i}$ é uma distribuição (diferenciável) e numa vizinhança aberta de cada $m \in M$ existe um referencial de $\mathcal{D}^{i}$ formado por (restrições de) elementos de $\Gamma(\mathcal{D})^{i}$.

Demonstração. Seja $i \in \mathbb{N}$. Seja $m \in M$. Pelo corolário 1, podemos encontrar uma vizinhança aberta $U \subset M$ de $m$ e $X^{1}, \ldots, X^{r} \in \Gamma(\mathcal{D})^{i}$, tais que $X_{x}^{1}, \ldots, X_{x}^{r}$ geram $\mathcal{D}_{x}^{i}$ para todo $x \in U$. Reordenando, se necessário, podemos supor que $X_{m}^{1}, \ldots, X_{m}^{s}$ é uma base de $\mathcal{D}_{m}^{i}$ para algum $s \leq r$. Por continuidade, os vetores $X_{x}^{1}, \ldots, X_{x}^{s}$ são ainda linearmente independentes para $x$ numa vizinhança de $m$ e como $\mathcal{D}_{x}^{i}$ tem dimensão constante, segue que os mesmos formam uma base de $\mathcal{D}_{x}^{i}$. 
Corolário 3. Seja $i \in \mathbb{N}$. Se a dimensão de $\mathcal{D}_{m}^{i}$ não depende de $m \in M$ então dados campos vetoriais $X, Y$ num aberto $V$ de $M$ tais que $X$ toma valores em $\mathcal{D}^{i}$ e $Y$ toma valores em $\mathcal{D}$, segue que $[X, Y]$ toma valores em $\mathcal{D}^{i+1}$.

Demonstração. Seja $m \in V$. Pelo corolário 2, podemos encontrar $X^{1}, \ldots, X^{r} \in \Gamma(\mathcal{D})^{i}$ e $Y^{1}, \ldots, Y^{k} \in \Gamma(\mathcal{D})$ tais que as restrições dos campos $X^{j}$ a uma vizinhança aberta $U \subset V$ de $m$ formam um referencial de $\mathcal{D}^{i}$ e as restrições a $U$ dos campos $Y^{l}$ formam um referencial de $\mathcal{D}$. Observe que o campo $\left[X^{j}, Y^{l}\right] \in \Gamma(\mathcal{D})^{i+1}$ toma valores em $\mathcal{D}^{i+1}$. Os campos $X_{\mid U}$ e $Y_{\mid U}$ se escrevem como combinação linear dos campos $X_{\mid U}^{j}$ e $Y_{\mid U}^{l}$, respectivamente, com coeficientes em $C^{\infty}(U)$. O resultado segue de (4.1) (vide observação 4.4).

Observação 4.5. O corolário acima não é válido sem a hipótese que $\mathcal{D}_{m}^{i}$ tenha dimensão constante. Por exemplo, seja $M=\mathbb{R}^{3}$ e seja $\mathcal{D}$ a distribuição de posto 2 gerada pelos campos $\frac{\partial}{\partial y}$ e $\frac{\partial}{\partial x}+y^{3} \frac{\partial}{\partial z}$. Daí, para $m=(x, y, z)$, temos que $\mathcal{D}_{m}^{2}=\mathbb{R}^{3}$ quando $y \neq 0$ e $\mathcal{D}_{m}^{2}$ é o plano gerado por $\frac{\partial}{\partial x}$ e $\frac{\partial}{\partial y}$ quando $y=0$. Também $\mathcal{D}^{3}=\mathcal{D}^{2}$ e $\mathcal{D}^{4}=T \mathbb{R}^{3}$. Note que o campo $y \frac{\partial}{\partial z}$ toma valores em $\mathcal{D}^{2}$ e o campo $\frac{\partial}{\partial y}$ toma valores em $\mathcal{D}$, mas o colchete de Lie $\left[\frac{\partial}{\partial y}, y \frac{\partial}{\partial z}\right]=\frac{\partial}{\partial z}$ não toma valores em $\mathcal{D}^{3}$.

Relacionamos agora o colchete de Lie em $\mathfrak{g}$ com o colchete de Lie de certos campos vetoriais na variedade $G / K$.

Observação 4.6. Se $\mathfrak{m}$ é um subespaço complementar de $\mathfrak{k}$ em $\mathfrak{g}$ então sempre existem seções locais $s: U \subset G / K \rightarrow G \operatorname{com} 1 K \in U, s(1 K)=1$ e tal que a imagem de $d s_{1 K}$ seja $\mathrm{m}$. Podemos, por exemplo, considerar a inversa da restrição de $\pi$ à imagem pela exponencial de uma vizinhança aberta suficientemente pequena de $0 \mathrm{em} \mathfrak{m}$.

Lema 4.7. Sejam $G$ um grupo de Lie e $K \subset G$ um subgrupo fechado, com álgebras de Lie $\mathfrak{g}$ e $\mathfrak{k}$ respectivamente. Seja $\mathfrak{m} \subset \mathfrak{g}$ um subespaço complementar de $\mathfrak{k}$ em $\mathfrak{g}$ e seja $s: U \subset G / K \rightarrow G$ uma seção local tal que $1 K \in U, s(1 K)=1$ e tal que a imagem de $d s_{1 K}$ seja m. Para $X \in \mathfrak{g}$ defina um campo vetorial $\vec{X}$ em $U$ fazendo $\vec{X}(g K)=s(g K) \bar{\pi}(X)$. Daí, para $X, Y \in \mathfrak{m}$ temos $[\vec{X}, \vec{Y}]_{1 K}={\overrightarrow{[X, Y]_{1 K}}}_{\text {. }}$.

Demonstração. Seja $g K \in U$ e suponha $g=s(g K)$. Diferenciando a identidade $\pi \circ l_{g}=\bar{l}_{g} \circ \pi$ no ponto 1 e aplicando sobre um vetor $X \in \mathfrak{g}$ concluímos que:

$$
\vec{X}_{g K}=d \pi_{g}\left(X_{g}^{L}\right)
$$

onde $X^{L}$ é o campo vetorial invariante à esquerda tal que $X_{1}^{L}=X$.

Seja $f: U \rightarrow \mathbb{R}$ uma função. Basta mostrar que $[\vec{X}, \vec{Y}]_{1 K}$ e $\overrightarrow{[X, Y]_{1 K}}=\bar{\pi}([X, Y])$ coincidem quando aplicados sobre $f$ (como derivadas direcionais). Segue de (4.7.1) que:

$$
\vec{X}(f)=X^{L}(f \circ \pi) \circ s .
$$

Como $\bar{\pi} \circ d s_{1 K}=$ Id e $Y \in \mathfrak{m}$ temos $d s_{1 K} \circ \bar{\pi}(Y)=Y$. Aplicando o vetor $\vec{Y}_{1 K}=\bar{\pi}(Y)$ nos dois lados de (4.7.2) vem:

$$
\vec{Y} \vec{X}(f)_{1 K}=Y^{L} X^{L}(f \circ \pi)_{1}
$$


Trocando os papéis de $X$ e $Y$ em (4.7.3) e subtraindo as duas equações obtidas segue o resultado desejado.

Seja $f: M \rightarrow M$ um difeomorfismo. Para $X \in \Gamma(T M)$ definimos o pull-back $f^{*} X$ de $X$ por $f$ fazendo $\left(f^{*} X\right)_{m}=d f_{m}^{-1}\left(X_{f(m)}\right)$. Temos que $f^{*}$ é um automorfismo da álgebra de Lie $\Gamma(T M)$. Se $\mathcal{D}$ é uma distribuição em $M$ e $f$ preserva $\mathcal{D}$ então $f^{*}$ deixa o subespaço $\Gamma(\mathcal{D})$ invariante. Segue que $f^{*}$ deixa $\Gamma(\mathcal{D})^{i}$ invariante para todo $i \in \mathbb{N}$. Segue daí que $\mathcal{D}_{f(m)}^{i}=d f_{m}\left(\mathcal{D}_{m}^{i}\right)$ para todo $m \in M$ e todo $i \in \mathbb{N}$.

Se temos um grupo de Lie $G$ agindo transitivamente em $M$ e se $\mathcal{D}$ é $G$-invariante então $\mathcal{D}^{i}$ é uma distribuição $G$-invariante para todo $i \in \mathbb{N}$ (segue do corolário 2 do lema 4.6 que $\mathcal{D}^{i}$ é de fato uma distribuição).

Considere agora uma distribuição $G$-invariante $\mathcal{D}$ em $M=G / K$, onde $G$ é um grupo de Lie e $K \subset G$ é um subgrupo fechado. Seja m um subespaço complementar de $\mathfrak{k}$ em $\mathfrak{g}$ e seja $\mathfrak{d}$ o subespaço de $\mathfrak{m}$ tal que $\bar{\pi}(\mathfrak{d})=\mathcal{D}_{1 K}$. Segue por indução, usando a identidade de Jacobi, que $\left[\mathfrak{k}, \mathfrak{d}^{i}\right] \subset \mathfrak{k}+\mathfrak{d}^{i}$ (o caso $i=1$ segue da $G$-invariância de $\mathcal{D}$. Vide observação 2.2). Segue agora, por indução também, que $(\mathfrak{k}+\mathfrak{d})^{i}=\mathfrak{k}+\mathfrak{d}^{i}$. Em particular, $[\mathfrak{k}+\mathfrak{d}]=\mathfrak{k}+[\mathfrak{d}]$. Temos finalmente o seguinte teorema.

Teorema 4.8. Sejam $G$ um grupo de Lie e $K \subset G$ um subgrupo fechado, com álgebras de Lie $\mathfrak{g}$ e $\mathfrak{k}$ respectivamente. Seja $\mathfrak{m} \subset \mathfrak{g}$ um subespaço complementar de $\mathfrak{k}$ em $\mathfrak{g}$ e seja $\mathcal{D}$ uma distribuição $G$-invariante em $G / K$. Seja $\mathfrak{d} \subset \mathfrak{m}$ o subespaço tal que $\bar{\pi}(\mathfrak{d})=\mathcal{D}_{1 K}$. Então $\bar{\pi}\left(\mathfrak{d}^{i}\right)=\bar{\pi}\left((\mathfrak{k}+\mathfrak{d})^{i}\right)=\mathcal{D}_{1 K}^{i}$ para todo $i \in \mathbb{N}$.

Demonstração. Seja $s: U \subset G / K \rightarrow G$ uma seção local tal que $1 K \in U, s(1 K)=1$ e tal que a imagem de $d s_{1 K}$ seja $\mathfrak{m}$ (vide observação 4.6). Para $X \in \mathfrak{g}$ definimos $\vec{X}$ como no enunciado do lema 4.7 .

Mostramos o resultado por indução em $i$. O caso $i=1$ é trivial, já que $\mathfrak{d}^{1}=\mathfrak{d}$ e $\mathcal{D}^{1}=\mathcal{D}$. Suponha que $\bar{\pi}\left(\mathfrak{d}^{i}\right)=\mathcal{D}_{1 K}^{i}$.

Mostremos primeiramente que $\bar{\pi}\left(\mathcal{D}^{i+1}\right) \subset \mathcal{D}_{1 K}^{i+1}$. Basta ver que $\bar{\pi}([X, Y]) \in \mathcal{D}_{1 K}^{i+1}$ sempre que $X \in \mathfrak{d}^{i}$ e $Y \in \mathfrak{d}$. Escreva $X=X_{1}+X_{2} \operatorname{com} X_{1} \in \mathfrak{k}$ e $X_{2} \in \mathfrak{m}$. Temos:

$$
\bar{\pi}([X, Y])=\bar{\pi}\left(\left[X_{1}, Y\right]\right)+\bar{\pi}\left(\left[X_{2}, Y\right]\right)
$$

onde o primeiro termo pertence a $\bar{\pi}(\mathfrak{d})=\mathcal{D}_{1 K}$. Resta ver que $\bar{\pi}\left(\left[X_{2}, Y\right]\right) \in \mathcal{D}_{1 K}^{i+1}$. Como $X_{2}, Y \in \mathfrak{m}$, podemos usar o lema 4.7 e obtemos:

$$
\bar{\pi}\left(\left[X_{2}, Y\right]\right)={\overrightarrow{\left[X_{2}, Y\right]_{1 K}}}_{1}=\left[\vec{X}_{2}, \vec{Y}\right]_{1 K}
$$

Mas $\vec{X}_{2}$ toma valores em $\mathcal{D}^{i}$ e $\vec{Y}$ toma valores em $\mathcal{D}$, donde pelo corolário 3 do lema 4.6, vemos que $\left[\vec{X}_{2}, \vec{Y}\right]$ toma valores em $\mathcal{D}^{i+1}$.

Resta mostrar que $\mathcal{D}_{1 K}^{i+1} \subset \bar{\pi}\left(\mathcal{d}^{i+1}\right)$. Seja $\mathfrak{d}^{\prime} \subset \mathrm{m}$ o subespaço tal que $\bar{\pi}\left(\mathfrak{d}^{\prime}\right)=\mathcal{D}_{1 K}^{i}$. Seja $X^{1}, \ldots, X^{r}$ uma base de $d^{\prime}$ e seja $Y^{1}, \ldots, Y^{k}$ uma base de $\mathfrak{d}$. Daí os campos $\vec{X}^{j}, j=1, \ldots, r$ formam um referencial de $\mathcal{D}^{i}$ e os campos $\vec{Y}^{l}, l=1, \ldots, k$ formam um referencial de $\mathcal{D}$. Pelo lema 4.7 temos:

$$
\bar{\pi}\left(\left[X^{j}, Y^{l}\right]\right)=\overrightarrow{\left[X^{j}, Y^{l}\right]_{1 K}}=\left[\vec{X}^{j}, \vec{Y}^{l}\right]_{1 K}
$$


para $j=1, \ldots, r$ e $l=1, \ldots, k$. Note que $X^{j} \in \mathfrak{k}+\mathfrak{d}^{i}$, donde $\left[X^{j}, Y^{l}\right] \in \mathfrak{k}+\mathfrak{d}^{i+1}$. Concluímos que $\left[\vec{X}^{j}, \vec{Y}^{l}\right]_{1 K} \in \bar{\pi}\left(\mathfrak{d}^{i+1}\right)$.

Segue agora de (4.1) (vide observação 4.4) que se $X \in \Gamma\left(\mathcal{D}^{i}\right), Y \in \Gamma(\mathcal{D})$ então $[X, Y]_{1 K} \in \bar{\pi}\left(\mathfrak{d}^{i+1}\right)$. Mas $\Gamma(\mathcal{D})^{i+1}$ é gerado por $\Gamma(\mathcal{D})^{i}$ e por colchetes de elementos de $\Gamma(\mathcal{D})^{i}$ por elementos de $\Gamma(\mathcal{D})$. Como obviamente $\Gamma(\mathcal{D})^{i} \subset \Gamma\left(\mathcal{D}^{i}\right)$ segue que $Z_{1 K} \in \bar{\pi}\left(\mathfrak{d}^{i+1}\right)$ sempre que $Z \in \Gamma(\mathcal{D})^{i+1}$. O resultado segue.

Corolário. Nas condições do teorema, temos que $\mathcal{D}$ é geradora por colchetes se e somente se $\mathfrak{k}+\mathfrak{d}=\bar{\pi}^{-1}\left(\mathcal{D}_{1 K}\right)$ gera $\mathfrak{g}$ como álgebra de Lie, i.e., se e somente se $\mathfrak{k}+[\mathfrak{d}]=[\mathfrak{k}+\mathfrak{d}]=\mathfrak{g}$.

Demonstração. Segue diretamente do teorema.

Aplicamos agora o corolário acima para as variedades que aparecem no teroema 4.4. Temos o seguinte.

Teorema 4.9. Nas condições do teorema 4.4, temos que $\mathcal{D}$ é uma distribuição geradora por colchetes.

Demonstração. Podemos identificar $M \operatorname{com} G / K$ da maneira usual e daí o corolário do teorema 4.8 nos diz que basta mostrar que $\mathfrak{k}+\mathfrak{d}$ gera $\mathfrak{g}$ como álgebra de Lie. Observe que a subálgebra de Lie complexa de $\mathfrak{g}^{c}$ gerada por um subespaço real $\mathfrak{s} \subset \mathfrak{g}$ coincide com a complexificação da subálgebra de Lie real gerada por $\mathfrak{s}$ em $\mathfrak{g}$.

Basta mostrar então que $\mathfrak{k}+\mathfrak{d}$ gera $\mathfrak{g}^{c}$ como subálgebra de Lie complexa. Resta então ver que tal subálgebra gerada contém a. Mas para $\alpha \in \Delta$ temos $\mathfrak{g}_{\alpha}^{c} \subset \mathfrak{k}^{c}+\mathfrak{d}^{c}$ (vide decomposição (4.4.5)) e fazendo colchetes de Lie de elementos de $\mathfrak{g}_{\alpha}^{c}$ com elementos de $\mathfrak{g}_{-\alpha}^{c}$ obtemos $\mathbb{C} H_{\alpha}$ (vide observação 3.2.1). Os vetores $H_{\alpha}$ geram $\mathfrak{h}^{c}$ e $\mathfrak{a}^{c} \subset \mathfrak{h}^{c}$.

Analisamos agora sob que condições a distribuição que aparece no teorema 4.4 é fortemente geradora por colchetes (ou de contato). Observe que quando a distribuição é fortemente geradora por colchetes, a variedade sub-Riemmanniana trivialmente possui pmr (vide corolário do lema 1.4). Como mostraremos a seguir, isso em geral não ocorre nos exemplos produzidos pelo teorema 4.4.

Teorema 4.10. Nas condições do teorema 4.4, temos que $\mathcal{D}$ é de contato quando a tem dimensão 1 e quando $\operatorname{dim} \mathfrak{a}>1$ a distribuição $\mathcal{D}$ não é fortemente geradora por colchetes.

Demonstração. Observando que a dimensão de a é exatamente a codimensão de $\mathcal{D}$ (na demonstração do teorema 4.4 foi observado que $\mathfrak{g}=\mathfrak{k} \oplus \mathfrak{d} \oplus \mathfrak{a}$ ) e recordando que quando $\mathcal{D}$ tem codimensão 1 então $\mathcal{D}$ é de contato se e somente se $\mathcal{D}$ é fortemente geradora por colchetes, vemos que o teorema se reduz a mostrar que $\mathcal{D}$ é fortemente geradora por colchetes se e somente se a tem dimensão 1 .

Utilizamos o lema 1.4. Devemos então estudar o núcleo de $\omega_{\mid \mathcal{D}^{0}}$ fora da seção nula de $\mathcal{D}^{0}$. Utilizamos então o teorema 2.1 e fixamos $\mathfrak{m}$ igual ao complemento $\mathcal{K}$-ortogonal de $\mathfrak{k}$ em $\mathfrak{g}$ (que é de fato um subespaço complementar de $\mathfrak{k}$, como já foi observado na demonstração do teorema 4.4). Temos então que um vetor $(v, \zeta) \in T_{g K}(G / K) \times \mathfrak{d}^{0}$ pertence ao núcleo de $\left(f^{*} \omega\right)_{\mid U \times \mathcal{o}^{\circ}}$ no ponto $(g K, \lambda) \in U \times \mathfrak{d}^{0}$ se e somente se:

$$
w \in \mathfrak{d} \quad \text { e } \quad \zeta=\left(\pi_{\mathrm{m}} \circ \operatorname{ad}_{\hat{v}} \circ i_{\mathfrak{m}}\right)^{*} \lambda,
$$

$\operatorname{com} \hat{v}=s(g K)^{-1} d s_{g K}(v), w=\pi_{\mathrm{m}}(\hat{v})=s(g K)^{-1} v$ (vide observação 2.4). Repetimos agora alguns argumentos da demonstração do teorema 4.4 . 
Podemos identificar $\mathfrak{m}$ com $\mathrm{m}^{*}$ através do isomorfismo $X \mapsto \mathcal{K}(X, \cdot)$ e daí $\mathfrak{d}^{0}$ fica identificado com a. Como $\pi_{\mathfrak{m}} \circ \operatorname{ad}_{\hat{v}} \circ i_{\mathfrak{m}}$ é $\mathcal{K}$-antisimétrica vemos que (4.10.1) equivale a:

$$
w \in \mathfrak{d} \quad \text { e } \quad \zeta=-\pi_{\mathfrak{m}}([\hat{v}, \lambda]) .
$$

Como $\hat{v}-w \in \mathfrak{k}$ e $\lambda \in \mathfrak{a}$, vemos que podemos trocar $\hat{v}$ por $w$ em (4.10.2). Mas $w \in \mathfrak{o}$ $\mathrm{e}[\mathfrak{a}, \mathfrak{d}] \subset \mathfrak{d} \subset \mathfrak{m}$, donde a projeção $\pi_{\mathfrak{m}}$ pode ser desprezada. Daí (4.10.2) equivale a:

$$
w \in \mathfrak{d} \quad \text { e } \quad \zeta=-[w, \lambda]
$$

$\operatorname{Mas}[w, \lambda] \in \mathfrak{o}$ e $\zeta \in \mathfrak{a}$, donde $\zeta=[w, \lambda]=0$. Segue que o núcleo de $\left(f^{*} \omega\right)_{\mid U \times \mathfrak{o}^{0}}$ no ponto $(g K, \lambda)$ (ou o núcleo de $\omega_{\mid \mathcal{D}^{\circ}}$ no ponto $f(g K, \lambda)$ ) é trivial se e somente se $\mathfrak{z}(\lambda) \cap \mathfrak{d}=0$, i.e., $\lambda$ não comuta com elementos não nulos de $\mathfrak{o}$.

Segue que $\mathcal{D}$ é fortemente geradora por colchetes se e somente se $\mathfrak{z}(\lambda) \cap \mathfrak{d}=0$ para todo $\lambda \in \mathfrak{a}, \lambda \neq 0$. Observe que $\mathfrak{z}(\lambda) \cap \mathfrak{d}=0$ é equivalente a $\mathfrak{z}_{\mathfrak{g}^{c}}(\lambda) \cap \mathfrak{d}^{c}=0$ e que $\mathfrak{z}_{\mathfrak{g}^{c}}(\lambda)$ é dado como em (4.4.4). Utilizando a decomposição (4.4.5) vemos que $z(\lambda) \cap \mathfrak{d}=0$ se e somente se não existe $\alpha \in \Delta \operatorname{com} \alpha_{\mid \mathfrak{a}} \neq 0$ e $\alpha(\lambda)=0$.

Concluímos que $\mathcal{D}$ é fortemente geradora por colchetes se e somente se para todo $\alpha \in \Delta$ temos $\alpha_{\mid \mathfrak{a}}=0$ ou $\operatorname{Ker} \alpha_{\mid \mathfrak{a}}=0$. Como a imagem de $\alpha_{\mid \mathfrak{a}}$ tem dimensão (real) menor ou igual a 1 (vide corolário do lema 3.3.8), segue que isso ocorre se e somente se $\operatorname{dim} \mathfrak{a}=1$ (não é possível que $\alpha_{\mid \mathfrak{a}}=0$ para todo $\alpha \in \Delta$, pois isso implicaria que a está contido no centro de $\mathfrak{g}$ ). 


\section{Bibliografia.}

[B] C. Bär. Carnot Caratheodory Metriken. Diplomarbeit, Bonn, 1988.

[Fe] P. J. Fernandez. Medida e Integração. Rio de Janeiro, Instituto de Matemática Pura e Aplicada, CNPq, 1976 (projeto Euclides).

[FG] E. Falbel e C. Gorodski. Sub-Riemannian homogeneous spaces in dimensions 3 and 4. Geometriae Dedicata, 1996.

[GK] C. Golé e R. Karidi. A note on Carnot geodesics in nilpotent Lie groups. J. Dyn. and Control Syst., Vol. 1, No. 4, 1995, 535-549.

[Hm] U. Hamenstädt. Some regularity theorems for Carnot-Carathéodory metrics. J. of Diff. Geometry 32 (1990), pp. 819-850.

[KN] S. Kobayashi e K. Nomizu. Foundations of differential geometry. Vol. 1. New York, Interscience, 1963.

[Kp] I. Kupka. Géométrie sous-Riemanniene. In Séminaire Bourbaki, número 817, 1995-96.

[Kp2] I. Kupka. Abnormal extremals, preprint, 1992.

[LS] W. Liu e H. J. Sussmann. Shortest Paths for Sub-Riemannian Metrics on Rank-2 Distributions. Memoirs of the AMS. Number 564, Vol. 118, November, 1995.

[Mg] R. Montgomery. A survey of singular curves in sub-Riemannian geometry. J. Dyn. and Control Syst., 1:1:49-90, 1995.

[Mg2] R. Montgomery. Abnormal minimizers, to appear in SIAM J. Control and Opt.

[Mg3] R. Montgomery. Singular extremals on Lie groups. Math. Control Signals Systems (1994) $7: 217-234$.

[Sm] H. J. Sussmann. A weak regularity theorem for real analytic optimal control problems. Rev. Mat. Iberoamer., 2(3)(1986), 307-317.

[St] R. Strichartz. Sub-Riemannian geometry. J. Diff. Geometry 24 (1986), pp. 221-263.

[VG] A. M. Vershik e V. Ya. Gershkovich. Non-holonomic Dynamical Systems, Geometry of Distributions and Variational Problems, volume 16 da Encyclopaedia of Mathematical Sciences series, páginas 1-81. Springer-Verlag, 1994. Original russo 1987.

[Vj] V. S. Varadarajan. Lie groups, Lie algebras and their representations. Prentice-Hall series in modern analysis, 1974, New Jersey.

[W] J. A. Wolf. Spaces of constant curvature. New York, Mc-Graw-Hill, 1967.

[Wn] F. W. Warner. Foundations of differentiable manifolds and Lie groups. Glenview, Scott, 1971. 